\title{
Noncoherent Physical-Layer Network Coding with Frequency-Shift Keying Modulation
}

\author{
Terry Ferrett
}

Follow this and additional works at: https://researchrepository.wvu.edu/etd

\section{Recommended Citation}

Ferrett, Terry, "Noncoherent Physical-Layer Network Coding with Frequency-Shift Keying Modulation" (2017). Graduate Theses, Dissertations, and Problem Reports. 5592.

https://researchrepository.wvu.edu/etd/5592

This Dissertation is protected by copyright and/or related rights. It has been brought to you by the The Research Repository @ WVU with permission from the rights-holder(s). You are free to use this Dissertation in any way that is permitted by the copyright and related rights legislation that applies to your use. For other uses you must obtain permission from the rights-holder(s) directly, unless additional rights are indicated by a Creative Commons license in the record and/ or on the work itself. This Dissertation has been accepted for inclusion in WVU Graduate Theses, Dissertations, and Problem Reports collection by an authorized administrator of The Research Repository @ WVU.

For more information, please contact researchrepository@mail.wvu.edu. 


\title{
Noncoherent Physical-Layer Network Coding with Frequency-Shift Keying Modulation
}

\author{
Terry Ferrett \\ Dissertation submitted to the \\ College of Engineering and Mineral Resources \\ at West Virginia University \\ in partial fulfillment of the requirements \\ for the degree of
}

Doctor of Philosophy

in

Electrical Engineering

Daryl S. Reynolds

Natalia A. Schmid

Vinod K. Kulathumani

Erdogan Gunel

Matthew C. Valenti, Chair

Lane Department of Computer Science and Electrical Engineering

Morgantown, West Virginia

2017

Keywords: Physical-Layer Network Coding, Noncoherent Detection, Frequency Shift

Keying,Channel Estimation, Channel Code Design, Two-way Relay Channel

Copyright 2017 Terry Ferrett 


\begin{abstract}
Noncoherent Physical-Layer Network Coding with Frequency-Shift Keying Modulation Terry Ferrett
\end{abstract}

The rapid growth of wireless communication technology has motivated novel approaches into improving performance. A major avenue of research investigates the benefit of relaying, where wireless devices outside radio range of each other communicate by passing information through a device in between. Traditionally, devices communicating through a relay transmit at separate times to avoid interfering with each other. Physical-layer network coding is a recent technique that improves throughput by allowing devices to transmit at the same time to the relay, deliberately interfering. This dissertation develops a system performing physicallayer network coding in the topology where two devices exchange information through a single relay. Many signaling techniques require synchronized carrier phases and frequencies for all three devices, which can be challenging to achieve in some scenarios. To alleviate the need for synchronization, this work develops a noncoherent system that requires only frame and symbol synchronization and relaxes the need for carrier synchronization. To combat the degrading effects of the wireless channel, the system utilizes bit-interleaved coded modulation (BICM) along with powerful iterative LDPC and turbo coding. The modulation considered, M-ary frequency-shift keying, is suitable for noncoherent reception and has constant envelope and high energy efficiency. Two formulations of demodulation are developed, one that requires knowledge of the fading amplitudes, and the other that requires only knowledge of the average power. The LDPC codes are optimized for the particular scheme by using extrinsic information transfer (EXIT) charts to identify promising variable-node degree distributions. Simulation results illustrate the efficacy of the proposed demodulator when it is combined with the optimized LDPC codes. The simulation results agree with the coded modulation (CM) capacities, which are also developed. Throughout this work, the capacity and error rate performance of the developed receiver is compared against conventional network coding where the end nodes avoid interfering by transmitting in different times or bands. 


\section{Acknowledgements}

I have been extremely fortunate to be surrounded by inspiring individuals who made this contribution possible. Dr. Matthew Valenti is a patient, supportive mentor and friend who recognized my passion for creative challenges. I would like to thank my committee for their unwavering dedication. Dr. Brian Woerner made tremendous efforts to ensure that I was supported while performing research. The past and present students of the Wireless Communications Research Lab at WVU provided endless encouragement. Most of all I would like to thank my parents, who sacrificed deeply for me and taught me the value of persistence.

My results could not have been produced without support from several generous sources. Various stages of my assistantship were supported by National Science Foundation (NSF) Award No. CNS-0750821 and Army Research Laboratory Contract W911NF-10-0109. Construction of the computing cluster used to generate my simulation results was funded by CNS-0750821. Support for design and implementation of software to enable biometrics researchers to execute algorithms on the cluster was provided by National Science Foundation Awards No. I/UCRC FRP 1332118 and 1066197. Thanks to support from Dr. Hideki Ochiai and NSF Award No. EAPSI-1107543, I spent a summer at Yokohama National University in Ochiai Lab to further my research.

I had the great fortune to work alongside student workers and staff in the WVU LCSEE Systems support group. In my role as a system administrator I learned fundamental principles for designing and supporting GNU/Linux deployments from David Krovich. As a helpdesk support technician I was mentored by Marc Seery, who was instrumental in retaining me as a technician. I would like to specially thank Henry Graham for opening his ambitions to system administration and graciously allowing me to tutor him at the start of his career. 


\section{Contents}

$\begin{array}{ll}\text { Acknowledgements } & \text { iii }\end{array}$

List of Figures vii

List of Tables $\quad$ x

Notation $\quad$ xi

1 Introduction $\quad 1$

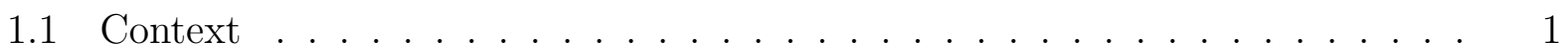

1.1.1 Physical Layer Network Coding . . . . . . . . . . . . . . . . 3

1.1.2 Noncoherent Frequency Shift Keying . . . . . . . . . . . . . . . . 5

1.1.3 Channel Coding . . . . . . . . . . . . . . . . . . 8

1.2 Summary of Contributions . . . . . . . . . . . . . . . . . . . . . . . . . . . . . . .

1.3 System Model Elements used Throughout . . . . . . . . . . . . . . . . . . . 10

1.3.1 Transmission by End Nodes . . . . . . . . . . . . . . . . . . 11

1.3.2 Channel Model for Multiple-Access Stage . . . . . . . . . . . . . . 11

1.4 Conclusion . . . . . . . . . . . . . . . . . . . . . . . 13

2 Noncoherent Binary FSK System for DNC 14

2.1 Introduction . . . . . . . . . . . . . . . . . . . . . . . . . . . . . . . . . . . .

2.2 System Model . . . . . . . . . . . . . . . . . . . . . . . . . . . . . . . . . . . . . . . . . . . . . . . .

2.3 Relay Receiver . . . . . . . . . . . . . . . . . . . . 17

2.3.1 Link-Layer Network Coding Receiver . . . . . . . . . . . . . . . . . . 18

2.3.2 Physical-Layer Network Coding Receiver . . . . . . . . . . . . . . . . 19

2.4 Channel Estimator . . . . . . . . . . . . . . . . . . . 25

2.4.1 Fading Amplitude Estimator . . . . . . . . . . . . . . . 25

2.4.2 Transmission-Case Detection . . . . . . . . . . . . . . . 27

2.4.3 Amplitude Estimation for Single-Transmitter Links . . . . . . . . . . 27

2.5 Simulation Study . . . . . . . . . . . . . . . . . . . . . . 28

2.5.1 Uncoded Performance with Perfect Channel Estimates . . . . . . . . 28

2.5.2 Uncoded Performance with Channel Estimation . . . . . . . . . . . . 31 
2.5.3 Performance with an Outer turbo Code . . . . . . . . . . . . . . . 32

2.6 Conclusion . . . . . . . . . . . . . . . . . . 35

3 Iterative Noncoherent M-ary FSK System for DNC 37

3.1 Introduction . . . . . . . . . . . . . . . . . . . . . 37

3.2 System Model . . . . . . . . . . . . . . . . . . . . . . . . 39

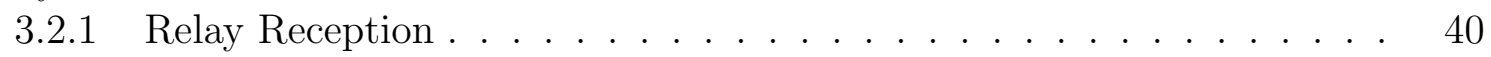

3.2 .2 Broadcast Phase . . . . . . . . . . . . . . . . . . . 41

3.3 Digital Network-Coded Relay Demodulator . . . . . . . . . . . . . . . . 42

3.3.1 Super-Symbol Probability Distributions . . . . . . . . . . . . 44

3.4 Capacity . . . . . . . . . . . . . . . . . . . . . 47

3.4.1 End-to-End Capacity Analysis . . . . . . . . . . . . . . . . . 47

3.4 .2 Capacity Analysis for Multiple-Access Phase . . . . . . . . . . . . . . 49

3.4.3 Simulated Capacity . . . . . . . . . . . . . . . . . 50

3.5 LDPC Coded Performance . . . . . . . . . . . . . . . . . 54

3.5.1 Bit Error Rate Simulation Procedure . . . . . . . . . . . . . . . 54

3.5.2 Channel-Coded Performance . . . . . . . . . . . . . 56

3.6 Conclusion . . . . . . . . . . . . . . . . . . . . . . 57

4 LDPC Code Design for DNC $\quad 59$

4.1 Introduction . . . . . . . . . . . . . . . . . . 59

4.2 LDPC Code Optimization . . . . . . . . . . . . . . . . 61

4.2.1 Optimization through Selection of Variable Node Degree . . . . . . . 63

4.3 EXIT-Optimized LDPC Code Performance . . . . . . . . . . . . . . . 65

4.3.1 Optimization Procedure . . . . . . . . . . . . . 65

4.3.2 Optimization Results . . . . . . . . . . . . . . . . . 67

4.4 Conclusion . . . . . . . . . . . . . . . . . . . 72

5 Iterative Noncoherent M-ary FSK System for ANC $\quad 75$

5.1 Introduction . . . . . . . . . . . . . . . . . . . . 75

5.2 System Model . . . . . . . . . . . . . . . . . . . . . . . . . . . . . . . . . . . . . . 77

5.2.1 Analog Network Coding at the Relay . . . . . . . . . . . . . . . 77

5.2 .2 End Node Reception . . . . . . . . . . . . . . . . . . . . . . 79

5.3 Noncoherent End Node Demodulator . . . . . . . . . . . . . . . . 80

5.3.1 End Node Received Symbol Distribution . . . . . . . . . . . . . . 80

5.3.2 Iterative Demodulation and Decoding . . . . . . . . . . . . . 83

5.4 Demodulator Performance . . . . . . . . . . . . . . . . . 85

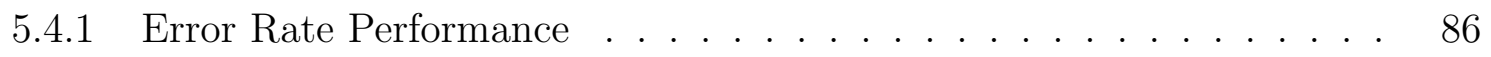

5.5 Conclusion . . . . . . . . . . . . . . . . . . . . 89 
6 Other Contributions $\quad 90$

6.1 Physical-layer Network Coding Using

FSK Modulation with Frequency Offset . . . . . . . . . . . . . . . . . 90

6.1 .1 Introduction . . . . . . . . . . . . . . . . . . . . . . . . . . . . . . . .

6.1.2 System Model . . . . . . . . . . . . . . . . . . . . . . . . . . . . . . . . . 92

6.1.3 Detection Rule . . . . . . . . . . . . . . . . . . 93

6.1.4 Simulation Results . . . . . . . . . . . . . . . . . . . 99

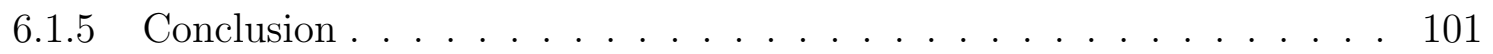

6.2 Reduced Complexity Detection for Network-Coded Slotted ALOHA Using

Sphere Decoding . . . . . . . . . . . . . . . . . . 103

6.2 .1 Introduction . . . . . . . . . . . . . . . . . . . 103

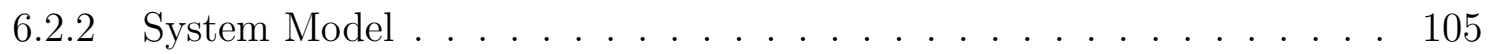

6.2 .3 List Sphere Decoder . . . . . . . . . . . . . . . . . . . . 108

6.2.4 Simulation Results . . . . . . . . . . . . . . . . . . . . . 112

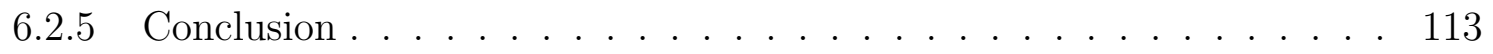

7 Future Work 115

7.1 Non-Orthogonal FSK

with Bandwidth Constraint . . . . . . . . . . . . . . . . 115

7.2 Analytical Performance Bounds . . . . . . . . . . . . . . . . . 116

7.3 Channel Code Construction . . . . . . . . . . . . . . . . . . 116

7.4 Improved Analog Network Coding . . . . . . . . . . . . . . . . . . 118

$\begin{array}{lr}\text { References } & 119\end{array}$ 


\section{List of Figures}

1.1 Examples of wireless communication. . . . . . . . . . . . . . . . .

1.2 Two-way relay channel (a) and schedules for several exchange techniques: All links modeled as point-to-point (b), link-layer network coding (c), physicallayer network coding $(\mathrm{d}) \ldots \ldots \ldots$. . . . . . . . . . . . . 3

1.3 Correlation-type detector for noncoherent binary frequency-shift keying. . . . 7

2.1 System Model for DNC two-way relay channel multiple-access stage. . . . . . 17

2.2 Bit error rate at the relay in Rayleigh fading when DNC and LNC is used and $\mathcal{E}_{2}=\mathcal{E}_{1}$. Depending on the amount of channel state information that is available, the PNC system will use one of three different relay receivers. . .

2.3 Bit error rate at the relay in Rayleigh fading when DNC is used with three different receivers and either $\mathcal{E}_{2}=\mathcal{E}_{1}$ (solid line) or $\mathcal{E}_{2}=4 \mathcal{E}_{1}$ (dashed line). .

2.4 Influence of fading-block length $N$ on uncoded DNC error-rate performance at the relay. In addition to curves for three values of $N$, a curve is shown indicating the performance with perfect fading-amplitude knowledge. . . . .

2.5 Influence of fading-block length $N$ on turbo-coded DNC error-rate performance at the relay. Two curves are shown for each value of $N=\{8,16,32,64,128\}$. Solid curves denote perfect fading-amplitude knowledge. Dashed curves denote estimated fading amplitudes. . . . . . . . . . . . . . . . . .

2.6 SNR required to reach a bit error rate of $10^{-4}$ at the relay as a function of fading-block length. Three systems are shown: The noncoherent receiver with known and estimated $\left\{\alpha_{1}, \alpha_{2}\right\}$ and with no CSI. All systems use a turbo code

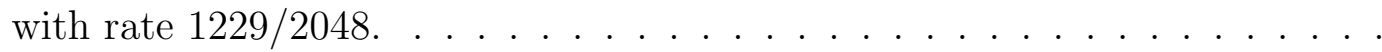

2.7 Comparison of error-rate performance between the turbo-coded DNC and LNC systems at the relay. The solid lines denote DNC, while the dashed lines denote LNC. . . . . . . . . . . . . . . . . . . . . . . . .

Comparison of the performance of turbo-coded DNC and LNC at the relay with block size $N=32$. For the DNC system, two code rates are shown, with the lower rate code offering comparable performance to the LNC system. . .

3.1 System Model for DNC two-way relay channel multiple-access phase with iterative decoding. . . . . . . . . . . . . . . . . . . . . . 
3.2 Frame structure for digital and link-layer network coding (DNC and LNC) during the TWRC multiple-access phase. For DNC, the end nodes each transmit $L$ symbols simultaneously. For LNC, each end node transmits $L / 2$ symbols in separate time slots. . . . . . . . . . . . . . . . . . .

3.3 Capacity for the TWRC MA phase in AWGN with random phase noise. Solid and dashed lines denote DNC and LNC respectively. Modulation orders are $M=\{2,4,8\}$.

3.4 Capacity for the TWRC MA phase in Rayleigh fading. Modulation orders are $M=\{2,4,8\}$. For DNC and LNC at every modulation order, a pair of similar curves is shown. Within each pair, the upper and lower curves depict capacity for partial and no CSI at the relay, respectively. . . . . . . . . . . .

3.5 End-to-end capacity in AWGN and Rayleigh fading with no CSI and partial CSI for digital and link-layer network coding (DNC and LNC). Modulation order $M=4$ is shown. . . . . . . . . . . . . . . . . . . .

3.6 LDPC-coded BER performance at the relay for digital network coding in AWGN and Rayleigh fading channels using a DVB-S2 LDPC code. The code length and rate are $N_{D}=16200$ bits and $r_{D}=3 / 5$ respectively. FSK modulation orders $M=\{2,4,8\}$ are simulated. In fading, performance with partial and no channel state information at the relay is shown. . . . . . . . . .

3.7 LDPC-coded BER performance at the relay for digital and link-layer network coding (DNC and LNC) in Rayleigh fading at channel code rates $r_{M}=$ $\{2 / 5,1 / 3\}$. The relay possesses partial CSI as fading amplitudes. The DNC and LNC frame lengths and rates are $N_{D}=16200$ and $N_{L}=8100$ bits. FSK

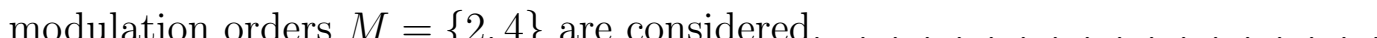

4.1 Example EXIT fit - DVB-S2 constraint . . . . . . . . . . . . . . .

4.2 DVB-S2-inspired LDPC-coded BER performance at the relay using optimized channel codes for DNC. The channel code rate is $r_{D}=r_{M}=3 / 5$. Performance is simulated in AWGN and Rayleigh fading with no CSI at the relay. The frame length is $N_{D}=16200$ bits. FSK modulation orders $M=\{2,4,8\}$ are considered. . . . . . . . . . . . . . . . . . .

4.3 WiMAX-inspired LDPC-coded BER performance at the relay using optimized channel codes for DNC. The channel code rate, codeword length, and modulation order are $r_{D}=r_{M}=2 / 3, N=2304$ and $M=4$ respectively. A code from the WiMAX standard is simulated for comparison, denoted as "standard", while optimized codes are denoted by their degree distribution. Solid lines denote partial CSI at the relay, while dashed lines denote no CSI. . . .

4.4 Error rate performance for EXIT-optimized LDPC codes at the TWRC relay during the multiple access phase. The modulation order is $M=8$. See the caption to Fig. 4.3 for remaining parameters. 
5.1 System Model - Analog Network Coded Two-way Relay Channel. The configuration of End Node 2 is identical to 1, and has been omitted from the

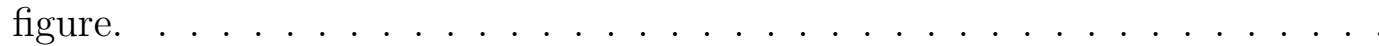

5.2 Bit error rate performance with no channel coding at the end node in the twoway relay channel broadcast stage under Rayleigh fading. The modulation orders considered are $M=\{2,4\}$. The number of demodulator infinite series terms considered are $N_{t}=\{5,15,25,50\}$. . . . . . . . . . . . . .

5.3 LDPC-coded bit error rate performance at as a function of demodulator infinite series terms. The LDPC code parameters are codeword length $L=16200$ and rate $r_{S}=1 / 2$. All simulations use BICM decoding. . . . . . . . . . .

5.4 LDPC-coded bit error rate performance as a function of decoder feedback (BICM vs BICM-ID). The LDPC code parameters are codeword length $L=$ 16200 and rate $r_{S}=1 / 2$. For all simulation $N_{t}=50 . \ldots$. . . . . . . . 88

6.1 Baseband Transmission Model . . . . . . . . . . . . . . . . . . . . . . 92

6.2 Simulated performance of noncoherent detection rules under oscillator offset. Blue, dashed lines denote the detection rule which does not model offset, while black, solid lines denote the detection rule which does model offset. Offset $d_{1}=0$ for all cases. . . . . . . . . . . . . . . . . . . . . . 100

6.3 Simulated performance of noncoherent detection rule incorporating frequency offset assuming nonzero offsets at both end nodes. Offset $d_{1}=0.04$ for all cases. 100

6.4 Simulated performance of noncoherent detection rule incorporating frequency offset assuming nonzero offsets at both end nodes. Offset $d_{1}=0$ for all cases. A rate $4500 / 6500$ turbo code is applied to all simulations. Blue, dashed lines denote the detection rule which does not model offset, while black, solid lines denote the detection rule which does model offset. . . . . . . . . . . . . . . . 102

6.5 System Model . . . . . . . . . . . . . . . . . . . . . . . . 105

6.6 Sphere Decoding Example: $\mathrm{M}=2$. . . . . . . . . . . . . . . . . . . . . . 111

6.7 Simulated error-rate performance for modulation order $M=2$. The number of sources considered is $K=\{2,3,4,5\}$. The information sequence length is $L=2304$. List sphere decoding uses $N_{S}=5$ symbols per list. A sphere decoding radius $r=4 N_{0}$ is utilized. . . . . . . . . . . . . . . 114

6.8 Simulated error-rate performance for modulation order $M=4$. See the Fig 6.7 caption or Section 6.2 .4 for simulation parameters. . . . . . . . . . . . . . 114 


\section{List of Tables}

4.1 Optimized LDPC variable node degrees based on DVB-S2 for code rate $r_{D}=$ $3 / 5$. The SNRs required to reach a BER of $10^{-4}$ for optimized and standard codes are given in columns opt Opt. and Std. respectively. For all codes $d_{v, 1}=2, o_{1}=6480$ and $d_{c}=11 \ldots \ldots \ldots \ldots$

4.2 Optimized LDPC variable node degrees based on DVB-S2 for code rate $r_{D}=$ $2 / 5$. For all codes $d_{v, 1}=2, o_{1}=9720$ and $d_{c}=6$. See caption on Table 4.1 for a full description. . . . . . . . . . . . . . . . . . .

4.3 Optimized LDPC variable node degrees based on WiMAX for code rate $r_{D}=$ $2 / 3$. The SNRs required to reach a BER of $10^{-4}$ for optimized and standard codes are given in columns opt Opt. and Std. respectively. For all codes $d_{v, 1}=2, o_{1}=672, d_{v, 2}=3, o_{2}=96$ and $d_{c}=10 \ldots \ldots \ldots$. . . . . . 71

6.1 Example values of Oscillator Offset . . . . . . . . . . . . . . 101 


\section{Notation}

We use the following notation and symbols throughout

$\begin{array}{ll}E[\cdot] & : \text { Expectation operator } \\ p(\cdot) & : \text { Probability density function }(\mathrm{pdf}) \\ P(\cdot) & : \text { Probability mass function }(\mathrm{pmf}) \\ \oplus & : \text { Binary exclusive-or operator } \\ \exp \{a\} & : e^{a} \\ \log (\cdot) & : \text { Natural logarithm } \\ \log _{2}(\cdot) & : \text { Logarithm with base } 2 \\ \operatorname{diag}(a, b, \ldots) & : \text { Diagonal matrix with entries } a, b, \ldots \\ {[\cdot]^{T}} & : \text { Matrix/vector transpose }\end{array}$

Bold upper case letters denote matrices and bold lower case letters denote vectors. 


\section{Chapter 1}

\section{Introduction}

This chapter provides an introduction to the contributions presented in this dissertation. Context is provided to motivate the underlying goals and assumptions. A conceptual description and review of relevant literature is provided for the primary technical concepts. The system modeling assumptions made throughout are described.

\subsection{Context}

The majority of modern wireless communication systems are designed to avoid interference between wireless devices (nodes) by assigning different resources to transmitting nodes. For example, fourth generation $(4 \mathrm{G})$ cellular networks divide the available frequency bands and transmission times between groups of phones within a cell, and transmissions from each group to the base station are separated at the base station using multi-antenna techniques [1]. In general, interference can be avoided by dividing transmissions in time, space, frequency or through signal processing techniques that separate multiple signals at a receiver. Examples of wireless communication systems designed to avoid interference are shown in Fig. 1.1.

Now suppose that a wireless system contains multiple transmitting and receiving nodes,

and assumptions such as interference avoidance are relaxed. How can the system be designed to maximize performance? A general description of the performance limits for multi-node wireless communications gives rise to network information theory [2]. Performance limits 


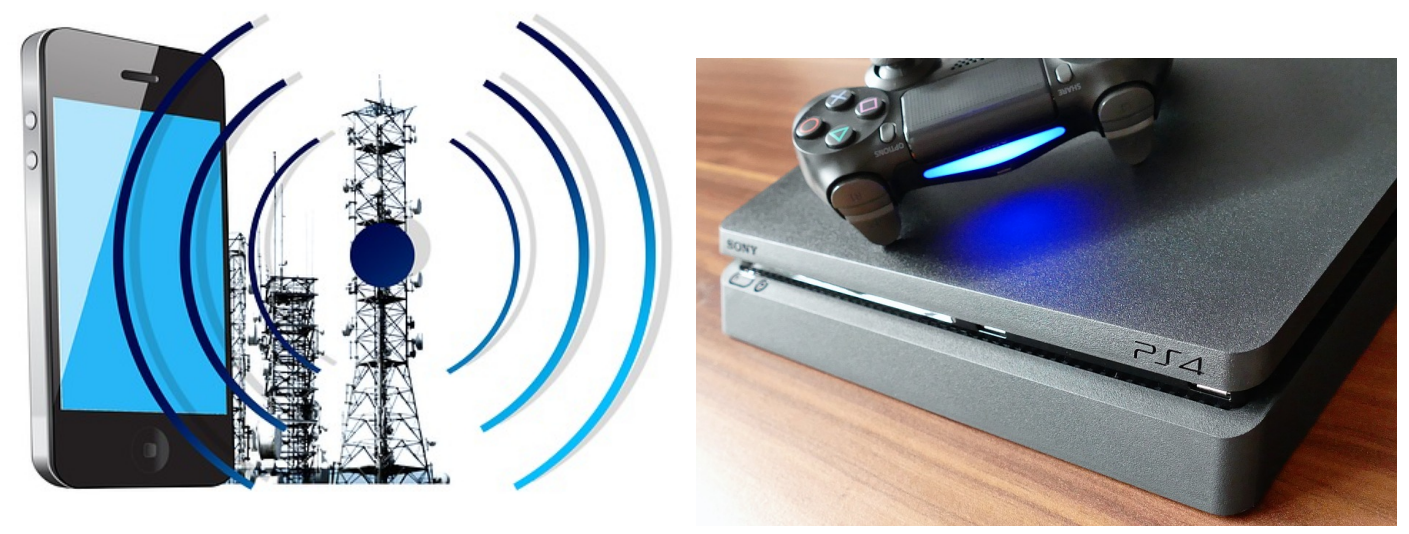

(a) Cellular phone communicating with tower. (b) Sony Playstation 4 console and controller.

Figure 1.1: Examples of wireless communication.

are known for some special cases, such as the multiple-access channel, where several nodes transmit simultaneously to one node. Considering the broadcast channel, where one node transmits separate information for many nodes using a common signal, only a partial description of the performance limits is known.

Consider a scenario where two wireless nodes wish to exchange information, but are outside radio range of each other, and another nodes lies in between them, that may act as a relay. An example of this scenario is two mobile users video chatting while connected to the same cellular tower. In terms of network information theory, this scenario is referred to as the two-way relay channel (TWRC) and is the subject of intense research effort [3]. The nodes exchanging information are referred to as the end nodes while the node performing relaying is referred to as the relay node. The two-way relay channel is depicted graphically in Fig. 1.2(a).

There are a variety of ways to implement communication in the TWRC. The most obvious is to model the information exchange as a series of point-to-point links, where the end nodes and relay transmit using entirely separate channel resources. For example, consider a system where the nodes transmit in separate time slots. The transmission schedule for a single TWRC exchange where the nodes use separate time slots is shown in Fig. 1.2(b). End nodes $\mathcal{N}_{1}$ and $\mathcal{N}_{2}$ exchange bits $b_{1}$ and $b_{2}$ respectively in four time slots. A transmission 


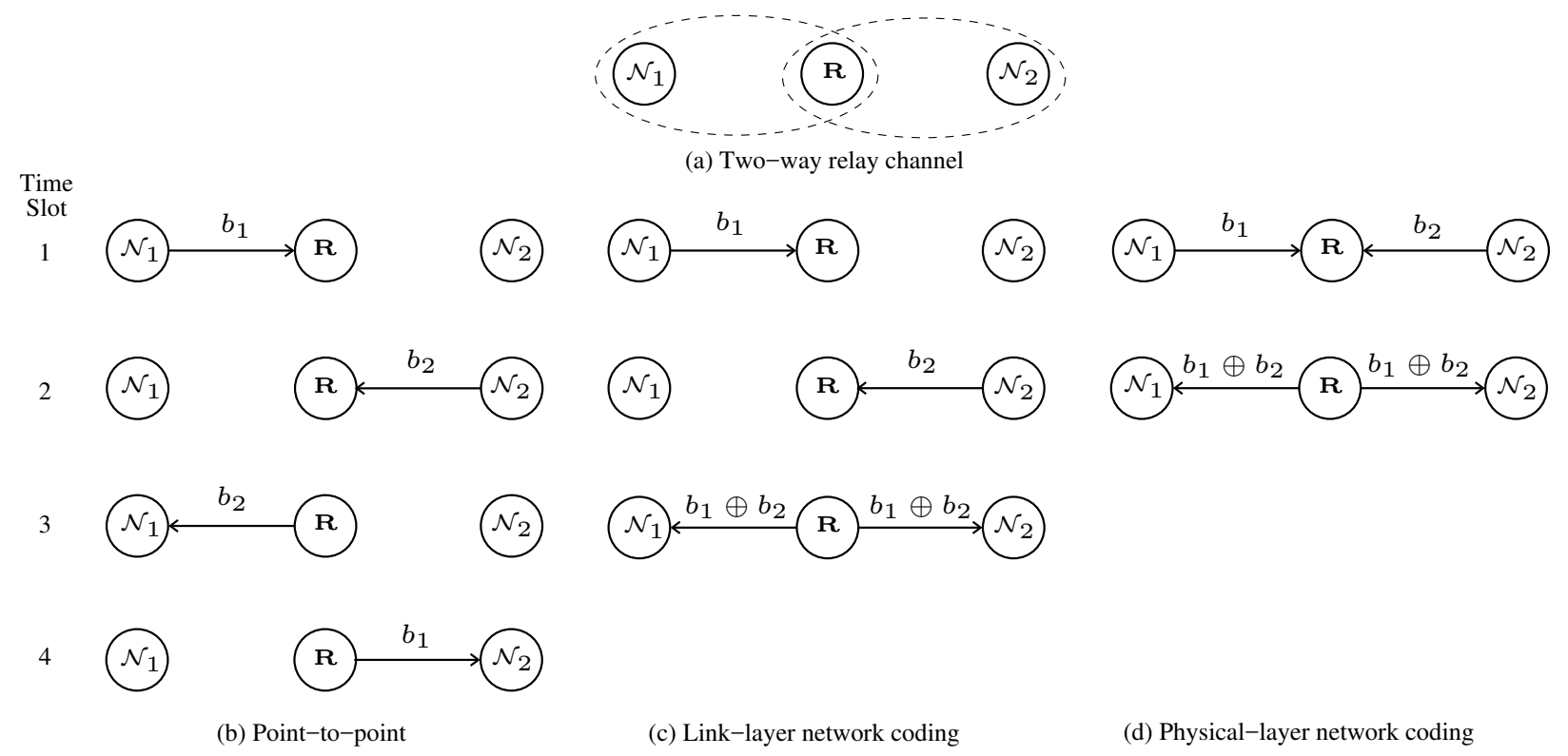

Figure 1.2: Two-way relay channel (a) and schedules for several exchange techniques: All links modeled as point-to-point (b), link-layer network coding (c), physical-layer network $\operatorname{coding}(\mathrm{d})$.

step can be saved by recognizing that the relay can combine information from the end nodes such that the end nodes can resolve the combined information, an operation referred to as link-layer network coding [4]. The transmission schedule for a single exchange in the networkcoded TWRC is shown in Fig. 1.2(b). After receiving bits $b_{1}$ and $b_{2}$ from the end nodes, the relay combines the bits by exclusive-or as $b_{1} \oplus b_{2}$ and broadcasts to the end nodes. After receiving the combined bits, each end node recovers the bit transmitted by the opposite end node by computing the exclusive-or of its own bit with the received bit (for example, node $\mathcal{N}_{1}$ computes $b_{2}=b_{1} \oplus\left(b_{1} \oplus b_{2}\right)$.

\subsubsection{Physical Layer Network Coding}

Physical-layer network coding (PNC) [5] [6] is a transmission scheme which reduces the number of time slots required for information exchange even further than link-layer network coding. The key feature of PNC is that the end nodes transmit to the relay at the same time in the same band, deliberately causing interference between their transmitted signals. 
The relay computes $b_{1} \oplus b_{2}$ directly from the interfered signals transmitted by the end nodes. This deliberate interference saves a time step versus link-layer network coding, reducing the number of required time slots from three to two, as shown in Fig. 1.2(d). The first time step involves two end nodes transmitting to the relay node, and thus is referred to as the multiple-access (MA) stage, also referred to as the uplink stage. In the second time step, the relay broadcasts a signal to both end nodes, and is referred to as the broadcast (BC) stage, at times referred to as the downlink. PNC strategies supporting more than three nodes have been developed [7] [8], however, in this dissertation, we focus on the case containing two source (end) nodes and one relay.

PNC may be broadly categorized based on the relay forwarding technique [9]. We consider two relaying schemes: analog network coding (ANC) [10] and digital network coding (DNC). In ANC, the relay forwards the received signal sum directly and all of the processing is performed at the end nodes. While the benefit of ANC is a simple relay implementation, the disadvantage is that the noise at the relay is also forwarded to the end nodes, potentially degrading performance and having high processing requirements. In DNC, the relay performs detection of the network-coded bits, essentially mitigating the effects of noise. It then remodulates the signal and broadcasts to the end nodes. The benefit of DNC is that the noise received at the relay is not retransmitted and the terminal receivers are simplified, but the disadvantage is that a more complex receiver is required at the relay. Thus, a crucial aspect of implementing PNC is the formulation of an efficient relay receiver, and the selection of coded-modulation formats that work well in DNC and ANC.

There are several challenges to implementing PNC in the two-way relay channel. In the ideal case, the symbols transmitted by the end nodes to the relay in the two-way relay channel MA stage would be received at the relay perfectly synchronized in time. The reception times for both symbols can be synchronized coarsely by network timing updates, however, there will almost certainly be slight timing offsets between symbols. A major point of research interest is examining and compensating for the effects of symbol timing offsets in the PNC multiple-access stage. In [11], a general algorithm for decoding in the MA stage in the 
presence of symbol asynchrony using belief propagation is developed, and it is demonstrated through simulation that the performance penalty can be almost completely eliminated. A generalization of the sum-product algorithm, that takes into account symbol asynchronism in the MA stage, is developed in [12] for decoding LDPC codes. An LDPC decoding algorithm for the MA stage is developed by [13] by taking the offset into account in the formulation of the bitwise LLRs. The work in [14] develops quasi-cyclic channel codes that can be decoded even in the presence of MA stage asynchronism. Implementations of PNC using software-defined radios are described in [15] and [16].

\subsubsection{Noncoherent Frequency Shift Keying}

Many modulation schemes require at the receiver exact knowledge of the transmitted signal phase, referred to as coherent demodulation. There are many circumstances where coherent demodulation is impractical due to difficulties acquiring the signal phase, for example, sensor networks that use inexpensive, imprecise oscillators which produce phase noise, military systems using fast frequency hopping [17], and fast-moving receivers such as missiles. These difficulties motivate the development of schemes that do not require exact carrier phase knowledge at the receiver, known as noncoherent demodulation.

In this dissertation, we develop a noncoherent form of PNC using $M$-ary frequency shift keying (M-FSK) modulation. FSK is attractive in scenarios where phase noise and unstable carrier frequencies occur, since it can be noncoherently detected. Ideally both end nodes transmit with the same carrier frequency. However, due to instabilities in the node's oscillators and different Doppler shifts due to independent motion, it is not feasible to assume that these two frequencies are the same at the relay receiver. At best, the relay receiver could lock onto one of the two frequencies, in which case the received phase of the other signal would drift from one symbol to the next.

Fundamentally, M-FSK modulation is implemented by varying the carrier frequency of the signal transmitted by the source between $M$ states, referred to as tones [18], according to the data to be transmitted. The receiver for M-FSK may be implemented as a bank 
of $M$ correlators, each having an oscillator tuned to the corresponding tone. We assume that the spacing between the tones is such that each correlator only detects energy for the tone to which it is matched, referred to as orthogonal tone spacing. Since we consider noncoherent demodulation, the minimum required frequency spacing between each tone is the inverse of the symbol period. An M-FSK transmitter can be implemented using $M$ separate oscillators by abruptly switching between the oscillators according to the tone to be transmitted. Abrupt switching yields a transmitted signal having discontinuities, yielding significant power in the spectral side-lobes. Side-lobe power can be reduced by varying the tones such that the transmitted signal is continuous, referred to as continuous-phase frequency shift keying (CPFSK). CPFSK exhibits more compact spectrum use than noncontinuous FSK [18].

A graphical depiction of a correlation-type detector for noncoherent binary FSK $(M=2)$ is shown in Fig. 1.3 [18]. The carrier frequency is $f_{c}$ and the frequency spacing between each tone is $f_{d}=1 / T$, where $T$ is the symbol period. The received signal $r(t)$ is correlated against the in-phase and quadrature components tuned to the frequencies for both tones to produce sample metrics $r_{1 c}, r_{1 s}, r_{2 c}$ and $r_{2 s}$. The sample metrics for each tone are squared and added, $r_{1}=r_{1 c}^{2}+r_{1 s}^{2}$ for tone 1 and $r_{2}=r_{2 c}^{2}+r_{2 s}^{2}$ for tone 2. A decision metric is computed as $r=r_{1}-r_{2}$, and the receiver decides that tone 1 was transmitted if $r$ is greater than zero and tone 2 if $r$ is less than zero.

It is commonly assumed in the PNC literature that signals are coherently demodulated and that perfect channel-state information (CSI) is available at the receivers. For instance, decode-and-forward relaying has been considered for binary phase-shift keying [19] and minimum-shift keying [20] modulations, but in both cases the relay must perform coherent reception. An amplify-and-forward protocol is considered in [21], which allows the decision to be deferred by the relay to the end-node, though detection is still coherent. When two signals arrive concurrently at a common receiver, neither coherent detection nor the cophasing of the two signals (so that they arrive with a constant phase offset) is practical. The latter would require preambles that detract from the overall throughput, stable 


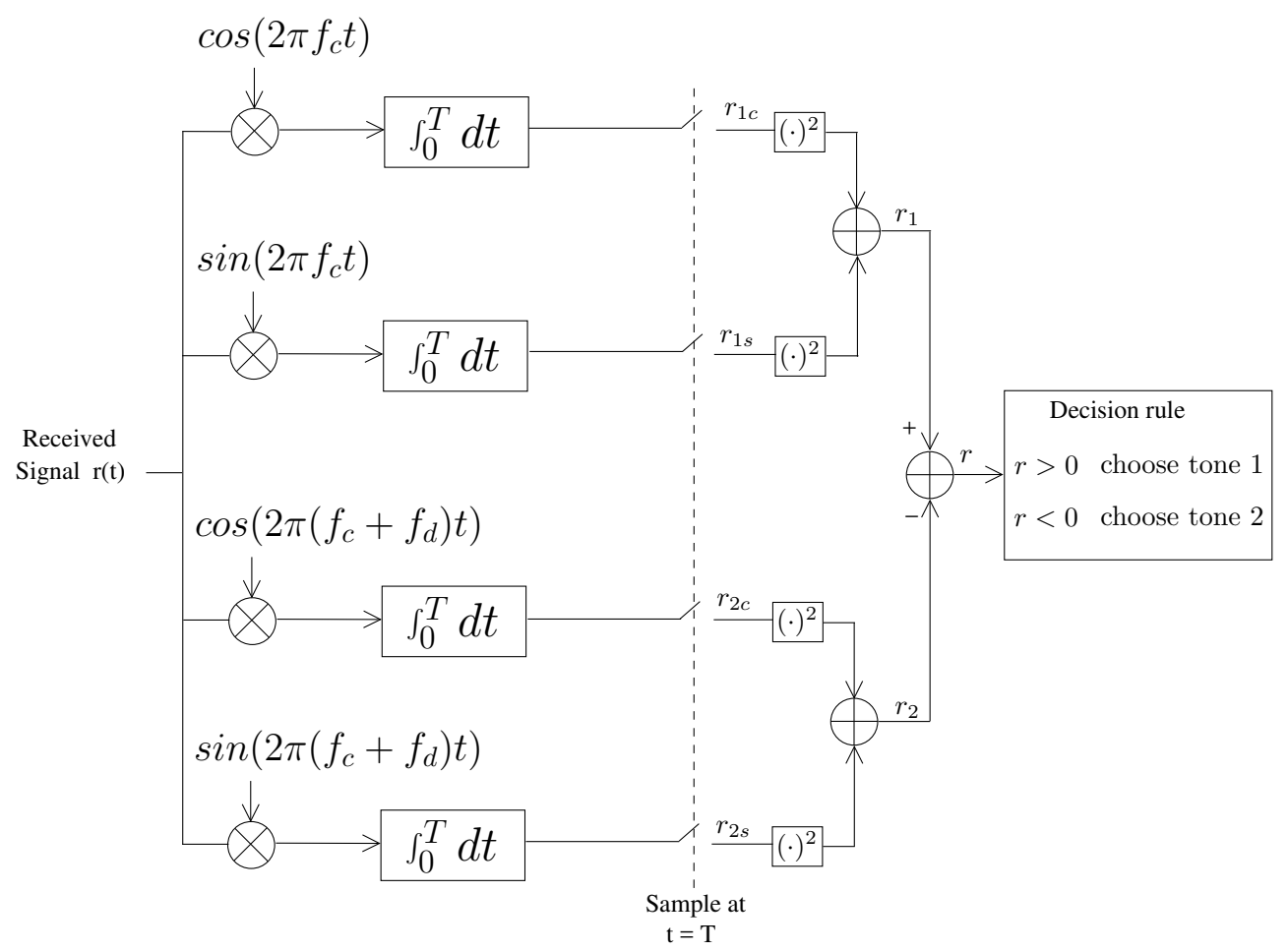

Figure 1.3: Correlation-type detector for noncoherent binary frequency-shift keying.

phases, and small frequency mismatches. To solve this problem, FSK for PNC was proposed for DNC systems in [22] and [23]. An alternative to noncoherent FSK is to use differential modulation, which has been explored in [24] and [25].

A noncoherent binary FSK detector for PNC is developed in [26] with capacity and bit error rate analysis presented for additive white Gaussian noise (AWGN) channels, and a noncoherent detector for binary continuous-phase binary FSK performing PNC in AWGN accounting for carrier phase offset is presented in [27]. As a further example of the application of FSK to the DNC uplink, the bit error rate and capacity for coherently-detected M-ary FSK at the DNC relay in AWGN is analyzed in [28]. However, this prior art has focused on either binary FSK or coherent M-ary FSK. To our knowledge, no prior work (other than our related conference papers [29-31]) has considered noncoherent M-FSK for the DNC uplink, which is our focus. Noncoherent reception is essential for the aforementioned reasons, while, as we show, usage of M-FSK provides important additional gains in energy efficiency over 
binary FSK. In the case of modulation order four $(M=4)$, the gain in energy efficiency comes without a requirement for additional bandwidth.

\subsubsection{Channel Coding}

When information transmitted by a source traverses a channel it can be corrupted by effects such as thermal noise, fading, and Doppler shifts. Channel coding is a technique to protect transmitted information against corruption by introducing redundancy into the transmission. The landmark contribution by Shannon [32] proved that information can be transmitted over a noisy channel with arbitrarily low probability of error by channel coding, provided that the rate of transmission is within the channel capacity. A major objective of this dissertation is to develop PNC systems capable of taking advantage of modern channel coding techniques, namely turbo and low-density parity check (LDPC) coding [33]. We develop demodulators that produce log-likelihood ratios (LLRs) for channel-coded bits suitable for use with these codes. In general, our coding and modulation framework is bit-interleaved coded modulation [34] with iterative decoding [35] (BICM-ID) where information is fed back from decoder to demodulator to refine symbol likelihoods and improve decoding performance.

Combining PNC with channel coding yields a throughput improvement while protecting against errors introduced by the channel. With regards to DNC, there are several approaches to applying channel coding [36]. Performing channel decoding at both the relay and at the end nodes is termed link-by-link channel coding (ANC systems must necessarily perform decoding only at the end nodes). When the channel codes are linear and the same codebook utilized by both users, the relay receiver decodes the modulo- 2 sum of the two transmitted codewords (i.e., the network-coded codeword) which is itself a codeword in the same codebook. The received network-coded codeword can then be passed through a standard binary channel decoder to extract the network-coded message, which due to the linearity of the channel code will be the modulo-2 sum of the two users' messages. The network-coded message can again be channel-coded using the same or a different code and then broadcast to the two users. While the modulo-2 summation has been shown to discard information during 
demodulation, applying iterative decoding between the decoder and demodulator can mitigate some information loss [37]. Additionally, performing decoding over the network-coded bits allows the use of powerful and flexible binary channel coding techniques.

It has been recognized that optimizing channel codes for specific channels yields performance benefits [38]. There are a variety of approaches to code optimization for the physicallayer-network-coded TWRC. In [39], LDPC codes are optimized by identifying parity check matrix column weights and removing graph cycles. LDPC codes are optimized by identifying degree distributions that minimize probability of decoding error in [40], [41] and [42]. In [43], novel protographs are developed to construct LDPC codes exhibiting capacity-approaching performance In this dissertation we optimize LDPC codes for the DNC multiple-access stage using extrinsic information transfer charts (EXIT) [44] to identify degree distributions that improve performance over standard codes.

\subsection{Summary of Contributions}

This section summarizes the contributions described in this dissertation. All of the contributions listed have either been peer-reviewed or are under review at the time of this writing. The chapters where each is developed are listed. The specific contributions are

1. We formulate a soft-output noncoherent DNC relay demodulator for M-ary FSK supporting iterative [30] and noniterative decoding [23] [29] (chapters 2 and 3). The demodulator is formulated assuming several cases of available channel state information.

2. We consider the use of a turbo code for data protection [23] [45]. This requires that the relay receiver be formulated so that it produces bitwise LLRs, which may be decoded using a standard turbo decoder (chapter 2).

3. We formulate a channel estimator for the DNC multiple-access stage that estimates the amplitudes of the fading coefficients encountered by the symbols transmitted from 
the end nodes to the relay [45] [46]. Error-rate performance using estimation is compared against the cases where the receiver has perfect and no amplitude knowledge. Performance is also measured as a function of Rayleigh fading block length (chapter 2).

4. We perform a capacity analysis of the noncoherent DNC multiple-access [29] [30] and broadcast stages [47], providing a theoretical description of end-to-end performance. The capacity analysis accurately predicts the performance of the system when using optimized codes. LNC uplink and downlink is analyzed in order to identify scenarios where DNC and LNC exhibit the best performance (chapter 3).

5. We optimize the LDPC codes used on the DNC uplink by identifying appropriate variable node degree distributions using EXIT charts [31]. The optimized codes demonstrate a significant improvement over well-known commercialized LDPC codes designed for point-to-point channels (chapter 4).

6. We formulate an M-ary FSK receiver for the end nodes in the ANC two-way relay channel [48]. The receiver supports feedback from decoder to demodulator to refine the symbol likelihoods (BICM-ID). Formulation of the demodulator leads to an infinite summation, which is truncated for implementation. Bit-error rate performance of the demodulator is investigated with and without LDPC channel coding (chapter 5).

7. Other contributions include analysis and simulation of the performance of binary FSK in the DNC multiple-access stage under frequency offset [49], and reduced complexity decoding the DNC multiple-access stage using sphere decoding [50] (chapter 6).

\subsection{System Model Elements used Throughout}

This section describes the system modeling assumptions that apply throughout the dissertation. Details that are specific to the contribution in each chapter are described in the chapter's system model section. Node transmission and channel details are described, as 
well as general details of node reception. The transmission schemes for DNC and LNC are described. The subscript $P$ denotes a parameter having value that depends on the network coding scheme, for example, $r_{P}$ denotes a channel code rate taking value $r_{D}$ for DNC and $r_{L}$ for LNC.

\subsubsection{Transmission by End Nodes}

The end nodes $\mathcal{N}_{i}, i \in\{1,2\}$ generate binary information sequences $\mathbf{u}_{i}=\left[u_{1, i}, \ldots, u_{K, i}\right]$ having length $K$. Each $\mathbf{u}_{i}$ is encoded by a rate- $r_{P}$ linear block code yielding a length $N_{P}=$ $K / r_{P}$ channel codeword, denoted by $\mathbf{b}_{i}^{\prime}=\left[b_{1, i} \ldots, b_{N_{P}, i}\right]$. The codeword is passed through an interleaver, modeled as a permutation matrix $\Pi$ having dimensionality $N_{P} \times N_{P}: \mathbf{b}_{i}=\mathbf{b}_{i}^{\prime} \Pi$. Let $\mathcal{D}=\{0, \ldots, M-1\}$ denote the set of integer indices corresponding to each FSK tone, where $M$ is the modulation order. The number of bits per symbol is $\mu=\log _{2} M$. The codeword $\mathbf{b}_{i}$ at each node is divided into $L_{P}=N_{P} / \mu$ sets of bits, each of which is mapped to an $M$-ary symbol $q_{k, i} \in \mathcal{D}$, where $k$ denotes the symbol index, and $i$ denotes the node.

The modulated signal transmitted by end node $\mathcal{N}_{i}$ during signaling interval $k T_{s} \leq t<$ $(k+1) T_{s}$ is

$$
s_{k, i}(t)=\sqrt{\frac{2}{T_{s}}} \cos \left[2 \pi\left(f+\frac{q_{k, i}}{T_{s}}\right)\left(t-k T_{s}\right)\right]
$$

where $f$ is the end node carrier frequency and $T_{s}$ is the symbol period. We assume a vector channel model where the vector dimensions correspond to matched filter outputs, each representing a particular frequency. The transmitted symbol vectors are represented by the set of column vectors $\mathbf{x}_{k, i}$. Each $\mathbf{x}_{k, i}$ is length $M$, contains a 1 at vector position $q_{k, i}$, and 0 elsewhere. The modulated codeword from end node $\mathcal{N}_{i}$ is represented by the matrix of symbols $\mathbf{X}_{i}=\left[\mathbf{x}_{1, i}, \ldots, \mathbf{x}_{L_{P}, i}\right]$, having dimensionality $M \times L_{P}$.

\subsubsection{Channel Model for Multiple-Access Stage}

We consider two noncoherent channel models. The first is a frequency-flat Rayleigh fading channel having independent gains for every symbol period. The second is an additive white 
Gaussian noise channel (AWGN) that corrupts the symbol phase. The gain from node $\mathcal{N}_{i}$ to the relay during a particular signaling interval $k$ is denoted by $h_{k, i, R}$. The gain is represented as $h_{k, i, R}=\alpha_{k, i, R} e^{j \theta_{k, i, R}}$, where $\alpha_{k, i, R}$ is Rayleigh distributed for the fading channel and unity for AWGN. To model the lack of phase synchronization between the end nodes and relay as described in Section 1.1.2, we let the phase shift within a block vary independently from symbol to symbol. Term $\theta_{k, i, R}$ is the phase, uniformly distributed between $[0,2 \pi)$. In the fading model, the amplitudes are selected such that the received energy at the relay from node $\mathcal{N}_{i}$ is $\mathcal{E}_{i}$

$$
E\left[\left|h_{k, i, R}\right|^{2}\right]=E\left[\alpha_{k, i, R}^{2}\right]=\mathcal{E}_{i} .
$$

Consider symbol transmission from the end nodes to the relay during the MA stage. In DNC, the end nodes transmit simultaneously to the relay over the same time and band. It is assumed that the frames transmitted by the end nodes are received perfectly synchronized at the relay receiver. The frame received at the relay assuming DNC is

$$
\mathbf{Y}_{R}=\mathbf{X}_{1} \mathbf{H}_{1, R}+\mathbf{X}_{2} \mathbf{H}_{2, R}+\mathbf{N}_{R}
$$

Considering LNC, the end nodes transmit in separate time slots to the relay. The received frames are

$$
\begin{gathered}
\mathbf{Y}_{1, R}=\mathbf{X}_{1} \mathbf{H}_{1, R}+\mathbf{N}_{1, R} \\
\mathbf{Y}_{2, R}=\mathbf{X}_{2} \mathbf{H}_{2, R}+\mathbf{N}_{2, R}
\end{gathered}
$$

where $\mathbf{H}_{i, R}$ is an $L_{P} \times L_{P}$ diagonal matrix of channel coefficients having value $h_{k, i, R}$ at matrix entry $(n, n)$ and 0 elsewhere, and $\mathbf{N}_{R}$ and $\mathbf{N}_{i, R}$ are $M \times L_{P}$ noise matrices. Denote a single column of $\mathbf{N}_{R}$ and $\mathbf{N}_{i, R}$ as $\mathbf{n}_{k, R}$ and $\mathbf{n}_{k, i, R}$ respectively. Each column is composed of zeromean circularly symmetric complex jointly Gaussian random variables having covariance matrix $N_{0} \mathbf{I}_{M}$; i.e., $\mathbf{n}_{k} \sim \mathcal{N}_{c}\left(\mathbf{0}, N_{0} \mathbf{I}_{M}\right) . N_{0}$ is the one-sided noise spectral density, and $\mathbf{I}_{M}$ is the $M$-by- $M$ identity matrix. Single columns of $\mathbf{Y}_{R}$ and $\mathbf{Y}_{i, R}$ represent a channel observation and are denoted by

$$
\mathbf{y}_{k, R}=\mathbf{h}_{k, 1, R} \mathbf{x}_{k, 1}+\mathbf{h}_{k, 2, R} \mathbf{x}_{k, 2}+\mathbf{n}_{k, R}
$$


in the PNC model and

$$
\begin{array}{r}
\mathbf{y}_{k, 1, R}=\mathbf{h}_{k, 1, R} \mathbf{x}_{k, 1}+\mathbf{n}_{k, 1, R} \\
\mathbf{y}_{k, 2, R}=\mathbf{h}_{k, 2, R} \mathbf{x}_{k, 2}+\mathbf{n}_{k, 2, R}
\end{array}
$$

in LNC.

\subsection{Conclusion}

This chapter has provided an introduction for the contributions made in this dissertation. The fundamental aspects of physical-layer network coding were described and placed within the broader context of network information theory. Motivations for considering frequencyshift keying modulation were provided. A key element of the developed physical-layer network-coded systems developed is their ability to perform signal detection in the presence of carrier phase instability and lack of phase synchronization, referred to as noncoherent detection. The contributions presented in this dissertation were outlined, with references to publication in peer-reviewed venues. The system model elements used throughout the dissertation were described. 


\section{Chapter 2}

\section{Noncoherent Binary FSK System for DNC}

This chapter develops a noncoherent soft-output binary frequency-shift keying (FSK) demodulator for the relay in the digital-network-coded (DNC) two-way relay channel (TWRC). We focus on non-iterative binary FSK in this chapter as a fundamental step towards developing more sophisticated demodulator formulations. The demodulator produces log-likelihood ratios (LLRs) suitable for use with iterative channel coding techniques. The demodulator is formulated for several cases of channel state information (CSI). We develop a channel estimator that estimates the values of the fading amplitudes between the end nodes and relay. The performance of the demodulator is simulated with and without turbo channel decoding. DNC performance is compared to link-layer network coding (LNC), providing insight into cases where each is desirable.

\subsection{Introduction}

Performing channel coding in the PNC multiple-access (MA) stage protects transmitted data against channel errors and improves energy efficiency. The combination of channel coding and physical-layer network coding is considered in [36] and [51]. In [52], a bitinterleaved coded modulation (BICM) based soft-output demodulator is developed assuming 
phase-shift keying modulation, and performance is examined when coupled with a turbo channel code. In our first conference publication [23] we investigated the use of a turbo code in a noncoherent PNC system using FSK. When using a turbo code, the relay demodulator must be able to produce bitwise log-likelihood ratios (LLRs) that are passed as input to the channel decoder. The work in [53] proves that the error rate for the PNC multiple-access stage using exclusive-or based PNC mapping can achieve the same error rate as a maximumlikelihood detector that decodes by considering all possible codeword pairs transmitted by the end nodes.

Channel estimation is an important issue, especially when a channel code is used. A training-based channel estimation scheme for PNC at the relay assuming amplify-and-forward operation is considered in [54]. The relay estimates channel parameters from training symbols and adapts its broadcast power in order to maximize the signal-to-noise ratio at the end nodes. Estimation of both channel gains in the two-way relay channel at the end nodes, rather than the relay, is considered in [55]. Novel channel estimators are presented which provide better performance than common techniques such as least-square and linear-minimummean-squared error estimation. A channel estimation technique is developed for systems using orthogonal modulation is developed in [56], where the need for pilot symbols is eliminated by varying one user's symbol constellation during each symbol period. The work in [56] is extended in [57] by separating the symbol periods into cases where the end nodes transmit the same symbols and different symbols, and using the different symbol cases to estimate the fading gains over each node's channel. In [45], we propose a blind channel estimator for the relay of the noncoherent PNC system.

In this chapter, we investigate receiver-design issues encountered when analyzing noncoherent FSK for DNC systems. While noncoherent FSK has been previously proposed for DNC systems in [22], we make the following specific contributions:

1. We provide closed-form expressions for the relay receiver decision rule with different types of CSI. This is in contrast with [22], which resorted to numerical methods to solve the decision rule (see the comment below equation (8) in [22]). 
2. We consider the use of a turbo code for additional data protection. This requires that the relay receiver be formulated so that it produces bitwise LLRS, which may be passed through a standard turbo decoder.

3. We provide results for Rayleigh block-fading channels. The results in [22] were only for a phase-fading channel.

4. We propose a channel estimator which is capable of determining the fading amplitudes of the channels from the two terminals to the relay. The estimator does not require pilot symbols.

The remainder of this chapter is organized as follows. Section 2.2 presents the system modeling assumptions specific to this chapter. Section 2.3 presents the demodulator derivation, while Section 2.4 discusses channel-estimation issues. Section 2.5 provides simulation results, and Section 2.6 concludes the chapter.

\subsection{System Model}

The general channel model described in Section 1.3 considers fully-interleaved Rayleigh fading, where each transmitted symbol experiences an independent channel gain. In this chapter we extended the general model by considering block-fading. The system model is depicted graphically in Fig. 2.1. A block is defined as a set of $N$ symbols that all experience the same fading gain. The duration of each block corresponds roughly to the channel coherence time. The signal matrix $\mathbf{X}_{i}$ modeling the signals transmitted by node $\mathcal{N}_{i}$ may be partitioned into $N_{b}=L_{S} / N$ blocks according to

$$
\mathbf{X}_{i}=\left[\begin{array}{lll}
\mathbf{X}_{i}^{(1)} & \ldots & \mathbf{X}_{i}^{\left(N_{b}\right)}
\end{array}\right]
$$

where each block $\mathbf{X}_{i}^{(\ell)}, 1 \leq \ell \leq N_{b}$, is a $2 \times N$ matrix, and $N_{b}$ is assumed to be an integer. The channel associated with block $\mathbf{X}_{i}^{(\ell)}$ is represented by the $N \times N$ diagonal matrix

$$
\mathbf{H}_{i}^{(\ell)}=\alpha_{i}^{(\ell)} \times \operatorname{diag}\left(\exp \left\{j \theta_{i, 1}^{(\ell)}\right\}, \ldots, \exp \left\{j \theta_{i, N}^{(\ell)}\right\}\right)
$$




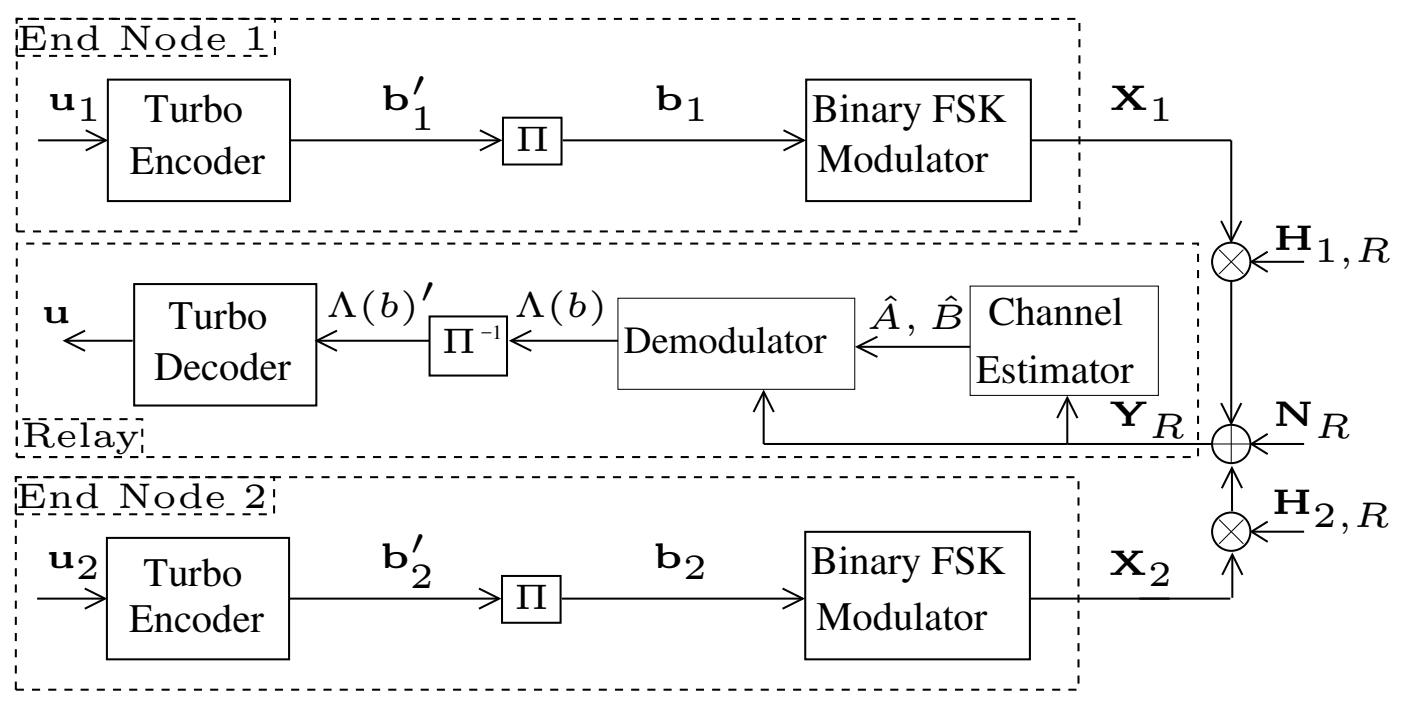

Figure 2.1: System Model for DNC two-way relay channel multiple-access stage.

where $\alpha_{i}^{(\ell)}$ is a real-valued fading amplitude and $\theta_{i, k}^{(\ell)}$ is the phase shift of the $k^{\text {th }}$ symbol. The $\theta_{i, k}^{(\ell)}$, s are i.i.d. uniform over the interval $[0,2 \pi)$. The energy transmitted by the end nodes is modeled as the variance of the fading amplitudes as described by Eq. (1.2). The $\ell^{\text {th }}$ block at the sampled output of the relay receiver's matched-filters is then

$$
\mathbf{Y}_{R}^{(\ell)}=\mathbf{X}_{1}^{(\ell)} \mathbf{H}_{1, R}^{(\ell)}+\mathbf{X}_{2}^{(\ell)} \mathbf{H}_{2, R}^{(\ell)}+\mathbf{N}_{R}^{(\ell)}
$$

where $\mathbf{N}_{R}^{(\ell)}$ is a $2 \times N$ noise matrix whose elements are i.i.d. circularly-symmetric complex Gaussian random variables with zero mean and variance $N_{0}$.

\subsection{Relay Receiver}

The goal of the relay receiver is to detect the network-coded combination of information bits transmitted by the end nodes, $\mathbf{u}=\mathbf{u}_{1} \oplus \mathbf{u}_{2}$. At the relay, each block $\mathbf{Y}_{R}^{(\ell)}$ of the channel observation matrix $\mathbf{Y}_{R}$ is passed to a channel estimator, which computes estimates of the fading amplitudes $\alpha_{1}^{(\ell)}$ and $\alpha_{2}^{(\ell)}$ as $A$ and $B$, as shown in Fig. 2.1. A full description of the estimator is given in Section 2.4.

The fading-amplitude estimates and channel observations are used to obtain soft estimates of the network-and-channel-coded bit sequence. The demodulator operates on a 
symbol-by-symbol basis, and therefore we may focus on a single signaling interval by dropping the dependence on the symbol interval $k$ and the block index $\ell$. Let $b_{1}$ and $b_{2}$ be the channel-coded bits transmitted by terminals $\mathcal{N}_{1}$ and $\mathcal{N}_{2}$ during a single signaling interval, and let $b=b_{1} \oplus b_{2}$ be the corresponding network-coded bit. The relay demodulator computes the LLR

$$
\Lambda(b)=\log \frac{P\left(b=1 \mid \mathbf{y}_{R}\right)}{P\left(b=0 \mid \mathbf{y}_{R}\right)}=\log \frac{P\left(b_{1} \oplus b_{2}=1 \mid \mathbf{y}_{R}\right)}{P\left(b_{1} \oplus b_{2}=0 \mid \mathbf{y}_{R}\right)}
$$

where $\mathbf{y}_{R}$ is the corresponding column of $\mathbf{Y}_{R}$. The event $\left\{b_{1} \oplus b_{2}=1\right\}$ is equivalent to the union of the events $\left\{b_{1}=0, b_{2}=1\right\}$ and $\left\{b_{1}=1, b_{2}=0\right\}$. Similarly, the event $\left\{b_{1} \oplus b_{2}=0\right\}$ is equivalent to the union of the events $\left\{b_{1}=0, b_{2}=0\right\}$ and $\left\{b_{1}=1, b_{2}=1\right\}$. It follows that

$$
\begin{aligned}
\Lambda(b) & =\log \frac{P\left(\left\{b_{1}=0, b_{2}=1\right\} \cup\left\{b_{1}=1, b_{2}=0\right\} \mid \mathbf{y}_{R}\right)}{P\left(\left\{b_{1}=0, b_{2}=0\right\} \cup\left\{b_{1}=1, b_{2}=1\right\} \mid \mathbf{y}_{R}\right)} \\
& =\log \frac{P\left(\left\{b_{1}=0, b_{2}=1\right\} \mid \mathbf{y}_{R}\right)+P\left(\left\{b_{1}=1, b_{2}=0\right\} \mid \mathbf{y}_{R}\right)}{P\left(\left\{b_{1}=0, b_{2}=0\right\} \mid \mathbf{y}_{R}\right)+P\left(\left\{b_{1}=1, b_{2}=1\right\} \mid \mathbf{y}_{R}\right)}
\end{aligned}
$$

where summations arise because the unions are taken over mutually exclusive events.

The LLRs produced by the demodulator are deinterleaved according to $\Lambda(b)^{\prime}=\Pi^{-1} \Lambda(b)$ and passed to the turbo channel decoder. The turbo decoder performs a specified number of iterations and then makes a hard decision on the network-coded data sequence, $\mathbf{u}$.

\subsubsection{Link-Layer Network Coding Receiver}

The LNC receiver operates on a symbol-by-symbol basis, so we may drop dependence on the symbol interval $k$ and block index $\ell$. In the LNC system, the LLR's of $b_{1}$ and $b_{2}$ are first computed independently during the orthogonal time slots and are then combined according to the rules of LLR arithmetic. The LLR of the signal sent from node $\mathcal{N}_{i}$ to the relay is

$$
\Lambda\left(b_{i}\right)=\log \frac{P\left(b_{i}=1 \mid \mathbf{y}_{i, R}\right)}{P\left(b_{i}=0 \mid \mathbf{y}_{i, R}\right)}
$$

where $\mathbf{y}_{i, R}$ is the signal received during the time slot that node $\mathcal{N}_{i}$ transmits. When the fading amplitudes $\alpha_{i}, i=1,2$, are known, but the phases $\theta_{i}, i=1,2$, are not known, then 
(2.6) is found using [58]

$$
\Lambda\left(b_{i}\right)=\log I_{0}\left(\frac{2 \sqrt{\mathcal{E}_{i}} \alpha_{i}\left|y_{2}\right|}{N_{0}}\right)-\log I_{0}\left(\frac{2 \sqrt{\mathcal{E}_{i}} \alpha_{i}\left|y_{1}\right|}{N_{0}}\right)
$$

where $I_{0}(\cdot)$ is the zeroth-order Bessel function of the first kind [59] and $y_{1}$ and $y_{2}$ are the components of $\mathbf{y}_{i, R}$. When the fading amplitudes are not known, but have Rayleigh distributions, then (2.6) is found using [58]

$$
\Lambda\left(b_{i}\right)=\frac{\left(\mathcal{E}_{i} / N_{0}\right)^{2}}{1+\mathcal{E}_{i} / N_{0}}\left\{\left|y_{2}\right|^{2}-\left|y_{1}\right|^{2}\right\} .
$$

Once the individual LLR's from each end node are found using (2.7) or (2.8), the LLR of the LNC system's network codeword can then be found from (2.5) and the independence of $b_{1}$ and $b_{2}$ when $\mathbf{y}_{i, R}$ is given:

$$
\begin{aligned}
\Lambda(b) & =\log \frac{e^{\Lambda\left(b_{1}\right)}+e^{\Lambda\left(b_{2}\right)}}{1+e^{\Lambda\left(b_{1}\right)+\Lambda\left(b_{2}\right)}} \\
& =\max *\left[\Lambda\left(b_{1}\right), \Lambda\left(b_{2}\right)\right]-\max *\left[0, \Lambda\left(b_{1}\right)+\Lambda\left(b_{2}\right)\right]
\end{aligned}
$$

where $\max *[x, y]=\log \left(e^{x}+e^{y}\right)$.

\subsubsection{Physical-Layer Network Coding Receiver}

The purpose of the PNC receiver is to directly compute the LLR of the network-coded bit $\Lambda(b)$, rather than $\Lambda\left(b_{1}\right)$ and $\Lambda\left(b_{2}\right)$ separately. We use (2.5) and assume that the four events are equally likely along with Bayes' rule to obtain

$$
\begin{aligned}
\Lambda(b)= & \log \left[p\left(\mathbf{y}_{R} \mid\left\{b_{1}=0, b_{2}=1\right\}\right)+p\left(\mathbf{y}_{R} \mid\left\{b_{1}=1, b_{2}=0\right\}\right)\right] \\
& -\log \left[p\left(\mathbf{y}_{R} \mid\left\{b_{1}=0, b_{2}=0\right\}\right)+p\left(\mathbf{y}_{R} \mid\left\{b_{1}=1, b_{2}=1\right\}\right)\right] .
\end{aligned}
$$

The computation of each $p\left(\mathbf{y}_{R} \mid\left\{b_{1}, b_{2}\right\}\right)$ for the PNC relay receiver given various levels of channel state information is the subject of the remainder of this section.

\section{Coherent PNC Receiver}

When the fading amplitudes and phases are known, $p\left(\mathbf{y}_{R} \mid\left\{b_{1}, b_{2}\right\}\right)$ is conditionally Gaussian. The mean is a two-dimensional complex vector whose value depends on the bits transmitted by the end nodes $\left\{b_{1}, b_{2}\right\}$ and the complex fading coefficients $\left\{h_{1}, h_{2}\right\}$, which are the 
corresponding entries of the fading matrix. Let $\mathbf{m}\left[b_{1}, b_{2}\right]$ be the mean of $\mathbf{y}_{R}$ for the given values of $b_{1}$ and $b_{2}$. When $b_{1} \neq b_{2}$, the two terminals transmit different frequencies and

$$
\begin{aligned}
\mathbf{m}[0,1] & =\left[\begin{array}{ll}
h_{1} & h_{2}
\end{array}\right]^{T} \\
\mathbf{m}[1,0] & =\left[\begin{array}{ll}
h_{2} & h_{1}
\end{array}\right]^{T} .
\end{aligned}
$$

When $b_{1}=b_{2}$, the two terminals transmit the same frequency and

$$
\begin{aligned}
\mathbf{m}[0,0] & =\left[\begin{array}{ll}
\left(h_{1}+h_{2}\right) & 0
\end{array}\right]^{T} \\
\mathbf{m}[1,1] & =\left[\begin{array}{ll}
0 & \left(h_{1}+h_{2}\right)
\end{array}\right]^{T} .
\end{aligned}
$$

Since there is a one-to-one correspondence between the event $\left\{b_{1}, b_{2}\right\}$ and the mean vector $\mathbf{m}\left[b_{1}, b_{2}\right]$, it is equivalent to write $p\left(\mathbf{y}_{R} \mid\left\{b_{1}, b_{2}\right\}\right)$ as $p\left(\mathbf{y}_{R} \mid \mathbf{m}\left[b_{1}, b_{2}\right]\right)$, where

$$
p\left(\mathbf{y}_{R} \mid \mathbf{m}\left[b_{1}, b_{2}\right]\right)=\left(\frac{1}{\pi N_{0}}\right)^{2} \exp \left\{-\frac{1}{N_{0}}\left\|\mathbf{y}-\mathbf{m}\left[b_{1}, b_{2}\right]\right\|^{2}\right\} .
$$

The coherent receiver computes each of the $p\left(\mathbf{y}_{R} \mid\left\{b_{1}, b_{2}\right\}\right)$ required by (2.10) by substituting the corresponding $\mathbf{m}\left[b_{1}, b_{2}\right]$ defined by (2.11) and (2.12) into (2.13).

\section{Noncoherent PNC Receiver with CSI}

Suppose that the receiver does not know the phases of the elements of the complexvalued $\mathbf{m}\left[b_{1}, b_{2}\right]$ vectors, but does know the magnitudes of the elements. The knowledge of the magnitudes constitutes a type of CSI. Define $\boldsymbol{\mu}\left[b_{1}, b_{2}\right]$ to be the two-dimensional real vector whose elements are the magnitudes of the elements of the complex vector $\mathbf{m}\left[b_{1}, b_{2}\right]$. When $b_{1} \neq b_{2}$, both frequencies are used, and

$$
\begin{aligned}
& \boldsymbol{\mu}[0,1]=\left[\begin{array}{ll}
\left|h_{1}\right| & \left|h_{2}\right|
\end{array}\right]^{T}=\left[\begin{array}{ll}
\alpha_{1} & \alpha_{2}
\end{array}\right]^{T} \\
& \boldsymbol{\mu}[1,0]=\left[\begin{array}{ll}
\left|h_{2}\right| & \left|h_{1}\right|
\end{array}\right]^{T}=\left[\begin{array}{ll}
\alpha_{2} & \alpha_{1}
\end{array}\right]^{T} .
\end{aligned}
$$

When $b_{1}=b_{2}$, only one frequency is used, and

$$
\begin{aligned}
& \boldsymbol{\mu}[0,0]=\left[\begin{array}{ll}
\left|h_{1}+h_{2}\right| & 0
\end{array}\right]^{T}=\left[\begin{array}{ll}
\alpha & 0
\end{array}\right]^{T} \\
& \boldsymbol{\mu}[1,1]=\left[\begin{array}{ll}
0 & \left|h_{1}+h_{2}\right|
\end{array}\right]^{T}=\left[\begin{array}{ll}
0 & \alpha
\end{array}\right]^{T}
\end{aligned}
$$


where we have defined $\alpha=\left|h_{1}+h_{2}\right|=\sqrt{\alpha_{1}^{2}+\alpha_{2}^{2}+2 \alpha_{1} \alpha_{2} \cos \left(\theta_{2}-\theta_{1}\right)}$.

The pdf of $\mathbf{y}_{R}$ conditioned on $\boldsymbol{\mu}\left[b_{1}, b_{2}\right]$ may be found by marginalizing over the unknown phases

$$
p\left(\mathbf{y}_{R} \mid \boldsymbol{\mu}\left[b_{1}, b_{2}\right]\right)=\int_{0}^{2 \pi} \int_{0}^{2 \pi} p\left(\phi_{1}, \phi_{2}\right) p\left(\mathbf{y}_{R} \mid \mathbf{m}\left[b_{1}, b_{2}\right]\right) d \phi_{1} d \phi_{2} .
$$

where $\phi_{1}$ and $\phi_{2}$ are the phases of the first and second elements of $\mathbf{m}\left[b_{1}, b_{2}\right]$, respectively.

Assume that the $\alpha_{i}$ are Rayleigh distributed so that the $h_{i}$ are circularly-symmetric zero-mean complex Gaussian. Note that the receiver derived in this subsection is valid even for non-Rayleigh fading, provided that the received phases over the two channels are independent and uniform over $(0,2 \pi)$. When $b_{1} \neq b_{2}$ each element of $\mathbf{m}\left[b_{1}, b_{2}\right]$ is a circularlysymmetric zero-mean complex Gaussian and therefore has uniform phase. On the other hand, when $b_{1}=b_{2}$, one element is $h_{1}+h_{2}$, which is the sum of two circularly-symmetric zeromean complex Gaussians, while the other element is zero. Since the sum of two circularlysymmetric complex Gaussians is also a circularly-symmetric complex Gaussian, it follows that $h_{1}+h_{2}$ is a zero mean circularly-symmetric complex Gaussian and therefore its phase is uniform. Since the other element is zero, its phase is irrelevant and may be set to any arbitrary distribution, which is most conveniently chosen to be uniform. Thus, it follows that $\phi_{1}$ and $\phi_{2}$ are i.i.d. uniform. Therefore, the pdf conditioned on the magnitudes is

$$
\begin{aligned}
p\left(\mathbf{y}_{R} \mid \boldsymbol{\mu}\left[b_{1}, b_{2}\right]\right)= & \frac{1}{\pi N_{0}} \int_{0}^{2 \pi} \exp \left\{-\frac{\left|y_{1}-\mu_{1}\left[b_{1}, b_{2}\right] e^{j \phi_{1}}\right|^{2}}{N_{0}}\right\} d \phi_{1} \\
& \times \frac{1}{\pi N_{0}} \int_{0}^{2 \pi} \exp \left\{-\frac{\left|y_{2}-\mu_{2}\left[b_{1}, b_{2}\right] e^{j \phi_{2}}\right|^{2}}{N_{0}}\right\} d \phi_{2}
\end{aligned}
$$

where $\mu_{k}\left[b_{1}, b_{2}\right]$ is the $k^{\text {th }}$ element of $\boldsymbol{\mu}\left[b_{1}, b_{2}\right]$ and

$$
\frac{1}{2 \pi} \int_{0}^{2 \pi} \exp \left\{-\frac{\left|y_{k}-\mu_{k}\left[b_{1}, b_{2}\right] e^{j \phi_{k}}\right|^{2}}{N_{0}}\right\} d \phi_{k}=\exp \left\{-\frac{\left|y_{k}\right|^{2}+\left(\mu_{k}\left[b_{1}, b_{2}\right]\right)^{2}}{N_{0}}\right\} I_{0}\left(\frac{2\left|y_{k}\right| \mu_{k}\left[b_{1}, b_{2}\right]}{N_{0}}\right) \text {. }
$$

Substituting (2.18) into (2.17),

$$
p\left(\mathbf{y}_{R} \mid \boldsymbol{\mu}\left[b_{1}, b_{2}\right]\right)=\beta \prod_{k=1}^{2} \exp \left\{-\frac{\left(\mu_{k}\left[b_{1}, b_{2}\right]\right)^{2}}{N_{0}}\right\} I_{0}\left(\frac{2\left|y_{k}\right| \mu_{k}\left[b_{1}, b_{2}\right]}{N_{0}}\right)
$$


where

$$
\beta=\left(\frac{2}{N_{0}}\right)^{2} \exp \left\{-\left(\frac{\left|y_{1}\right|^{2}+\left|y_{2}\right|^{2}}{N_{0}}\right)\right\}
$$

which is common to all four $\left\{b_{1}, b_{2}\right\}$ and will therefore cancel in the LLR (2.10).

For each event $\left\{b_{1}, b_{2}\right\}$, substitute the $p\left(\mathbf{y}_{R} \mid \boldsymbol{\mu}\left[b_{1}, b_{2}\right]\right)$ given in (2.19) with the $\boldsymbol{\mu}\left[b_{1}, b_{2}\right]$ given by (2.14) and (2.15) as the corresponding $p\left(\mathbf{y}_{R} \mid\left\{b_{1}, b_{2}\right\}\right)$ in (2.10). This results in

$$
\begin{aligned}
\Lambda(b)= & \log \left[e^{-\alpha_{1}^{2} / N_{0}} I_{0}\left(\frac{2 \alpha_{1}\left|y_{1}\right|}{N_{0}}\right) e^{-\alpha_{2}^{2} / N_{0}} I_{0}\left(\frac{2 \alpha_{2}\left|y_{2}\right|}{N_{0}}\right)+e^{-\alpha_{2}^{2} / N_{0}} I_{0}\left(\frac{2 \alpha_{2}\left|y_{1}\right|}{N_{0}}\right) e^{-\alpha_{1}^{2} / N_{0}} I_{0}\left(\frac{2 \alpha_{1}\left|y_{2}\right|}{N_{0}}\right)\right] \\
& -\log \left[e^{-\alpha^{2} / N_{0}} I_{0}\left(\frac{2 \alpha\left|y_{1}\right|}{N_{0}}\right)+e^{-\alpha^{2} / N_{0}} I_{0}\left(\frac{2 \alpha\left|y_{2}\right|}{N_{0}}\right)\right] .
\end{aligned}
$$

Define $F(x)=\log \left[I_{0}(x)\right]$; then the LLR becomes

$$
\begin{aligned}
\Lambda(b)= & \frac{\alpha^{2}-\alpha_{1}^{2}-\alpha_{2}^{2}}{N_{0}}+\max *\left[F\left(\frac{2 \alpha_{1}\left|y_{1}\right|}{N_{0}}\right)+F\left(\frac{2 \alpha_{2}\left|y_{2}\right|}{N_{0}}\right), F\left(\frac{2 \alpha_{2}\left|y_{1}\right|}{N_{0}}\right)+F\left(\frac{2 \alpha_{1}\left|y_{2}\right|}{N_{0}}\right)\right] \\
& -\max *\left[F\left(\frac{2 \alpha\left|y_{1}\right|}{N_{0}}\right), F\left(\frac{2 \alpha\left|y_{2}\right|}{N_{0}}\right)\right] .
\end{aligned}
$$

In our simulations, we have discovered that the performance of the system is very sensitive to the numerical implementation of the $F(x)$ function. This function may be efficiently computed through the following piecewise polynomial fit, which returns precise answers over a wide range of the argument:

$$
F(x)=\log \left[I_{0}(x)\right]= \begin{cases}0.22594 x^{2}+0.012495 x-0.0011272 & 0<x \leq 1 \\ 0.12454 x^{2}+0.21758 x-0.10782 & 1<x \leq 2 \\ 0.028787 x^{2}+0.63126 x-0.56413 & 2<x \leq 5 \\ 0.003012 x^{2}+0.88523 x-1.2115 & 5<x \leq 15 \\ 0.00053203 x^{2}+0.95304 x-1.6829 & 15<x \leq 30 \\ 0.00013134 x^{2}+0.97674 x-2.0388 & 30<x \leq 60 \\ 0.9943 x-2.6446 & 60<x \leq 120 \\ 0.99722 x-3.0039 & 120<x \leq 500 \\ 0.99916 x-3.6114 & x>500 .\end{cases}
$$

As discussed in Section 2.4, it is possible to accurately estimate $\alpha_{1}$ and $\alpha_{2}$ in the considered block fading environment, provided the blocks are sufficiently long. However, it is 
not generally feasible to precisely estimate $\alpha$ because the phases $\theta_{1}$ and $\theta_{2}$ are varying on a symbol-by-symbol basis. Since $E\left[\cos \left(\theta_{2}-\theta_{1}\right)\right]=0$, a reasonable approximation when an estimate of $\alpha$ is not available is to use

$$
\alpha \approx \sqrt{\alpha_{1}^{2}+\alpha_{2}^{2}}
$$

Note that when such an approximation is used, the first term in (2.22) disappears.

\section{Noncoherent PNC Receiver without CSI}

Now suppose that besides not knowing the phases $\theta_{1}, \theta_{2}$, the relay receiver does not know the magnitude vectors $\boldsymbol{\mu}\left[b_{1}, b_{2}\right]$ either. Therefore, the relay must operate without any channel state information except for the average energies $\mathcal{E}_{1}, \mathcal{E}_{2}$ and the noise variance $N_{0}$. When the magnitudes $\boldsymbol{\mu}\left[b_{1}, b_{2}\right]$ are not known, then the conditional pdf is found by marginalizing (2.19) over the unknown magnitudes

$$
p\left(\mathbf{y}_{R} \mid\left\{b_{1}, b_{2}\right\}\right)=\int_{0}^{\infty} \int_{0}^{\infty} p\left(\mu_{1}, \mu_{2}\right) p\left(\mathbf{y}_{R} \mid \boldsymbol{\mu}\left[b_{1}, b_{2}\right]\right) d \mu_{1} d \mu_{2} .
$$

where $\mu_{1}$ and $\mu_{2}$ are the magnitudes of the first and second elements of $\boldsymbol{\mu}\left[b_{1}, b_{2}\right]$, respectively.

According to (2.14), when $b_{1} \neq b_{2}$, one of the $\mu_{k}=\alpha_{1}$ while the other $\mu_{k}=\alpha_{2}$. Since $\alpha_{1}$ and $\alpha_{2}$ are independent and each $\alpha_{i}$ is Rayleigh with energy $\mathcal{E}_{i}$, it follows that the joint pdf of $\mu_{1}$ and $\mu_{2}$ is

$$
p\left(\mu_{1}, \mu_{2}\right)=\left(\frac{2 \mu_{1}}{\mathcal{E}_{1}} \exp \left\{-\frac{\mu_{1}}{\mathcal{E}_{1}}\right\}\right)\left(\frac{2 \mu_{2}}{\mathcal{E}_{2}} \exp \left\{-\frac{\mu_{2}}{\mathcal{E}_{2}}\right\}\right), \mu_{1}, \mu_{2} \geq 0
$$

when $\left(b_{1}, b_{2}\right)=(0,1)$, and

$$
p\left(\mu_{1}, \mu_{2}\right)=\left(\frac{2 \mu_{1}}{\mathcal{E}_{2}} \exp \left\{-\frac{\mu_{1}}{\mathcal{E}_{2}}\right\}\right)\left(\frac{2 \mu_{2}}{\mathcal{E}_{1}} \exp \left\{-\frac{\mu_{2}}{\mathcal{E}_{1}}\right\}\right), \mu_{1}, \mu_{2} \geq 0 .
$$

when $\left(b_{1}, b_{2}\right)=(1,0)$. Substituting (2.26) and (2.19) into (2.25) yields

$$
p\left(\mathbf{y}_{R} \mid\left\{b_{1}=0, b_{2}=1\right\}\right)=\log \left[\left(\frac{1}{\mathcal{E}_{1} \mathcal{E}_{2}}\right)\left(\frac{1}{\mathcal{E}_{1}}+\frac{1}{N_{0}}\right)\left(\frac{1}{\mathcal{E}_{2}}+\frac{1}{N_{0}}\right)\right]^{-1}+\frac{\left|y_{1}\right|^{2}}{\frac{N_{0}^{2}}{\mathcal{E}_{1}}+N_{0}}+\frac{\left|y_{2}\right|^{2}}{\frac{N_{0}^{2}}{\mathcal{E}_{2}}+N_{0}} .
$$


Similarly, substituting (2.27) and (2.19) into (2.25) yields

$$
p\left(\mathbf{y}_{R} \mid\left\{b_{1}=1, b_{2}=0\right\}\right)=\log \left[\left(\frac{1}{\mathcal{E}_{1} \mathcal{E}_{2}}\right)\left(\frac{1}{\mathcal{E}_{1}}+\frac{1}{N_{0}}\right)\left(\frac{1}{\mathcal{E}_{2}}+\frac{1}{N_{0}}\right)\right]^{-1}+\frac{\left|y_{1}\right|^{2}}{\frac{N_{0}^{2}}{\mathcal{E}_{2}}+N_{0}}+\frac{\left|y_{2}\right|^{2}}{\frac{N_{0}^{2}}{\mathcal{E}_{1}}+N_{0}} .
$$

As indicated by (2.15), when $b_{1}=b_{2}$, one of the $\mu_{k}=\alpha$ while the other $\mu_{k}=0$. As discussed below (2.16), in a Rayleigh-fading environment, $h_{1}$ and $h_{2}$ are independent complex-valued, circularly-symmetric Gaussian variables, and therefore $h=h_{1}+h_{2}$ is also a complex-valued, circularly-symmetric Gaussian variable. It follows that $\alpha=|h|$ is Rayleigh with energy $\mathcal{E}_{1}+\mathcal{E}_{2}$, and thus the pdf of the nonzero $\mu_{k}$ is

$$
p\left(\mu_{k}\right)=\frac{2 \mu_{k}}{\mathcal{E}_{1}+\mathcal{E}_{2}} \exp \left\{-\frac{\mu_{k}}{\mathcal{E}_{1}+\mathcal{E}_{2}}\right\}, \mu_{k} \geq 0
$$

For the $\mu_{k}=0$, its pdf may be represented by an impulse at the origin, i.e. $p\left(\mu_{k}\right)=\delta\left(\mu_{k}\right)$. Substituting these pdfs with the appropriate $\boldsymbol{\mu}\left[b_{1}, b_{2}\right]$ into (2.25) yields

$$
p\left(\mathbf{y}_{R} \mid\left\{b_{1}, b_{2}\right\}\right)=\log \left[\left(\frac{1}{\mathcal{E}_{1}+\mathcal{E}_{2}}\right)\left(\frac{1}{\mathcal{E}_{1}+\mathcal{E}_{2}}+\frac{1}{N_{0}}\right)\right]^{-1}+\frac{\left|y_{i}\right|^{2}}{\frac{N_{0}^{2}}{\mathcal{E}_{1}+\mathcal{E}_{2}}+N_{0}}
$$

where $i=1$ when $\left(b_{1}, b_{2}\right)=(0,0)$ and $i=2$ when $\left(b_{1}, b_{2}\right)=(1,1)$.

Substituting (2.28) and (2.29) for the two $b_{1} \neq b_{2}$ and (2.31) for the two $b_{1}=b_{2}$ into (2.10) yields

$$
\begin{aligned}
\Lambda(b)= & \log \left[\frac{\xi_{1} \xi_{2}}{\xi N_{0}}\right]+\log \left[\exp \left\{-\frac{\left|y_{1}\right|^{2}}{\xi}-\frac{\left|y_{2}\right|^{2}}{N_{0}}\right\}+\exp \left\{-\frac{\left|y_{1}\right|^{2}}{N_{0}}-\frac{\left|y_{2}\right|^{2}}{\xi}\right\}\right] \\
& -\log \left[\exp \left\{-\frac{\left|y_{1}\right|^{2}}{\xi_{1}}-\frac{\left|y_{2}\right|^{2}}{\xi_{2}}\right\}+\exp \left\{-\frac{\left|y_{1}\right|^{2}}{\xi_{2}}-\frac{\left|y_{2}\right|^{2}}{\xi_{1}}\right\}\right]
\end{aligned}
$$

where

$$
\begin{aligned}
\xi_{1} & =\mathcal{E}_{1}+N_{0} \\
\xi_{2} & =\mathcal{E}_{2}+N_{0} \\
\xi & =\mathcal{E}_{1}+\mathcal{E}_{2}+N_{0} .
\end{aligned}
$$


In terms of $\max *(\cdot),(2.32)$ may be expressed as

$$
\begin{aligned}
\Lambda(b)= & \log \left[\frac{\xi_{1} \xi_{2}}{\xi N_{0}}\right]+\max *\left\{-\left(\frac{\left|y_{1}\right|^{2}}{\xi}+\frac{\left|y_{2}\right|^{2}}{N_{0}}\right),-\left(\frac{\left|y_{1}\right|^{2}}{N_{0}}+\frac{\left|y_{2}\right|^{2}}{\xi}\right)\right\} \\
& -\max *\left\{-\left(\frac{\left|y_{1}\right|^{2}}{\xi_{1}}+\frac{\left|y_{2}\right|^{2}}{\xi_{2}}\right),-\left(\frac{\left|y_{1}\right|^{2}}{\xi_{2}}+\frac{\left|y_{2}\right|^{2}}{\xi_{1}}\right)\right\} .
\end{aligned}
$$

\subsection{Channel Estimator}

The goal of the channel estimator is to estimate the values of the fading amplitudes $\alpha_{1}$ and $\alpha_{2}$ for a particular fading block. Let the fading amplitudes of a block be represented by the pair $\{A, B\}$, where $A \geq B$. Thus, $A=\max \left\{\alpha_{1}, \alpha_{2}\right\}$ and $B=\min \left\{\alpha_{1}, \alpha_{2}\right\}$. Note that in (2.22), exchanging $\alpha_{1}$ and $\alpha_{2}$ does not change the final expression. Therefore (2.22) is commutative in $\alpha_{1}$ and $\alpha_{2}$, and may be written as

$$
\begin{aligned}
\Lambda(b)= & \max *\left[F\left(\frac{2 A\left|y_{1}\right|}{N_{0}}\right)+F\left(\frac{2 B\left|y_{2}\right|}{N_{0}}\right), F\left(\frac{2 B\left|y_{1}\right|}{N_{0}}\right)+F\left(\frac{2 A\left|y_{2}\right|}{N_{0}}\right)\right] \\
& -\max *\left[F\left(\frac{2 \sqrt{A^{2}+B^{2}}\left|y_{1}\right|}{N_{0}}\right), F\left(\frac{2 \sqrt{A^{2}+B^{2}}\left|y_{2}\right|}{N_{0}}\right)\right]
\end{aligned}
$$

where we have used the approximation $\alpha \approx \sqrt{\alpha_{1}^{2}+\alpha_{2}^{2}}$.

\subsubsection{Fading Amplitude Estimator}

To estimate $A$ and $B$, first add the two elements of each $\mathbf{y}_{i, R}$ to obtain

$$
r_{i}=y_{i, 1}+y_{i, 2}=h_{i, 1}+h_{i, 2}+\underbrace{n_{i, 1}+n_{i, 2}}_{\nu_{i}}
$$

where $\nu_{i}$ is circularly-symmetric complex Gaussian noise with variance $2 N_{0}$, and $h_{i, k}$ is the channel coefficient between terminal $\mathcal{N}_{k}, k=\{1,2\}$, and the relay during the $i^{\text {th }}$ signaling interval. The signal $r_{i}$ is the sum of two frequency-modulated signals, and therefore the fading-amplitude estimation algorithm proposed by Hamkins in [60] may be used. To determine the values of $A$ and $B$, a system of two equations with two unknowns is required. 
The first equation, found by taking the expected value of $\left|r_{i}\right|^{2}$ under the assumption that the fading amplitudes are fixed for the block in question, is

$$
\begin{aligned}
E\left[\left|r_{i}\right|^{2}\right] & =E\left[\alpha_{1}^{2}+\alpha_{2}^{2}+2 \alpha_{1} \alpha_{2} \cos \left(\theta_{i, 2}-\theta_{i, 1}\right)\right]=E\left[\alpha_{1}^{2}+\alpha_{2}^{2}\right]=\alpha_{1}^{2}+\alpha_{2}^{2} \\
& =A^{2}+B^{2} .
\end{aligned}
$$

The second equation is found by conditioning on the event $\left\{|r|^{2}>A^{2}+B^{2}\right\}$, which is equivalent to $\left\{\cos \left(\theta_{i, 2}-\theta_{i, 1}\right)>0\right\}$ and has expected value [60]

$$
E\left[\left.|r|^{2}|| r\right|^{2}>A^{2}+B^{2}\right]=A^{2}+B^{2}+\frac{4 A B}{\pi} .
$$

Solving (2.37) and (2.38) for $A$ and $B$ yields

$$
\begin{aligned}
& A=\frac{1}{2}\left(\sqrt{X+\frac{\pi}{2}(Y-X)}+\sqrt{X+\frac{\pi}{2}(X-Y)}\right) \\
& B=\frac{1}{2}\left(\sqrt{X+\frac{\pi}{2}(Y-X)}-\sqrt{X+\frac{\pi}{2}(X-Y)}\right)
\end{aligned}
$$

where $X=E\left[|r|^{2}\right]$ and $Y=E\left[\left.|r|^{2}|| r\right|^{2}>A^{2}+B^{2}\right]$.

Since the expected values required for (2.39) are not known, they may be estimated by using corresponding statistical averages,

$$
\begin{aligned}
& \hat{X}=\frac{1}{N} \sum_{i=1}^{N}\left|r_{i}\right|^{2} \\
& \hat{Y}=\frac{2}{N} \sum_{i:\left|r_{i}\right|^{2}>\hat{X}}\left|r_{i}\right|^{2}
\end{aligned}
$$

where $N$ is the size of the fading block. As an alternative to summing over the $\left|r_{i}\right|^{2}>\hat{X}$, Hamkins proposes summing over those $\left|r_{i}\right|^{2}$ greater than the median value of $\left\{\left|r_{1}\right|^{2}, \ldots,\left|r_{N}\right|^{2}\right\}$ [60].

The estimator works by computing estimates $\hat{X}$ and $\hat{Y}$ using (2.40) and the $\left\{r_{1}, \ldots, r_{N}\right\}$ for the block. These estimates are used in place of $X$ and $Y$ in (2.39), which yields estimates $\hat{A}$ and $\hat{B}$ of $A$ and $B$, that are used in place of $A$ and $B$ in (2.35). 


\subsubsection{Transmission-Case Detection}

According to (2.36), the two elements of $\mathbf{y}_{i, R}$ are always added together. When $b_{1}=b_{2}$, only one tone is used, and the noise can be reduced if the receiver uses only the tone used and ignores the other tone. This requires that the receiver be able to detect whether the first tone, the second tone, or both tones were used, which may be implemented using a variation of the "no-CSI" receiver described in subsection 2.3.2. In [45] we contemplate an estimator that uses such a transmission-case detector. However, we found that the performance with and without the transmission-case detector were virtually identical and do not consider it further in this chapter. At best, proper use of the transmission-case detector reduces the noise variance from $2 N_{0}$ to $N_{0}$ during the symbol intervals that both nodes transmit the same tone. As will be seen in the numerical results, the estimator is resilient enough against noise that this reduction in noise variance is not meaningful and does not justify the additional complexity.

\subsubsection{Amplitude Estimation for Single-Transmitter Links}

During the broadcast stage, there is only a single transmission, and the dual-amplitude estimator described in subsection 2.4.1 is not necessary. Similarly, the estimator is not needed by the LNC system during the MA stage since the two transmissions are over orthogonal channels. To estimate the fading amplitudes for the links involving only a single transmitter and receiver, the simple averaging technique given by (29) in [61] is used, which is described as follows. Consider the $i^{\text {th }}$ signaling interval during the $\ell^{\text {th }}$ fading block. Given transmission of tone $k$, in the absence of noise, the $k^{\text {th }}$ matched-filter output at the receiver is $y_{k, i}=\alpha e^{j \theta_{i}}$, and has magnitude $\left|y_{k, i}\right|=\alpha$. All other matched-filter outputs in the $i^{\text {th }}$ signaling interval are 0 . An estimate could be formed by taking the maximum $\left|y_{k, i}\right|$ over any column of $\mathbf{Y}_{R}^{(\ell)}$. In the presence of noise, an estimate of $\alpha$ can be formed by averaging across all columns of the fading block

$$
\hat{\alpha}=\frac{1}{N} \sum_{i=1}^{N} \max _{k}\left|y_{k, i}\right|
$$




\subsection{Simulation Study}

This section presents simulated performance results for the relay receiver described in Section 2.3. The simulated link model is as described in Section 2.2, with specific simulation parameters given in the following subsections. The goal of the simulations is to compare the performance of comparable DNC and LNC systems and to assess the robustness of the channel estimator proposed in 2.4. Because the relay-broadcast stage of the DNC and LNC systems operate in exactly the same manner and have the same performance, we only focus on the performance of the MA stage.

\subsubsection{Uncoded Performance with Perfect Channel Estimates}

We initially consider a system that does not use an outer error-correcting code, and thus $\mathbf{b}_{i}=\mathbf{u}_{i}, i=1,2$. We compare the performance of the LNC and DNC systems. With the LNC system, the two nodes transmit their messages in orthogonal time slots and the relay receiver first generates the individual LLR's during each time slot using either (2.7) or (2.8), and then the two LLR's are combined using (2.9). When there is no outer error-correcting code, performance using (2.7) is approximately the same as that using (2.8). A bit error is declared at the relay whenever a hard decision using (2.9) results in an erroneous decision on the corresponding bit of the network codeword b. Such an error will usually occur if one of the two bits $b_{1}, b_{2}$ is received incorrectly, and therefore the error rate of the LNC system is approximately $P_{b} \approx 2 p(1-p)$ where $p$ is the bit error rate of noncoherent binary FSK modulation [18].

With the DNC system, the two nodes transmit simultaneously, and the relay receiver computes the LLR using (2.22) when the magnitudes $\boldsymbol{\mu}\left[b_{1}, b_{2}\right]$ are known or (2.34) when they are not. A hard decision is made on the LLR and a bit error is declared if the estimate

of the corresponding network codeword bit $b$ is incorrect. We assume that the channel estimates are perfect, and since there is no error-correction coding, the size of the fading block is irrelevant. 


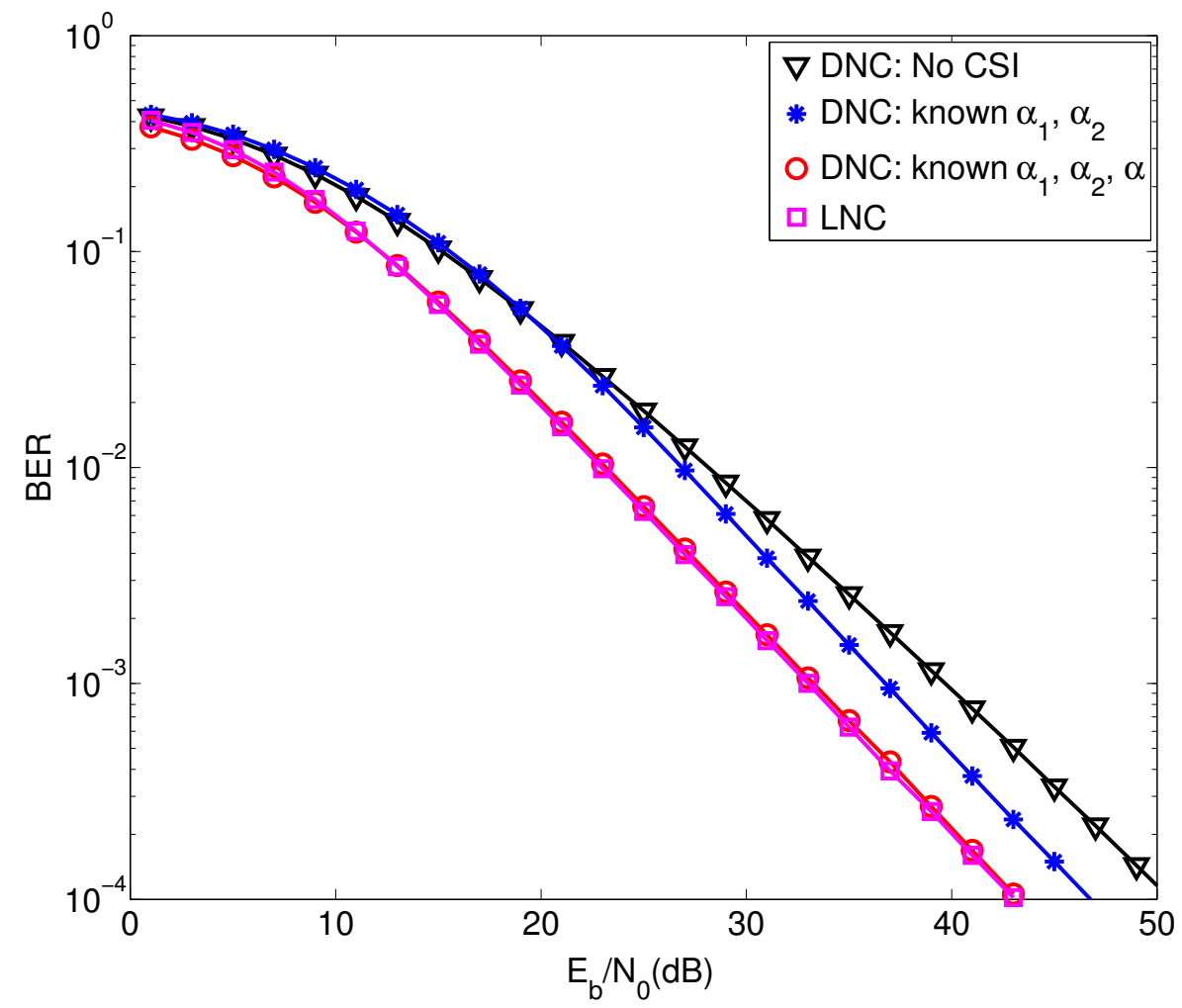

Figure 2.2: Bit error rate at the relay in Rayleigh fading when DNC and LNC is used and $\mathcal{E}_{2}=\mathcal{E}_{1}$. Depending on the amount of channel state information that is available, the PNC system will use one of three different relay receivers.

Initially, we set the average received energy to be the same over both channels, i.e. $\mathcal{E}_{2}=\mathcal{E}_{1}=\mathcal{E}_{s}=\mathcal{E}_{b}$. Fig. 2.2 shows the performance of the LNC and DNC systems in Rayleigh fading with equal energy signals. As anticipated, the LNC system offers the best performance, which is approximately $3 \mathrm{~dB}$ worse than a standard binary CPFSK system with noncoherent detection (the loss relative to conventional CPFSK is due to the fact that both bits must usually be received correctly). Three curves for the DNC system are shown in Fig. 2.2, corresponding to receivers that exploit different amounts of available channel state information. The best performance is achieved using a receiver implemented with (2.22), which requires knowledge of $\alpha_{1}, \alpha_{2}$, and $\alpha$. The performance of the DNC system implemented with (2.22) is only about $0.25 \mathrm{~dB}$ worse than that of the LNC system. The worst performance is achieved using a receiver implemented using (2.34), which does not 


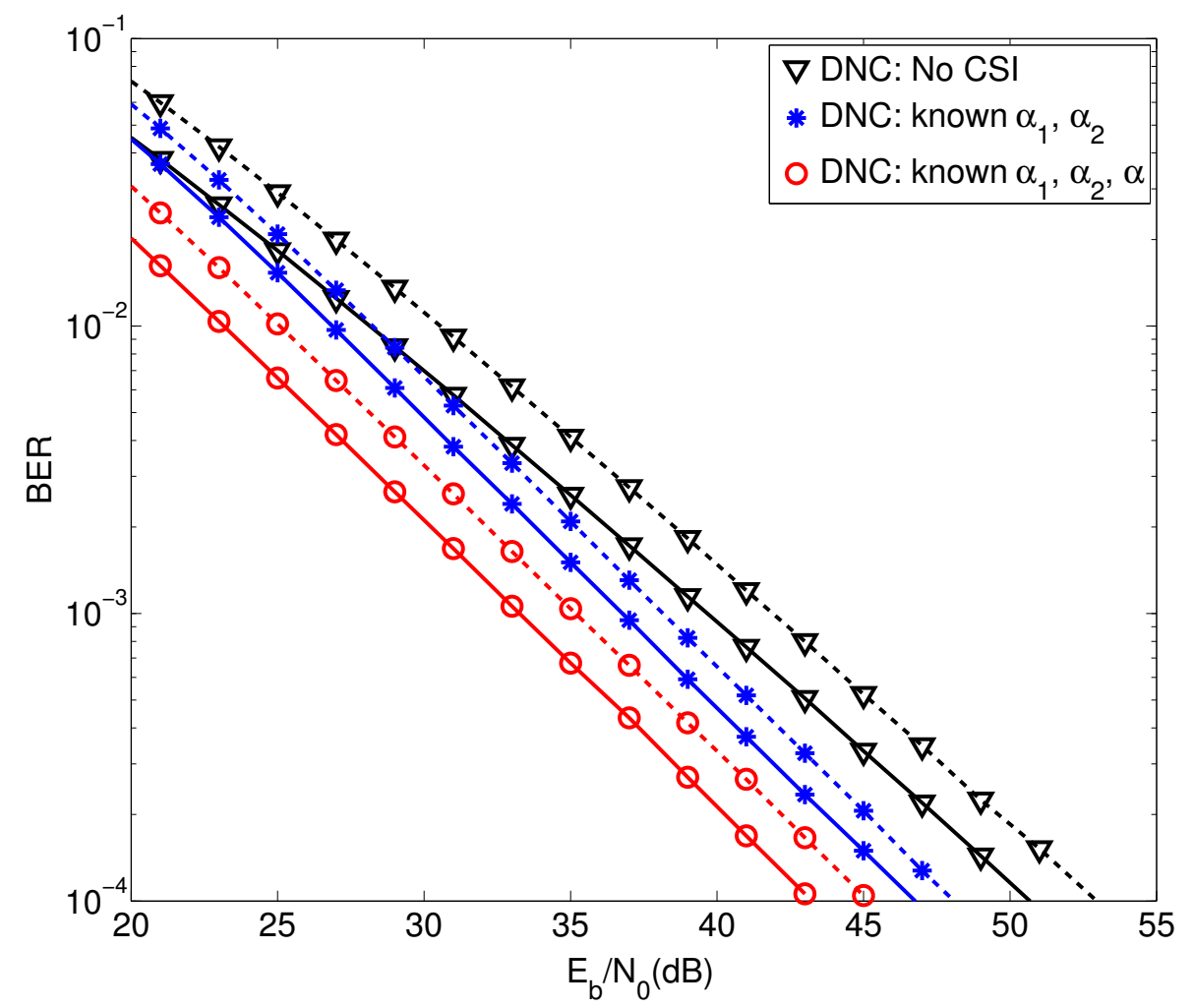

Figure 2.3: Bit error rate at the relay in Rayleigh fading when DNC is used with three different receivers and either $\mathcal{E}_{2}=\mathcal{E}_{1}$ (solid line) or $\mathcal{E}_{2}=4 \mathcal{E}_{1}$ (dashed line).

require knowledge of the fading amplitudes. The loss due to using (2.34) instead of (2.22) is about $10 \mathrm{~dB}$, indicating that estimating the fading amplitudes at the relay is beneficial.

While it may be feasible to estimate $\alpha_{1}$ and $\alpha_{2}$, estimating $\alpha$ may prove to be more difficult because it will depend on not only the individual fading amplitudes, but also on the phase difference between the two channels. Since the phase difference might change more quickly than the individual amplitudes, it might not be practical to estimate $\alpha$. If that is the case, then the approximation given by (2.24) can be used in place of the actual value of $\alpha$. The performance using this technique is also shown in Fig. 2.2 and shows a loss of about $3 \mathrm{~dB}$ with respect to the known- $\boldsymbol{\mu}\left[b_{1}, b_{2}\right]$ system, which requires knowledge of $\alpha$.

The performance of DNC is sensitive to the balance of power received over the two channels. Performance is best when $\mathcal{E}_{1}=\mathcal{E}_{2}$. In order to evaluate how robust the DNC relay 


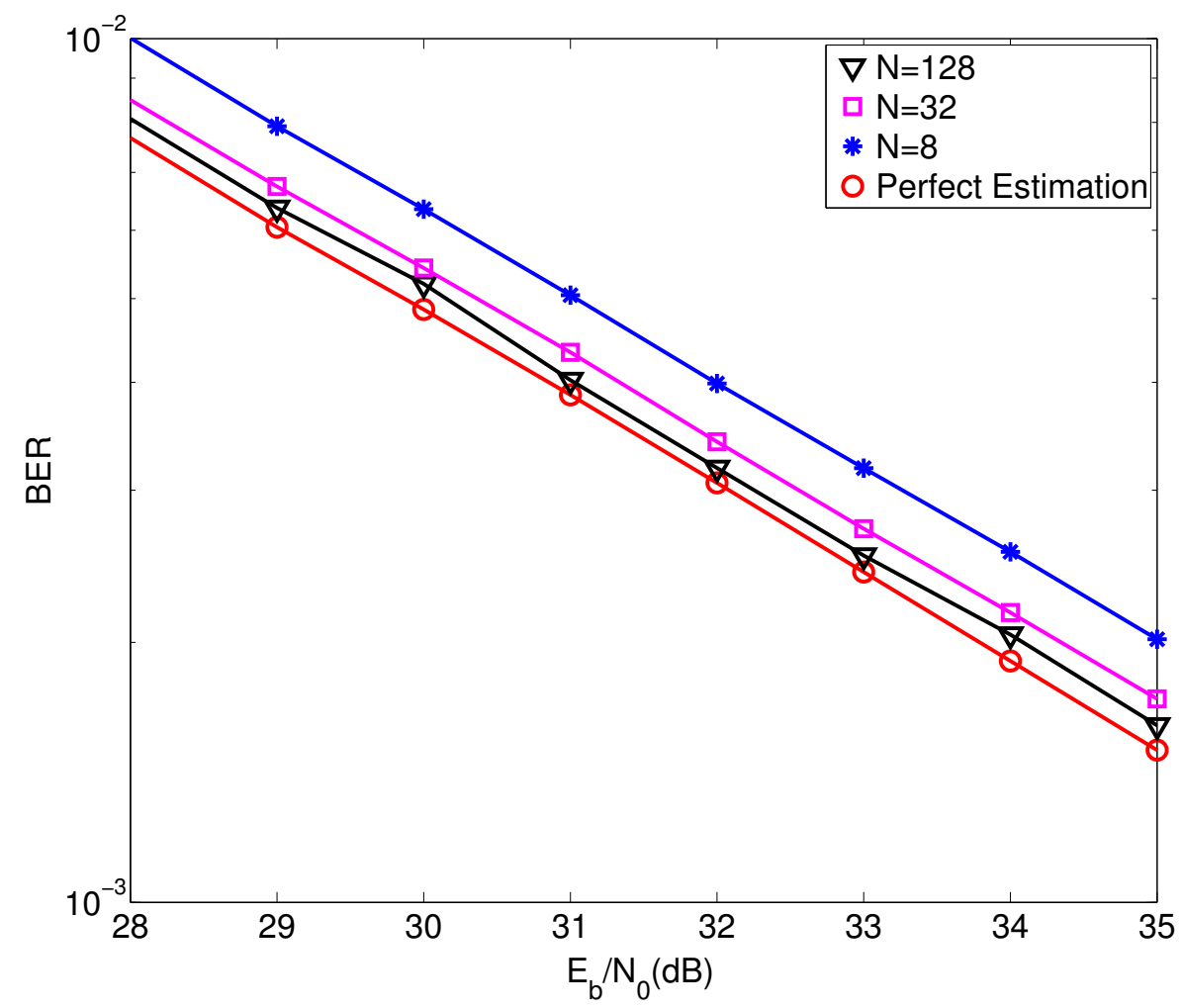

Figure 2.4: Influence of fading-block length $N$ on uncoded DNC error-rate performance at the relay. In addition to curves for three values of $N$, a curve is shown indicating the performance with perfect fading-amplitude knowledge.

receivers are to an imbalance of power, the simulations were repeated with $\mathcal{E}_{2}=4 \mathcal{E}_{1}$, while keeping $\mathcal{E}_{b}=\mathcal{E}_{s}=\left(\mathcal{E}_{1}+\mathcal{E}_{2}\right) / 2$. These results are shown in Fig. 2.3 for the three receiver formulations that were considered in the previous figure. When the power is imbalanced in this way, there is a loss of about $2 \mathrm{~dB}$. However, the loss is the same for all three receiver implementations, suggesting that they are robust to an imbalance of power.

\subsubsection{Uncoded Performance with Channel Estimation}

We now consider the influence of channel estimation, but still assume that the system does not use error-correction coding. In the simulations, the information frames generated at the end nodes contain $K=2048$ bits per frame. The fading blocks are length $N=\{8,32,128\}$ 
symbols per block. The DNC relay implements (2.22) with the approximation given by (2.24) and then makes a hard decision on each information bit.

The bit error-rate performance of the uncoded system is shown in Fig. 2.4. The performance is shown with the estimator using the three block sizes $N=\{8,32,128\}$ as well as for the case of perfect estimates of $\alpha_{1}$ and $\alpha_{2}$. A narrow range of error rates is shown to better highlight the differences in performance. In general, smaller fading blocks lead to a less accurate estimation of the fading amplitudes, as the number of samples available for estimation decreases. Moving from block size $N=128$ to 32 worsens performance by roughly $0.25 \mathrm{~dB}$, and from $N=32$ to 8 by $0.75 \mathrm{~dB}$.

\subsubsection{Performance with an Outer turbo Code}

Now consider a system that uses an outer turbo code. The terminals each encode length $K=1229$ information sequences into length $L=2048$ codewords, using a rate $r_{1} \approx 0.6$ UMTS turbo code [62]. The relay performs turbo decoding using the codeword LLR's computed by (2.22) with the approximation for $\alpha$ given by (2.24). The fading-block lengths simulated are $N=\{8,16,32,64,128\}$ symbols per block.

The error performance of the coded system is shown in Fig. 2.5, both with perfect channel estimates and with estimated fading amplitudes. A good tradeoff between diversity and estimation accuracy is achieved for block sizes $N=16$ and $N=32$, which exhibit the best performance of all systems that must estimate the fading amplitudes. For $N<16$ performance degrades due to the lack of enough observations per block for accurate channel estimates, while for $N>32$ performance degrades due to the reduction in time diversity.

Fig. 2.6 shows the SNR required to reach an error rate of $10^{-4}$ at the relay as a function of the block length $N$. In each case, information is coded with the same $(2048,1229)$ turbo code used for Fig. 2.5. Curves for three systems are shown: The noncoherent receiver with known $\left\{\alpha_{1}, \alpha_{2}\right\}$, the noncoherent receiver with estimated $\left\{\alpha_{1}, \alpha_{2}\right\}$, and the noncoherent receiver that does not use CSI. When $\left\{\alpha_{1}, \alpha_{2}\right\}$ are not estimated, performance improves with decreasing $N$ because of the increased number of blocks per codeword, which increases 


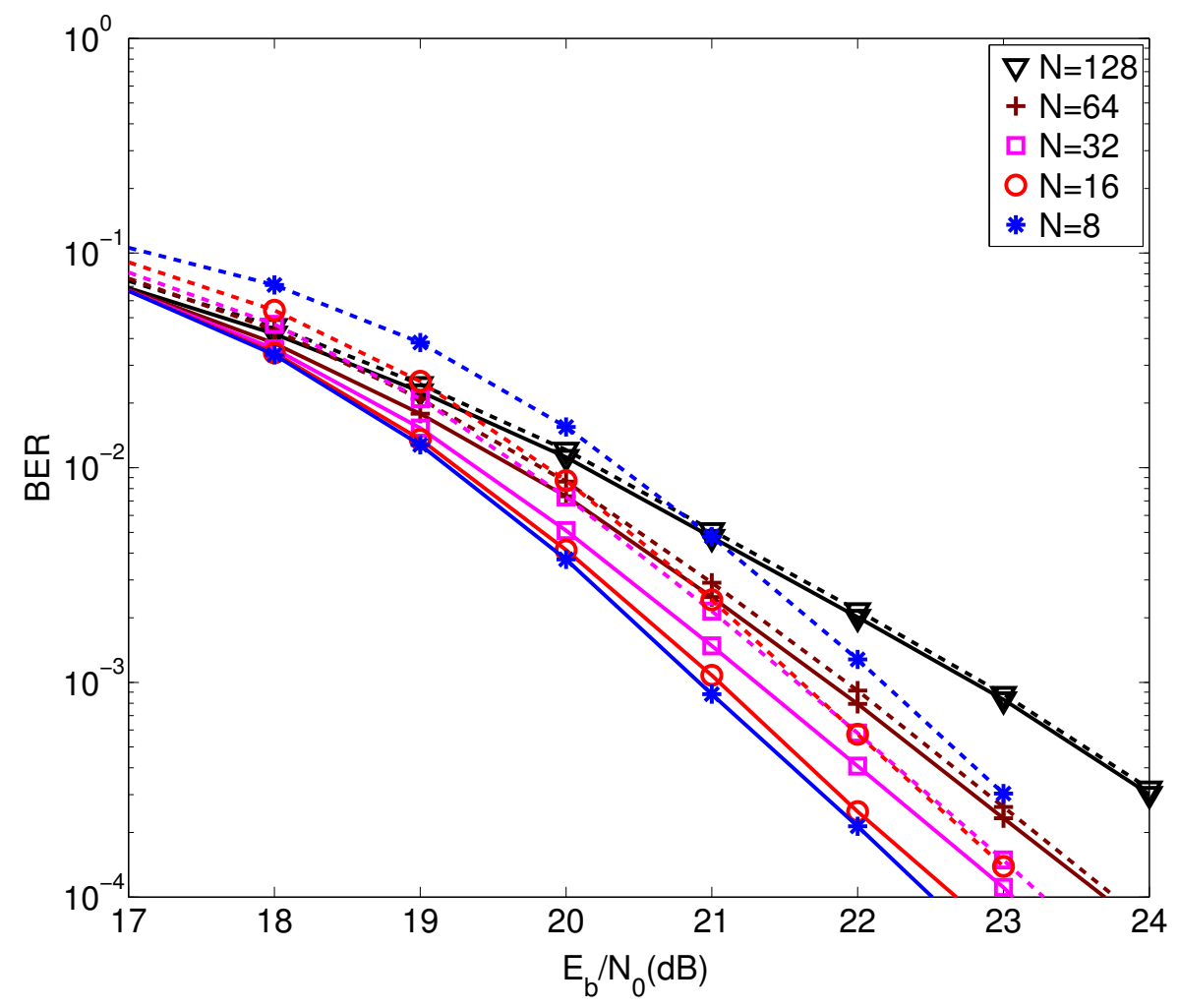

Figure 2.5: Influence of fading-block length $N$ on turbo-coded DNC error-rate performance at the relay. Two curves are shown for each value of $N=\{8,16,32,64,128\}$. Solid curves denote perfect fading-amplitude knowledge. Dashed curves denote estimated fading amplitudes.

the time diversity. However, when $\left\{\alpha_{1}, \alpha_{2}\right\}$ are estimated, the performance gets worse when the block size is smaller than $N=16$. The performance gap between the known-CSI and no-CSI receiver formulations widens with increasing block length.

An error-rate performance comparison between DNC and LNC is shown in Fig. 2.7. Both systems use the same $(2048,1229)$ turbo code. The LNC system outperforms the DNC system by margins ranging between 4 and $6 \mathrm{~dB}$.

While the LNC system is more energy efficient than the DNC system when the samerate turbo code is used, the throughput of the LNC system is worse than that of the DNC system because the two terminals must transmit in orthogonal time slots. The loss in energy efficiency from using DNC versus LNC can be recovered by having the source terminals use a lower-rate turbo code. Consider the performance comparison shown in Fig. 2.8 for block 


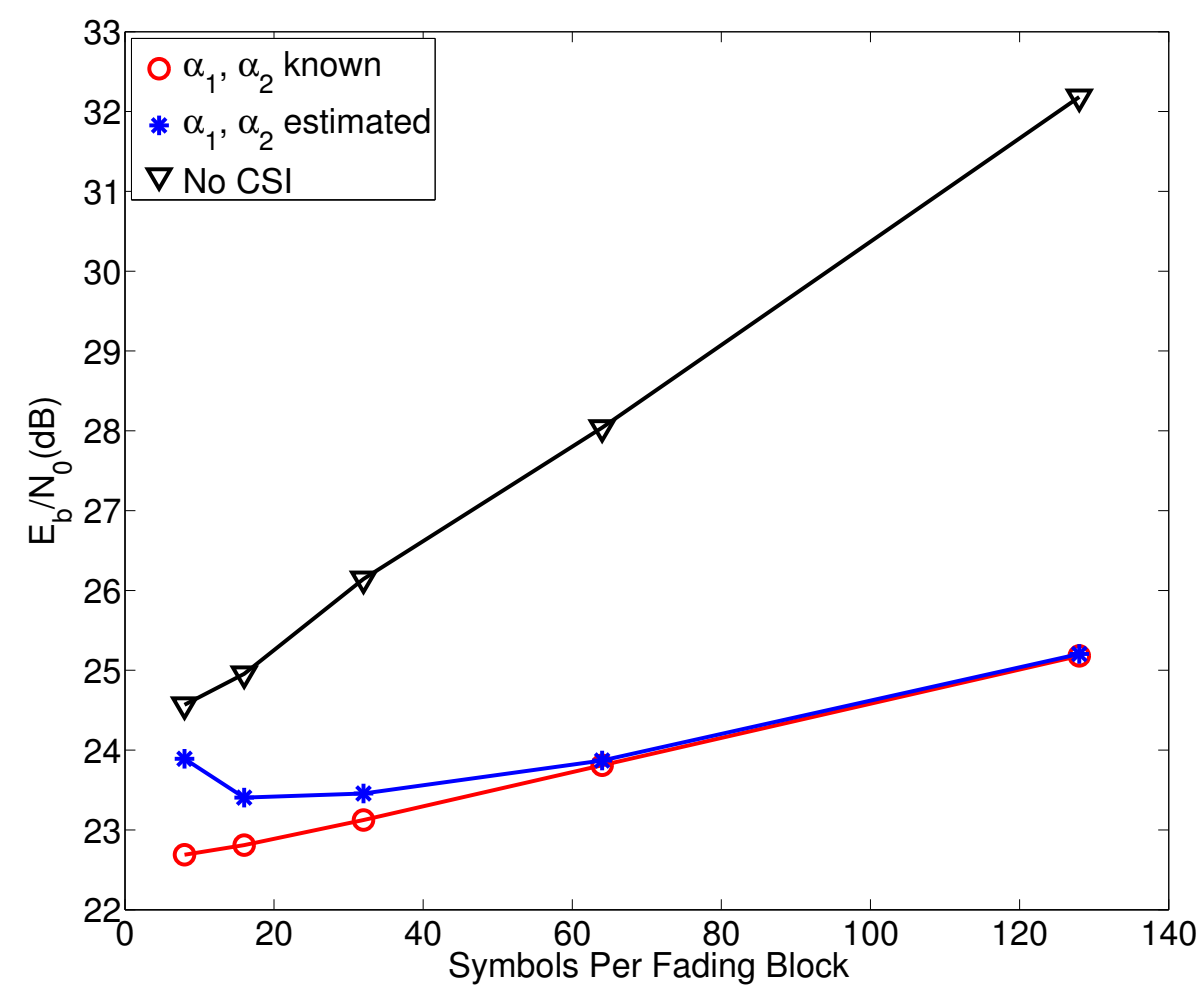

Figure 2.6: SNR required to reach a bit error rate of $10^{-4}$ at the relay as a function of fading-block length. Three systems are shown: The noncoherent receiver with known and estimated $\left\{\alpha_{1}, \alpha_{2}\right\}$ and with no CSI. All systems use a turbo code with rate 1229/2048.

size $N=32$. At $E_{b} / N_{0} \approx 24 \mathrm{~dB}, \mathrm{DNC}$ using a rate $r_{1}=4500 / 6400$ code matches the error-rate performance of LNC using a rate $r_{1}=4500 / 5056$ code. Because the two terminals transmit at the same time, the end-to-end throughput of DNC is higher than that of LNC, even though the DNC terminals transmit to the relay with a lower-rate channel code.

To illustrate the throughput improvement of DNC over LNC, consider the following transmission schedule for the two systems. Assume the source terminals use rate $r_{1}=4500 / 6400$ in DNC, and $r_{1}=4500 / 5056$ in LNC. Assume operation at $E_{b} / N_{0}=24 \mathrm{~dB}$, yielding approximately equal relay error-rate performance. Further, assume that both systems use code rate $r_{2}=4500 / 5056$ for relay broadcast, yielding approximately equal end-to-end performance. DNC requires 6400 channel uses for transmission to the relay versus $2 \times 5056=10112$ for LNC. Both systems require 5056 channel uses for relay broadcast. The throughput for DNC 


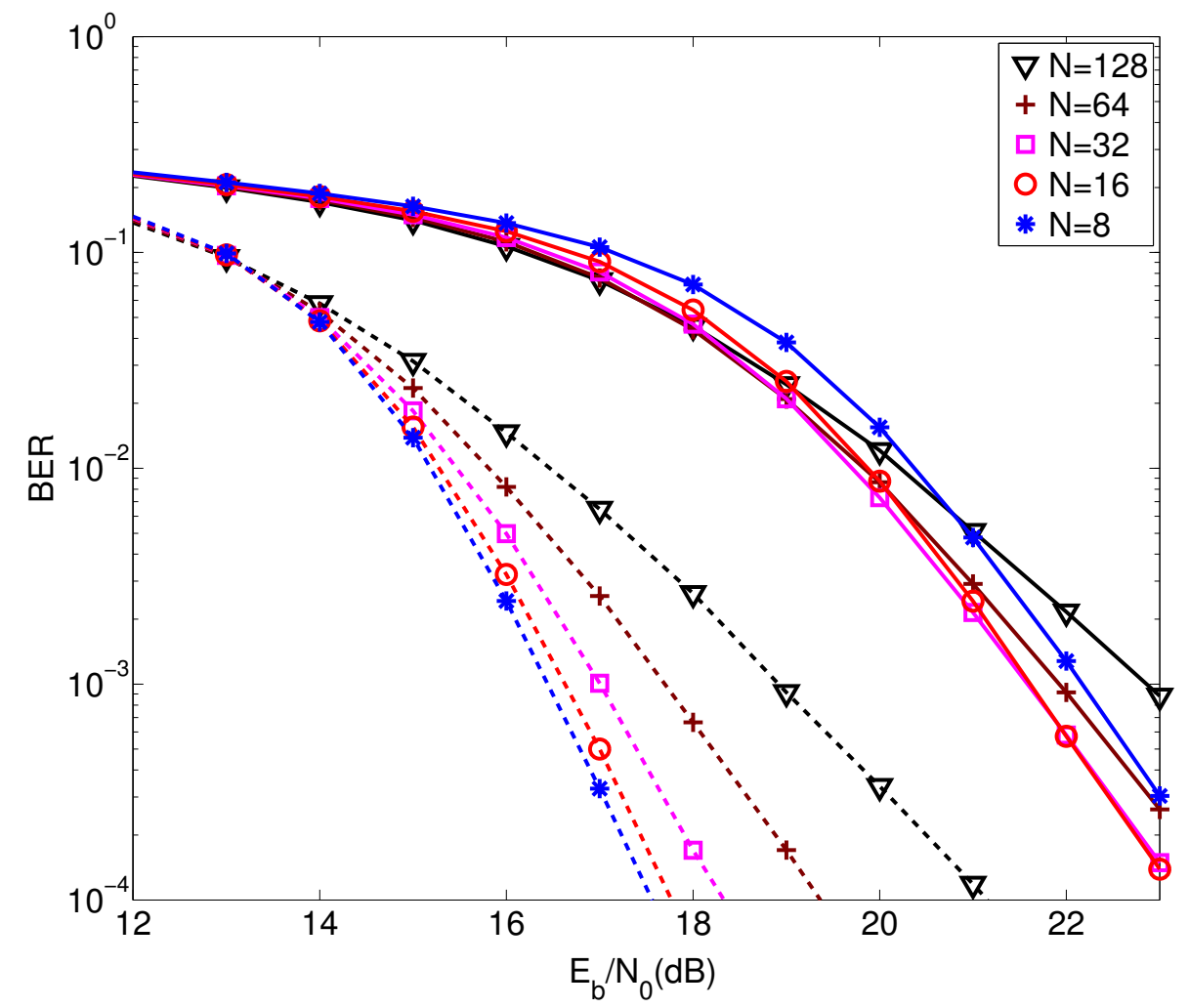

Figure 2.7: Comparison of error-rate performance between the turbo-coded DNC and LNC systems at the relay. The solid lines denote DNC, while the dashed lines denote LNC.

is thus $T^{(D N C)}=9000 /(6400+5056)=9000 / 11,456$ bits per channel use, and for LNC $T^{(L N C)}=9000 /(3 \times 5056)=9000 / 15,168$ bits per channel use. The percentage throughput increase of DNC over LNC is thus $\left(T^{(D N C)} / T^{(L N C)}-1\right) \times 100 \approx 32.4 \%$.

\subsection{Conclusion}

A throughput-improving technique for relaying in the two-way relay network, digital network coding, is refined for practical operation. The system operates noncoherently, providing advantages over coherent operation: there are no requirements for perfect power control, phase synchronism, or estimates of carrier-phase offset.

A computationally simple technique for estimating fading amplitudes at the relay is implemented. Error-rate performance in the noncoherent Rayleigh block-fading channel at 


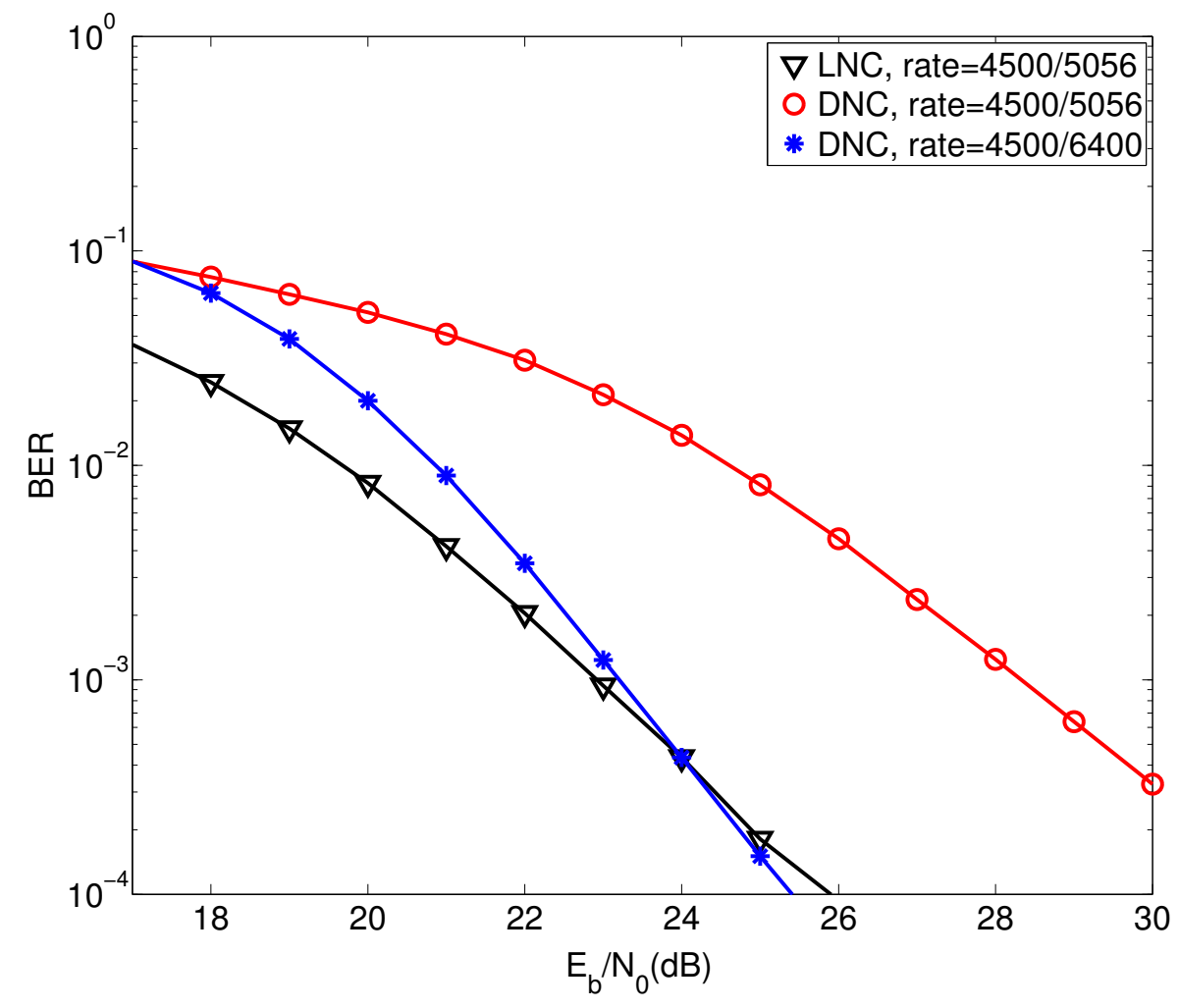

Figure 2.8: Comparison of the performance of turbo-coded DNC and LNC at the relay with block size $N=32$. For the DNC system, two code rates are shown, with the lower rate code offering comparable performance to the LNC system.

several block sizes is presented. The system is simulated with and without an outer errorcorrecting code. The coded error-rate performance of the system using estimation differs from that with ideal estimates by margins between $0.7-1.5 \mathrm{~dB}$.

When the same-rate turbo code is used, digital network coding has a higher throughput but lower energy-efficiency than link-layer network coding. The energy loss of DNC can be recovered by using a lower-rate turbo code during the MA stage. Even when the loss of spectral efficiency due to the lower-rate turbo code is taken into account, the DNC system is able to achieve a higher throughput than LNC at the same energy-efficiency. In the particular example presented in this chapter, the DNC system is capable of achieving throughputs that are $32.4 \%$ larger than that of the equivalent LNC system, while operating at the same energy efficiency. 


\section{Chapter 3}

\section{Iterative Noncoherent M-ary FSK System for DNC}

The previous chapter developed a soft-output binary FSK demodulator for the twoway relay channel multiple-access phase, operated with digital network coding. In this chapter, we extend the demodulator formulation from binary to M-ary and incorporate feedback from the channel decoder to refine symbol likelihood estimates. These extensions improve the energy efficiency and capacity of the system. The demodulator bit-error rate performance is simulated with and without LDPC channel coding. Capacity in both the multiple-access phase and end-to-end is analyzed, simulated, and used as a means to evaluate the performance of coded simulation.

\subsection{Introduction}

A very general approach for combining binary channel coding and $M$-ary modulation is bit-interleaved coded modulation (BICM) [34]. A binary channel codeword is generated at the source, interleaved, and passed to an $M$-ary modulator, which maps codeword bits to symbols for transmission over the channel. The receiver demodulates the symbols, producing soft estimates of each bit. A binary soft-input channel decoding scheme is applied to the soft estimates. The performance of BICM can be improved by feeding back information from 
the channel decoder to the demodulator, making use of iterative detection, which is called BICM with iterative decoding (BICM-ID) [35]. Iterative detection exhibits performance approaching the coded-modulation (CM) capacity. CM capacity provides an upper limit on the performance of joint demodulation and channel coding schemes [34], which BICM-ID approximates [35]. The performance of BICM-ID combined with PNC has been shown to closely approach CM capacity [63].

Recently, attention has been paid in the literature to combining BICM-ID with DNC link-by-link channel coding. In [64], BICM-ID is combined with a nonbinary convolutional channel code at the relay to perform channel decoding prior to network coding. A demodulator and decoder structure using quadrature amplitude modulation (QAM) and convolutional coding with BICM-ID is developed in [65]. Extrinsic information transfer (EXIT) analysis is applied to optimize the constellation mapping for a BICM-ID system considering quadrature phase-shift keying (QPSK) and repeat-accumulate channel coding in [37]. One of our key contributions in the present work is the development of a noncoherent relay demodulator suitable for BICM-ID and M-FSK modulation.

While DNC enables higher transmission rates than conventional three-step network coding techniques where the end nodes transmit to the relay in separate time slots, the deliberate interference encountered by the relay during the MA phase complicates receiver design. In order to motivate and justify the DNC approach, throughout this work we compare performance against LNC. Specifically, the capacity and error rate performance of both techniques are analyzed, simulated and compared.

The previous chapter developed a soft-output noncoherent binary FSK demodulator for the TWRC relay that can operate under several cases of available channel state information. With regards to our peer-reviewed work, the binary demodulator formulation presented in the the previous chapter was extended to M-ary in [29], and further developed to an M-ary demodulator capable of feeding back information from decoder to demodulator (BICM-ID) in [30]. The capacity of the TWRC using DNC and LNC was analyzed in [47]. The focus of the present chapter is to gather and present the aformentioned results. 
The specific contributions of this chapter are:

1. We formulate a soft-output DNC relay demodulator for M-ary FSK. It is capable of operating noncoherently under several cases of channel state information.

2. We develop a BICM-ID relay receiver for a channel-coded uplink. In the receiver, information is fed back from the decoder to the demodulator, refining the symbol likelihood estimates, and thereby improving bit error rate performance.

3. We perform a capacity analysis of the noncoherent DNC uplink and downlink, which we demonstrate is a good predictor for the performance of the system when using optimized codes.

4. DNC performance is compared against LNC throughout, quantifying the circumstances under which each technique exhibits the best performance.

The rest of this chapter is organized as follows. Section 3.2 develops the system model. Section 3.3 formulates the DNC relay demodulator. Section 3.4 analyzes the capacity of the TWRC for both DNC and LNC. Section 3.5 presents simulated bit error rate performance for DNC and LNC, Section 3.6 provides concluding remarks.

\subsection{System Model}

This section presents the system model details specific to the contribution provided in this chapter. Transmission by the end-nodes in the multiple access phase follows the model described in the introductory transmission model section, 1.3.1, and the assumed channel is described in the introductory channel model section, 1.3.2. LDPC channel coding is considered. Detection of the network-coded bits during relay reception is described. The subscript $P$ denotes a parameter having value that depends on the network coding scheme, for example, $r_{P}$ denotes a channel code rate taking value $r_{D}$ for DNC and $r_{L}$ for LNC. The system model for the DNC MA phase is depicted graphically in Fig. 3.1. 


\subsubsection{Relay Reception}

The goal of the relay receiver is to detect the network-coded combination of information bits transmitted by the end nodes, $\mathbf{u}=\mathbf{u}_{1} \oplus \mathbf{u}_{2}$, where $\oplus$ denotes the exclusive-or operation. The relay receiver takes the received frames as input. Considering LNC, the relay demodulates and decodes the codewords transmitted by each end node separately using conventional point-to-point techniques, yielding estimates of $\mathbf{u}_{1}$ and $\mathbf{u}_{2}$ that are combined by exclusive-or to detect the network-coded combination of bits. For DNC, the relay demodulator computes the probabilities of receiving the network-coded combination of bits transmitted by the end nodes

$$
\mathbf{b}=\mathbf{b}_{1} \oplus \mathbf{b}_{2}=\left[b_{0}\left(\mathbf{x}_{k, 1}\right) \oplus b_{0}\left(\mathbf{x}_{k, 2}\right) \ldots b_{\mu-1}\left(\mathbf{x}_{k, 1}\right) \oplus b_{\mu-1}\left(\mathbf{x}_{k, 2}\right)\right] \quad \forall k \in\left\{1, \ldots, L_{P}\right\}
$$

where $b_{m}\left(\mathbf{x}_{k, i}\right)$ denotes the $m$-th bit mapped to the $k$-th symbol transmitted by end node $\mathcal{N}_{i}$. Since the LDPC code is a systematic linear code, the modulo-2 sum of transmitted bits forms a code from the codebooks used by the end nodes, thus, the channel decoding operation yields a decision on the network-coded message bits $\mathbf{u}$.

In order to fairly compare DNC and LNC, the duration of the MA phase, as well as the information length $K$, are assumed the same for both. For DNC, both end nodes transmit during the entire MA phase. For LNC, the MA phase is divided evenly between the two end nodes. To transmit the same number of network-coded bits as DNC during the MA phase, each end node in LNC must transmit at twice the rate as the DNC end nodes. Define the $M A$ rate as total number of network-coded information bits received at the relay during the MA phase divided by the total number of bits transmitted during the MA phase $r_{M}=K / N$, where $N$ is the total number of bits transmitted during the multiple access phase, thus, $r_{M}=r_{D}=r_{L} / 2$. The total number of symbol time slots during the MA phase is $L=N / \mu$. The frame structure for the MA phase is shown in Fig. 3.2.

The demodulator takes as input one of the matrices of received symbols $\mathbf{Y}_{R}, \mathbf{Y}_{1, R}$ or $\mathbf{Y}_{2, R}$ and a priori probability (APP) information $\mathbf{v}$ and produces a posteriori information $\mathbf{z}$. The a posteriori information is deinterleaved to produce $\mathbf{z}^{\prime}=\mathbf{z} \Pi^{-1}$ and passed to the decoder. 


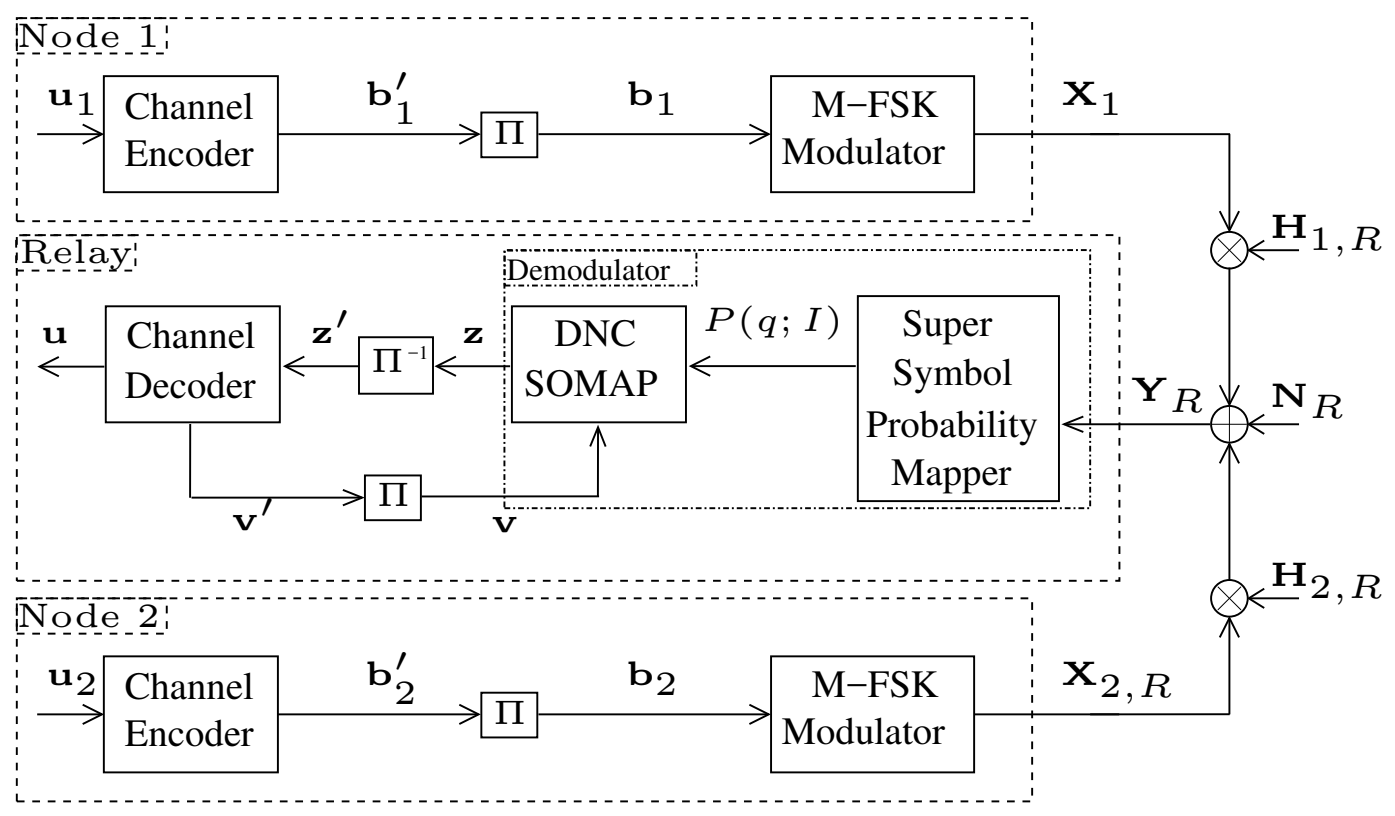

Figure 3.1: System Model for DNC two-way relay channel multiple-access phase with iterative decoding.

The decoder refines the estimate of $\mathbf{z}^{\prime}$, producing a posteriori information $\mathbf{v}_{o}^{\prime}$. The decoder input is subtracted from the decoder output to produce extrinsic information $\mathbf{v}_{e}^{\prime}=\mathbf{v}_{o}^{\prime}-\mathbf{z}^{\prime}$ which is interleaved to produce $\mathbf{v}_{e}=\mathbf{v}_{e}^{\prime} \boldsymbol{\Pi}$ and returned to the demodulator. The decoder output becomes the demodulator a priori input $\mathbf{v}_{e}=\mathbf{v}_{a}$.

The average symbol signal-to-noise ratio $\mathcal{E}_{i} / N_{0}$ transmitted by each end node is known at the demodulator. The demodulator may operate under several cases of channel state information (CSI): the case in which the gain are completely known (full CSI), the case in which only the fading amplitudes $\alpha_{k, i}$ are known (partial CSI), and the case in which no information about the gains is known (no CSI).

\subsubsection{Broadcast Phase}

The relay encodes and modulates the network-coded message bits $\mathbf{u}$ and broadcasts to the end nodes. The signal traverses two independent fading channels, and the end nodes receive independently faded versions of the message bits corrupted by white noise, $\hat{\mathbf{u}}$ at $\mathcal{N}_{1}$ and $\tilde{\mathbf{u}}$ at $\mathcal{N}_{2}$. The end nodes demodulate and decode the signal received from the relay to 


\begin{tabular}{|c|c|c|c|c|c|c|c|}
\hline \multirow{2}{*}{$\mathrm{DNC}$} & $\mathbf{x}_{1,1}$ & $\mathbf{x}_{2,1}$ & \multicolumn{4}{|c|}{$\cdots$} & $\mathbf{x}_{L, 1}$ \\
\hline & $\mathbf{x}_{1,2}$ & $\mathbf{x}_{2,2}$ & \multicolumn{4}{|c|}{$\cdots$} & $\mathbf{x}_{L, 2}$ \\
\hline \multirow{2}{*}{ LNC } & $\mathbf{x}_{1,1}$ & $\mathbf{x}_{2,1}$ & $\ldots \mathbf{x}_{\frac{L}{2}, 1}$ & & & & \\
\hline & & & & $\mathbf{x}_{1,2}$ & $\mathbf{x}_{2,2}$ & $\cdots$ & $\mathbf{x}_{\frac{L}{2}, 2}$ \\
\hline
\end{tabular}

Figure 3.2: Frame structure for digital and link-layer network coding (DNC and LNC) during the TWRC multiple-access phase. For DNC, the end nodes each transmit $L$ symbols simultaneously. For LNC, each end node transmits $L / 2$ symbols in separate time slots.

form estimates of $\mathbf{u}$. Each end node estimates the information bits transmitted by the other end node by subtracting its own information sequence from the sequence detected from the symbol transmitted by the relay: $\hat{\mathbf{u}}_{2}=\hat{\mathbf{u}} \oplus \mathbf{u}_{1}$ at $\mathcal{N}_{1}$ and $\tilde{\mathbf{u}}_{1}=\tilde{\mathbf{u}} \oplus \mathbf{u}_{2}$ at $\mathcal{N}_{2}$. Since the links from the relay to the end nodes are conventional point-to-point links with no interfering transmissions, specific details of the end node receivers are omitted.

\subsection{Digital Network-Coded Relay Demodulator}

The DNC relay demodulator maps the received sum of symbols from the end nodes to log-likelihood ratios (LLRs) representing the network-coded bits. The demodulator operates iteratively, using information fed back from the channel decoder to refine LLRs during each decoding iteration. After a specified number of iterations has been reached, the decoder makes a hard decision on the network-coded bits.

The demodulator processes a frame of channel observations $\mathbf{Y}_{R}$ one observation at a time. Since the operation performed on each observation is the same, we may drop the dependence on a particular signaling interval in the frame to simplify the notation. Denote a single received channel observation as $\mathbf{y}_{R}$. During the first demodulation and decoding iteration, the demodulator computes the probability of receiving every possible combination 
of symbols transmitted by the end nodes: $P(q ; I)$, where $q$ is defined as the tuple

$$
q=\left(q_{1}, q_{2}\right) \quad q_{1}, q_{2} \in \mathcal{D} \quad q \in \mathcal{Q}
$$

where $q_{1}$ and $q_{2}$ denote the indices of the symbols from end node $\mathcal{N}_{1}$ and $\mathcal{N}_{2}$, respectively, and $\mathcal{Q}=\mathcal{D} \times \mathcal{D}$. Denote $q$ as a super-symbol. Define this operation as the super-symbol probability mapping stage. Since the cardinality of $\mathcal{Q}$ is $M^{2}$, the relay receiver computes $M^{2}$ probabilities, versus a conventional point-to-point receiver which only computes $M$ probabilities.

During each decoding iteration, the symbol probabilities are transformed to the set of $\mu$ LLRs associated with each network-coded bit mapped to the super-symbol. Denote this operation as digital network-coded soft mapping (DNC-SOMAP). A general description of SOMAP for the point-to-point channel is given by [66]. The DNC-SOMAP takes as input the symbol probabilities $P(q ; I)$ and a priori LLRs associated with each network-coded bit. The input LLR to the demodulator representing the $k$-th bit mapped to the super-symbol is denoted the a priori demodulator information and is related to the input distribution by

$$
v_{k}=\log \frac{P\left(b_{k}=1 ; I\right)}{P\left(b_{k}=0 ; I\right)}, 0 \leq k \leq \mu-1 .
$$

Considering BICM-ID, prior to the first decoding iteration, the bit probabilities are assumed equally likely, so the a priori LLRs are set to zero. The output LLR representing the $k$-th bit mapped to the super-symbol is denoted the a posteriori demodulator information and is related to the output distribution by

$$
z_{k}=\log \frac{P\left(b_{k}=1 ; O\right)}{P\left(b_{k}=0 ; O\right)}, 0 \leq k \leq \mu-1 .
$$

The DNC-SOMAP output distribution is related to the input distributions by

$$
P\left(b_{k}=\ell ; O\right)=\sum_{q: b_{k}(q)=\ell} p\left(\mathbf{y}_{R} \mid q\right) \prod_{\substack{j=0 \\ j \neq k}}^{\mu-1} P\left(b_{j}(q) ; I\right)
$$

where the function $b_{k}(q)$ selects the $k$-th network-coded bit associated with the super-symbol $q: b_{k}(q)=b_{k}\left(q_{1}\right) \oplus b_{k}\left(q_{2}\right)$. Substituting the specific values of the distribution Eq. (3.3) into 
the expression for output Eq. (3.5),

$$
P\left(b_{k}=\ell ; O\right)=\sum_{q: b_{k}(q)=\ell} p\left(\mathbf{y}_{R} \mid q\right) \prod_{\substack{j=0 \\ j \neq k}}^{\mu-1} \frac{e^{b_{j}(q) v_{j}}}{1+e^{v_{j}}} .
$$

The output LLR of the DNC-SOMAP may be found by combining Eq. (3.6) and Eq. (3.4):

$$
z_{k}=\log \left[\sum_{q: b_{k}(q)=1} p\left(\mathbf{y}_{R} \mid q\right) \prod_{\substack{j=0 \\ j \neq k}}^{\mu-1} e^{b_{j}(q) v_{j}}\right]-\log \left[\sum_{q: b_{k}(q)=0} p\left(\mathbf{y}_{R} \mid q\right) \prod_{\substack{j=0 \\ j \neq k}}^{\mu-1} e^{b_{j}(q) v_{j}}\right]
$$

where the term $\left(1+e^{v_{j}}\right)$ cancels in the ratio.

For numeric implementation, it is useful to simplify Eq. (3.7) using the max-star operator

$$
\max _{i} *\left\{x_{i}\right\}=\log \left\{\sum_{i} e^{x_{i}}\right\}
$$

where the binary max-star operator is $\max *(x, y)=\max (x, y)+\log \left(1+e^{-|x-y|}\right)$ and multiple arguments imply a recursive relationship; for example: $\max *(x, y, z)=\max *(x, \max *(y, z))$. Applying the max-star operator to Eq. (3.7)

$$
z_{k}=\max _{q: b_{k}(q)=1}\left[\log p\left(\mathbf{y}_{R} \mid q\right)+\sum_{\substack{j=0 \\ j \neq k}}^{\mu-1} b_{j}(q) v_{j}\right]-\max _{q: b_{k}(q)=0}\left[\log p\left(\mathbf{y}_{R} \mid q\right)+\sum_{\substack{j=0 \\ j \neq k}}^{\mu-1} b_{j}(q) v_{j}\right] .
$$

The values taken by the pdf $p\left(\mathbf{y}_{R} \mid q\right)$ are dependent on the available channel state information. Description of these pdfs is given in the following subsection.

\subsubsection{Super-Symbol Probability Distributions}

In this section, the distribution of the super-symbols, conditioned on the channel model and available CSI, is derived for successively relaxed assumptions of channel state information.

\section{Coherent Reception}

When conditioned on the fading coefficients and transmitted signals, the output of the matched-filters is the sum of two $M$-dimensional complex Gaussian vectors, which is itself 
Gaussian. Let $\mathbf{m}_{q}$ denote the mean of the received Gaussian vector when the symbols $q_{1}$ and $q_{2}$ are transmitted by the two end nodes. There are $M^{2}$ such vectors, each having form

$$
\mathbf{m}_{q}=h_{1} \mathbf{x}_{1}+h_{2} \mathbf{x}_{2}
$$

where $q=\left(q_{1}, q_{2}\right)$ denotes the super-symbol mapped to each pair of symbols transmitted by the end nodes. In AWGN, $\left|h_{1}\right|=\left|h_{2}\right|=1$. The coherent receiver computes $p\left(\mathbf{y} \mid q, \mathbf{m}_{q}\right)$ for all values of $q$. Since the event $\mathbf{m}_{q}$ implies $q$, we may drop dependence on $q$ and use the notation $p\left(\mathbf{y} \mid \mathbf{m}_{q}\right)$ to be more concise. Applying the definition of the pdf of an M-dimensional complex-Gaussian vector, it is found that

$$
p\left(\mathbf{y}_{R} \mid \mathbf{m}_{q}\right)=\left(\frac{1}{\pi N_{0}}\right)^{M} \exp \left\{-\frac{1}{N_{0}}\left\|\mathbf{y}_{R}-\mathbf{m}_{q}\right\|^{2}\right\} .
$$

\section{Noncoherent Reception with CSI}

In a Rayleigh fading channel, when the amplitudes of the fading coefficients are available at the receiver but the phases are not, the conditional pdf is found by marginalizing over the unknown phases of the received tones. In an AWGN channel the fading amplitudes are known and equal to unity.

When the end nodes transmit different symbols $\left(q_{1} \neq q_{2}\right)$, there will be two tones received, and therefore two phases to marginalize

$$
p\left(\mathbf{y}_{R} \mid \mu_{q}\right)=\int_{0}^{2 \pi} \int_{0}^{2 \pi} p\left(\theta_{1}\right) p\left(\theta_{2}\right) p\left(\mathbf{y}_{R} \mid \mathbf{m}_{q}\right) d \theta_{1} d \theta_{2}
$$

where $\mu_{q}=\left|\mathbf{m}_{q}\right|$ is a vector whose elements are the magnitudes of the corresponding elements of $\mathbf{m}_{q}$ and it is assumed that the two received phases are independent. By substituting Eq. (3.11) into (3.12) and assuming uniformly distributed phases, the conditional pdf is

$$
\begin{aligned}
p\left(\mathbf{y}_{R} \mid \mu_{q}\right)= & \left(\frac{1}{\pi N_{0}}\right)^{M} \prod_{\substack{k=1 \\
k \neq\left\{q_{1}, q_{2}\right\}}}^{M} \exp \left\{-\frac{\left|y_{k}\right|^{2}}{N_{0}}\right\} \times \ldots \\
& {\left[\frac{1}{2 \pi} \int_{0}^{2 \pi} \exp \left\{-\frac{\left|y_{q_{1}}-\alpha_{1} e^{j \theta_{1}}\right|^{2}}{N_{0}}\right\} d \theta_{1}\right]\left[\frac{1}{2 \pi} \int_{0}^{2 \pi} \exp \left\{-\frac{\left|y_{q_{2}}-\alpha_{2} e^{j \theta_{2}}\right|^{2}}{N_{0}}\right\} d \theta_{2}\right] } \\
= & \beta \exp \left\{-\frac{\alpha_{1}^{2}+\alpha_{2}^{2}}{N_{0}}\right\} I_{0}\left(\frac{2\left|y_{q_{1}}\right| \alpha_{1}}{N_{0}}\right) I_{0}\left(\frac{2\left|y_{q_{2}}\right| \alpha_{2}}{N_{0}}\right)
\end{aligned}
$$


where the factor

$$
\beta=\left(\frac{1}{\pi N_{0}}\right)^{M} \prod_{k=1}^{M} \exp \left\{-\frac{\left|y_{k}\right|^{2}}{N_{0}}\right\}
$$

is common to all possible pairs of symbols and cancels in the LLR. In AWGN, $\alpha_{1}=\alpha_{2}=1$.

When the end nodes transmit the same symbols $\left(q_{1}=q_{2}\right)$, the vector $\mathbf{m}_{q}$ will have a single nonzero entry $h=h_{1}+h_{2}=\alpha e^{j \phi}$ with phase $\phi=\angle\left(\alpha_{1} e^{j \theta_{1}}+\alpha_{2} e^{j \theta_{2}}\right)$ and amplitude

$$
\alpha=\left|\alpha_{1} \exp \left(j \theta_{1}\right)+\alpha_{2} \exp \left(j \theta_{2}\right)\right|
$$

The conditional pdf is found by marginalizing over $\phi$,

$$
p\left(\mathbf{y}_{R} \mid \mu_{q}\right)=\int_{0}^{2 \pi} p(\phi) p\left(\mathbf{y}_{R} \mid \mathbf{m}_{q}\right) d \phi .
$$

The tone is received through an equivalent fading channel with complex fading coefficient $h$. In Rayleigh fading $h_{1}$ and $h_{2}$ are zero-mean Gaussian, and it follows that $h$ is also zero-mean Gaussian and $\phi$ is uniform. Substituting the uniform pdf of $\phi$, the conditional pdf becomes

$$
\begin{aligned}
p\left(\mathbf{y}_{R} \mid \mu_{q}\right) & =\left(\frac{1}{\pi N_{0}}\right)^{M} \prod_{\substack{k=1 \\
k \neq q_{1}}}^{M} \exp \left\{-\frac{\left|y_{k}\right|^{2}}{N_{0}}\right\}\left[\frac{1}{2 \pi} \int_{0}^{2 \pi} \exp \left\{-\frac{\left|y_{i}-\alpha e^{j \phi}\right|^{2}}{N_{0}}\right\} d \phi\right] \\
& =\beta \exp \left\{-\frac{\alpha^{2}}{N_{0}}\right\} I_{0}\left(\frac{2\left|y_{q_{1}}\right| \alpha}{N_{0}}\right)
\end{aligned}
$$

where $\beta$ is given by Eq. (3.14). When the fading amplitude $\alpha$ is not known at the relay, and the amplitudes $\alpha_{1}$ and $\alpha_{2}$ are known, the receiver may approximate it as $\alpha=\sqrt{\alpha_{1}^{2}+\alpha_{2}^{2}}$ [23].

\section{Noncoherent Reception without CSI}

When the relay only has knowledge of the average received energy, the conditional pdf of the received signal is marginalized over the fading amplitudes. When the end nodes transmit different symbols, there are two fading amplitudes to marginalize over, and the conditional pdf becomes

$$
p\left(\mathbf{y}_{R} \mid q\right)=\int_{0}^{2 \pi} \int_{0}^{2 \pi} p\left(\alpha_{1}\right) p\left(\alpha_{2}\right) p\left(\mathbf{y}_{R} \mid \mu_{q}\right) d \alpha_{1} d \alpha_{2}
$$


where it is assumed that the $\alpha_{i}$ are independent, each with pdf

$$
p\left(\alpha_{i}\right)=\frac{2 \alpha_{i}}{\mathcal{E}_{i}} \exp \left\{-\frac{\alpha_{i}^{2}}{\mathcal{E}_{i}}\right\} .
$$

Substituting Eqs. (3.13) and (3.19) into (3.18) and then evaluating yields

$$
p\left(\mathbf{y}_{R} \mid q\right)=\beta\left[\left(\frac{1}{\mathcal{E}_{1} \mathcal{E}_{2}}\right)\left(\frac{1}{\mathcal{E}_{1}}+\frac{1}{N_{0}}\right)\left(\frac{1}{\mathcal{E}_{2}}+\frac{1}{N_{0}}\right)\right]^{-1} \exp \left\{\frac{\left|y_{q_{1}}\right|^{2} \mathcal{E}_{1}}{N_{0}\left(N_{0}+\mathcal{E}_{1}\right)}+\frac{\left|y_{q_{2}}\right|^{2} \mathcal{E}_{2}}{N_{0}\left(N_{0}+\mathcal{E}_{2}\right)}\right\}
$$

When the same tone is transmitted by both nodes, the marginalization is over the composite fading amplitude $\alpha$, and

$$
p\left(\mathbf{y}_{R} \mid q\right)=\int_{0}^{2 \pi} p(\alpha) p\left(\mathbf{y}_{R} \mid \mu_{q}\right) d \alpha .
$$

Recall that the tone is received over a fading channel with an equivalent complex-fading coefficient $h=h_{1}+h_{2}=\alpha e^{j \phi}$. The amplitude $\alpha$ is Rayleigh with pdf

$$
p(\alpha)=\frac{2 \alpha}{\mathcal{E}_{1}+\mathcal{E}_{2}} \exp \left\{-\frac{\alpha^{2}}{\mathcal{E}_{1}+\mathcal{E}_{2}}\right\} .
$$

Substituting Eqs. (3.17) and (3.22) into (3.21) and evaluating yields

$$
p\left(\mathbf{y}_{R} \mid q\right)=\beta\left(\frac{1}{\mathcal{E}_{1}+\mathcal{E}_{2}}\right)\left(\frac{1}{\mathcal{E}_{1}+\mathcal{E}_{2}}+\frac{1}{N_{0}}\right)^{-1} \exp \left\{\frac{\left|y_{q_{1}}\right|^{2}\left(\mathcal{E}_{1}+\mathcal{E}_{2}\right)}{N_{0}^{2}+N_{0}\left(\mathcal{E}_{1}+\mathcal{E}_{2}\right)}\right\} .
$$

\subsection{Capacity}

In this section, the capacity of LNC and DNC is analyzed and simulated. Specifically, expressions for CM capacity [34] suitable for Monte Carlo simulation are derived and used to generate capacity curves. CM capacity provides a limit on energy efficiency when feedback is present between the channel decoder and demodulator, an arrangement that approximates joint demodulation and decoding [67].

\subsubsection{End-to-End Capacity Analysis}

All node communication is assumed half-duplex, thus, information exchange between the end nodes occurs in two sequential time phases. In the MA phase, the end nodes transmit 
information to the relay, and the relay detects the network-coded combination of information bits from the end nodes. In the $\mathrm{BC}$ phase, the relay broadcasts the network-coded bits to the end nodes. Each end node detects the bits transmitted by the opposite end node by performing channel decoding on the network-coded bits and subtracting its own bits.

During the MA phase, the goal of the relay is to detect the network-coded combination of bits $\mathbf{b}=\mathbf{b}_{1} \oplus \mathbf{b}_{2}$ on a symbol-by-symbol basis. This channel may be modeled as a virtual single-input single-output channel having input $\mathbf{b}$ and channel output $\mathbf{y}_{R}$, thus, the transition distribution during the MA phase is $p\left(\mathbf{y}_{R} \mid \mathbf{b}\right)$ [68]. Assuming uniformly distributed inputs $\mathbf{b}_{1}$ and $\mathbf{b}_{2}$ at the end nodes, the capacity during the MA phase is given by the conditional average mutual information (AMI) $I\left(\mathbf{b} ; \mathbf{y}_{R}\right)[34]$.

During the BC phase, the relay broadcasts the network-coded information bits to the end nodes. In this work it is assumed that that the statistics of the channels between the relay and each end node are identical. Under this assumption we may model the broadcast phase as a conventional point-to-point channel having transition probability $p\left(\mathbf{y}_{e} \mid \mathbf{b}\right)$ and capacity $I\left(\mathbf{b} ; \mathbf{y}_{e}\right)$, where $\mathbf{y}_{e}$ is the received signal at the end nodes. Since the capacity of FSK in the point-to-point channel is well known, we omit derivation in this work. A thorough treatment may be found in [58].

The end-to-end capacity is a function of the MA and $\mathrm{BC}$ capacities and the fraction of time allocated to each phase. Each phase may be modeled as a cascade of point-to-point links, thus, the end-to-end capacity is given by the max-flow min-cut theorem [69]

$$
C_{E}=\max _{t_{m}} \min \left\{t_{m} I\left(\mathbf{b} ; \mathbf{y}_{R}\right),\left(1-t_{m}\right) I\left(\mathbf{b} ; \mathbf{y}_{e}\right)\right\}
$$

where $t_{m}$ is the fraction of time assigned to the MA phase and $1-t_{m}$ is the fraction of time assigned to the BC phase. The end-to-end capacity is maximized by allocating transmission time to each phase such that time-scaled capacities are equalized

$$
\begin{aligned}
t_{m} I\left(\mathbf{b} ; \mathbf{y}_{R}\right) & =\left(1-t_{m}\right) I\left(\mathbf{b} ; \mathbf{y}_{e}\right) . \\
t_{m} & =\frac{I\left(\mathbf{b} ; \mathbf{y}_{e}\right)}{I\left(\mathbf{b} ; \mathbf{y}_{R}\right)+I\left(\mathbf{b} ; \mathbf{y}_{e}\right)}
\end{aligned}
$$


Substituting Eq. (3.25) into (3.24) yields

$$
C_{E}=\frac{I\left(\mathbf{b} ; \mathbf{y}_{R}\right) I\left(\mathbf{b} ; \mathbf{y}_{e}\right)}{I\left(\mathbf{b} ; \mathbf{y}_{R}\right)+I\left(\mathbf{b} ; \mathbf{y}_{e}\right)} .
$$

In the following subsection, capacity is derived for the MA phase.

\subsubsection{Capacity Analysis for Multiple-Access Phase}

In this subsection, capacity during the multiple-access phase is computed for DNC and LNC. Expressions for CM capacity are derived. Capacities are normalized by the bitsper-symbol, expressing capacity in units of channel symbols per channel use, which can be interpreted as the maximum error-free binary channel code rate. Expressing capacity in this manner enables performance comparison between different modulation orders.

Considering DNC, the end nodes transmit simultaneously to the relay during the MA phase, yielding the following received signal for a single symbol period

$$
\mathbf{y}_{R}=h_{1, R} \mathbf{x}_{1}+h_{2, R} \mathbf{x}_{2}+\mathbf{n}_{R}
$$

where $h_{1, R}$ and $h_{2, R}$ are complex channel gains and $\mathbf{n}_{R}$ is additive white Gaussian noise. The transition distribution for this channel is $p\left(\mathbf{y}_{R} \mid \mathbf{x}_{1}, \mathbf{x}_{2}\right)$. At source $\mathcal{N}_{i}$, denote the information bit sequence $\mathbf{b}_{i}$ mapped to channel symbol index $q_{i}$ as $\mathbf{b}_{i}\left(q_{i}\right)$. Denote the decimal representation of a bit sequence as $d\left(\mathbf{b}_{i}\right)$, for instance, $d(10)=2$. The network-coded symbol indices are defined as the random variable $q$ taking values from $\mathcal{Q}$ and mapped to the end node symbol indices as

$$
q=d\left(\mathbf{b}_{1}\left(q_{1}\right) \oplus \mathbf{b}_{2}\left(q_{2}\right)\right) .
$$

The CM capacity of the DNC multiple-access phase is then defined as

$$
C_{D, M}=I\left(q ; \mathbf{y}_{R}\right)=1-\frac{1}{\mu} E_{q, \mathbf{y}_{R}}\left[\log _{2} \frac{\sum_{\left(\mathbf{x}_{1}, \mathbf{x}_{2}\right) \in \mathcal{X} \times \mathcal{X}} p\left(\mathbf{y}_{R} \mid \mathbf{x}_{1}, \mathbf{x}_{2}\right)}{\sum_{\left(\mathbf{x}_{1}, \mathbf{x}_{2}\right) \in \mathcal{X} \times\left.\mathcal{X}\right|_{q}} p\left(\mathbf{y}_{R} \mid \mathbf{x}_{1}, \mathbf{x}_{2}\right)}\right]
$$

where $\mathcal{X} \times\left.\mathcal{X}\right|_{q}$ denotes all combinations of channel symbols $\mathbf{x}_{1}$ and $\mathbf{x}_{2}$ such that $d\left(\mathbf{b}_{1}\left(q_{1}\right) \oplus\right.$ $\left.\mathbf{b}_{2}\left(q_{2}\right)\right)=q$. 
In the LNC MA phase, the end nodes transmit in separate time slots to the relay, yielding the pair of received symbols

$$
\mathbf{y}_{1, R}=h_{1, R} \mathbf{x}_{1}+\mathbf{n}_{1, R} \quad \mathbf{y}_{2, R}=h_{2, R} \mathbf{x}_{2}+\mathbf{n}_{2, R}
$$

which may be modeled as two separate point-to-point channels during each time slot having transition distribution $p\left(\mathbf{y}_{k, R} \mid \mathbf{x}_{k}\right), k \in\{1,2\}$. In this work we assume that each end node is assigned one-half of the MA phase transmission time: $t_{m} / 2$. Thus, the MA capacity for LNC may be modeled as a time-division multiple-access (TDMA) system where capacity is one-half the capacity of a conventional point-to-point system [2]. The CM capacity for LNC is defined as

$$
C_{L, M}=\frac{1}{2} I\left(q_{k} ; \mathbf{y}_{k, R}\right)=\frac{1}{2}-\frac{1}{2 \mu} E_{q_{k}, \mathbf{y}_{k, R}}\left[\log _{2} \frac{\sum_{\mathbf{x}_{k} \in \mathcal{X}} p\left(\mathbf{y}_{k, R} \mid \mathbf{x}_{k}\right)}{p\left(\mathbf{y}_{k, R} \mid \mathbf{x}_{k}\left(q_{k}\right)\right)}\right]
$$

where $\mathbf{x}_{k}\left(q_{k}\right)$ denotes the channel symbol $\mathbf{x}_{k}$ mapped to symbol index $q_{k}$, and the factor $\frac{1}{2}$ accounts for the TDMA characteristic of LNC. We assume identical signaling schemes and channels between each end node and the relay, thus $I\left(\mathbf{b}_{1} ; \mathbf{y}_{1, R}\right)=I\left(\mathbf{b}_{2} ; \mathbf{y}_{2, R}\right)$ and $I\left(q_{1} ; \mathbf{y}_{1, R}\right)=$ $I\left(q_{2} ; \mathbf{y}_{2, R}\right)$.

\subsubsection{Simulated Capacity}

This section presents simulated capacity for DNC and LNC. Multiple-access phase and end-to-end capacity is shown. FSK modulation orders two, four and eight are simulated. AWGN and Rayleigh fading channels are considered. In fading, demodulation during the MA phase is simulated with and without CSI at the relay. The difference in performance between DNC and LNC is discussed.

Computing MA phase capacity by the Monte Carlo method begins by simulating frame transmission to the relay. A range of SNR values is specified, expressed as the ratio of symbol energy to noise power $\mathcal{E}_{S} / N_{0}$, and $\mathcal{E}_{S}=\mathcal{E}_{1}=\mathcal{E}_{2}$. SNR is expressed in terms of energy per bit as $\mathcal{E}_{b} / N_{0}=\frac{\mathcal{E}_{S} / N_{0}}{C \log _{2} M}$, where $C$ denotes the simulated capacity. An FSK modulation order $M$ 


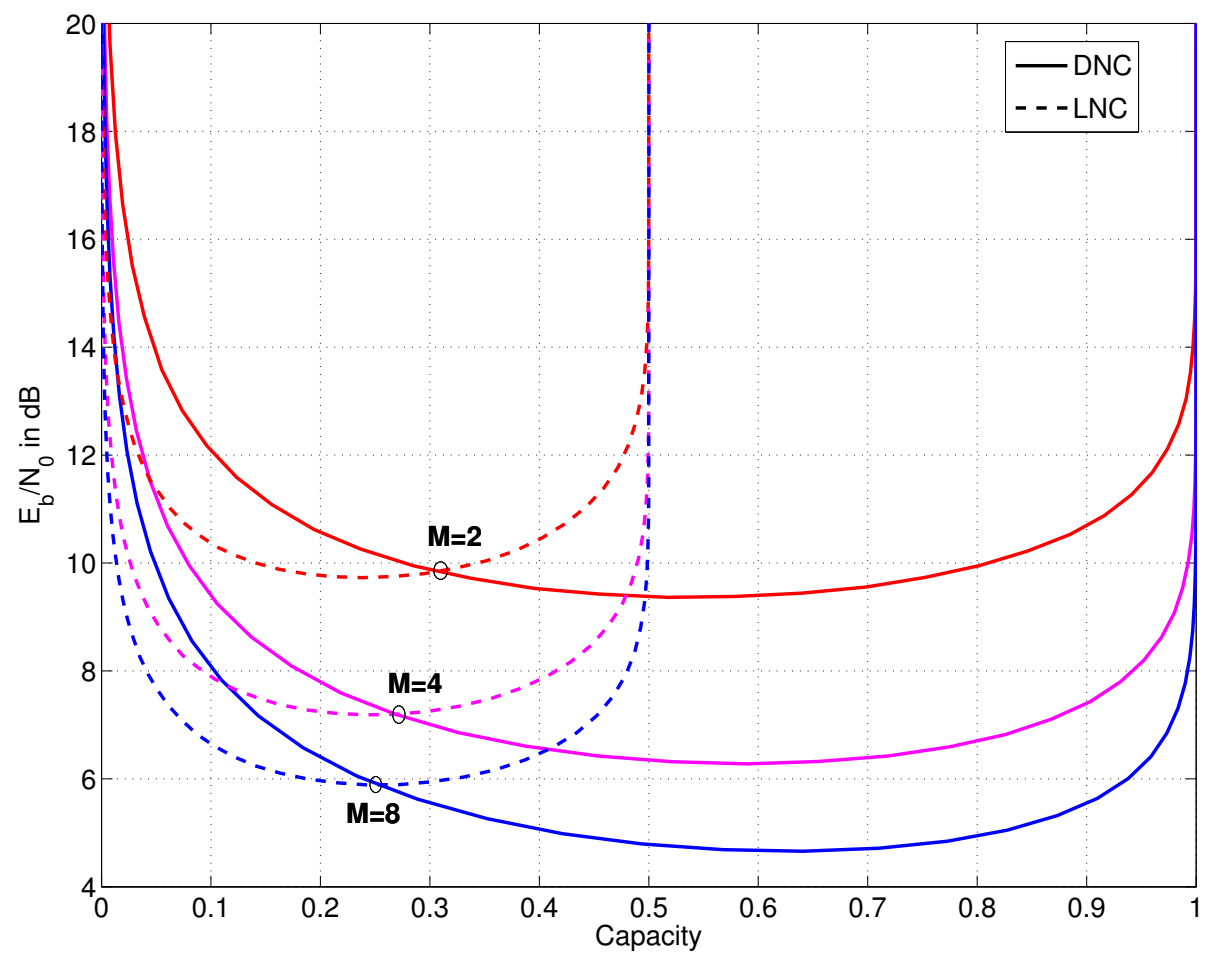

Figure 3.3: Capacity for the TWRC MA phase in AWGN with random phase noise. Solid and dashed lines denote DNC and LNC respectively. Modulation orders are $M=\{2,4,8\}$.

is selected. Each end node generates a binary information sequence and maps it to $M$-ary FSK symbols as described in Section 1.3.1. The channel effects on the symbols transmitted from the end nodes to the relay are simulated according to Eq. (1.3) for DNC and Eq. (1.4) for LNC. In fading, the amplitudes of the fading coefficients are Rayleigh distributed according to Eq. (1.2) with $\mathcal{E}_{i}=1$, while in AWGN the fading amplitudes are set to unity.

MA phase capacity is computed using the received symbol frame and network-coded bit values. Capacity for DNC is computed by substituting Eq. (3.8) into (3.29)

$$
C_{D, M}=1-\frac{\log _{2}(e)}{\mu} E_{q, \mathbf{y}_{R}}\left[\max _{\left(\mathbf{x}_{1}, \mathbf{x}_{2}\right) \in \mathcal{X} \times \mathcal{X}} \log p\left(\mathbf{y}_{R} \mid \mathbf{x}_{1}, \mathbf{x}_{2}\right)-\underset{\left(\mathbf{x}_{1}, \mathbf{x}_{2}\right) \in \mathcal{X} \times\left.\mathcal{X}\right|_{q}}{\max *} \log p\left(\mathbf{y}_{R} \mid \mathbf{x}_{1}, \mathbf{x}_{2}\right)\right]
$$

and for LNC by substituting Eq. (3.8) in (3.31)

$$
C_{L, M}=\frac{1}{2}-\frac{\log _{2}(e)}{2 \mu} E_{q_{k}, \mathbf{y}_{k, R}}\left[\max _{\mathbf{x}_{k} \in \mathcal{X}} \log p\left(\mathbf{y}_{k, R} \mid \mathbf{x}_{k}\right)-\max _{\mathbf{x}_{k} \in \mathcal{X}} * \log p\left(\mathbf{y}_{k, R} \mid \mathbf{x}_{k}\left(q_{k}\right)\right)\right] .
$$

The expectations are evaluated for several hundred thousand Monte Carlo trials. 


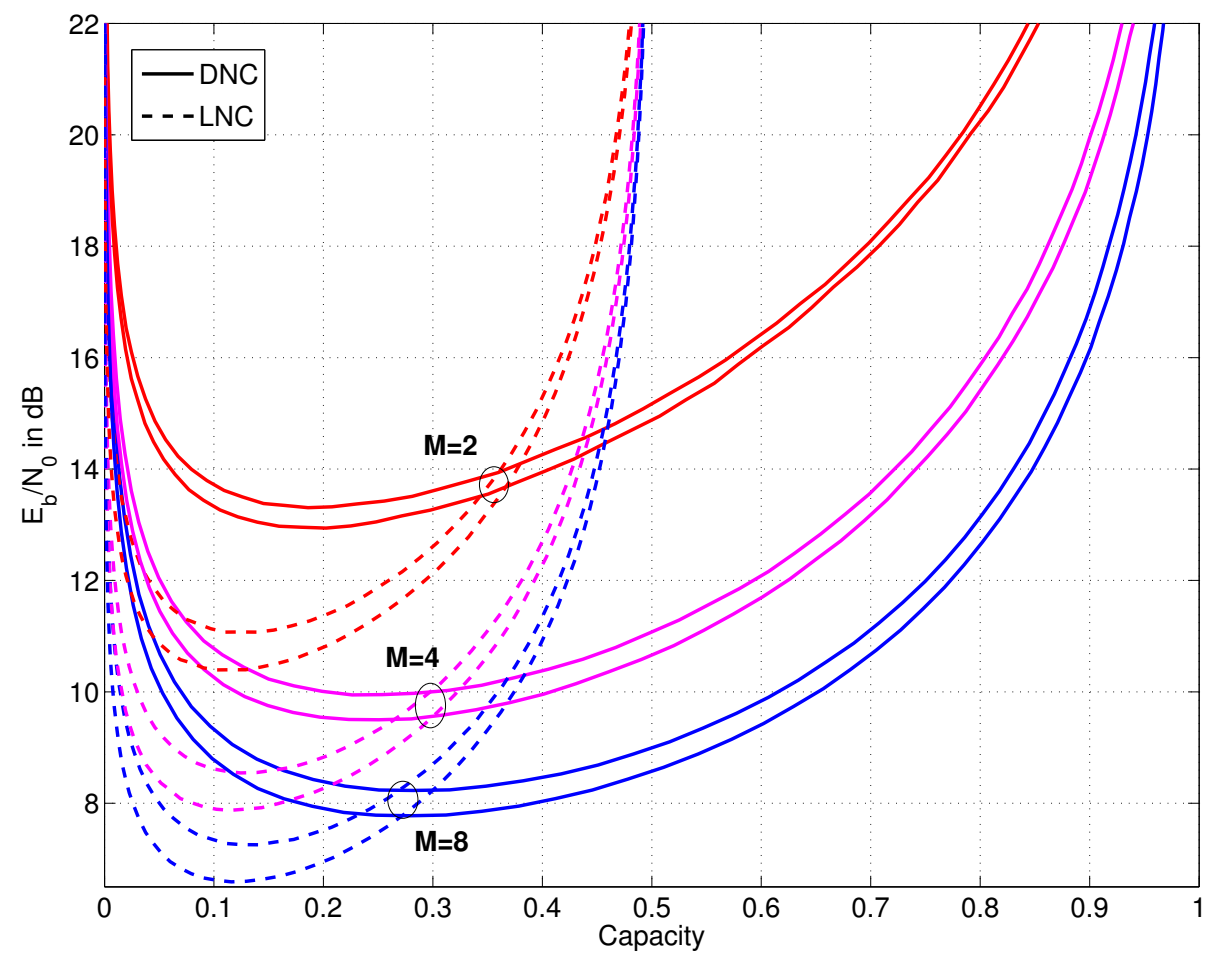

Figure 3.4: Capacity for the TWRC MA phase in Rayleigh fading. Modulation orders are $M=\{2,4,8\}$. For DNC and LNC at every modulation order, a pair of similar curves is shown. Within each pair, the upper and lower curves depict capacity for partial and no CSI at the relay, respectively.

Capacity in AWGN is shown in Fig. 3.3. DNC capacity takes values between 0 and 1 while LNC capacity takes values between 0 and 0.5 , demonstrating that DNC enables higher rates than possible for LNC. At rates less than 0.5, distinct regions exist where either DNC or LNC is more energy efficient. At modulation orders two, four and eight, LNC is more energy efficient at rates less than approximately $0.3,0.27$ and 0.25 respectively, while DNC is more efficient at higher rates. In general, the range of rates where DNC energy efficiency outperforms LNC increases with modulation order.

Capacity in Rayleigh fading with and without CSI at the relay is shown in Fig. 3.4. The maximum performance improvement of CSI over no CSI is approximately 1 and $0.5 \mathrm{~dB}$ for LNC and DNC respectively, indicating that CSI is more beneficial for LNC. Consider performance at rates less than 0.5. LNC exhibits better energy efficiency than DNC at 


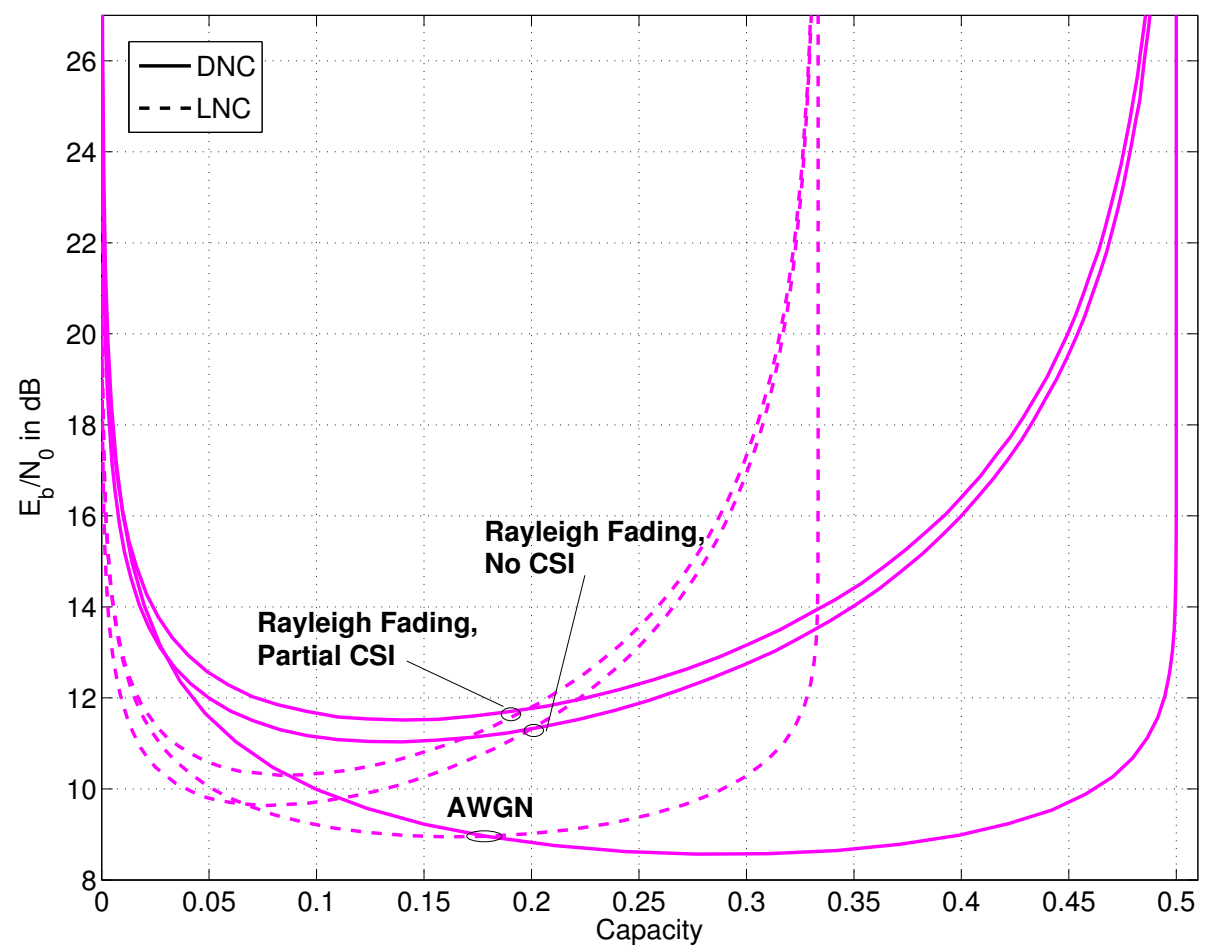

Figure 3.5: End-to-end capacity in AWGN and Rayleigh fading with no CSI and partial CSI for digital and link-layer network coding (DNC and LNC). Modulation order $M=4$ is shown.

approximate rates $0.35,0.3$, and 0.26 when the relay has no CSI and rates $0.36,0.3$ and 0.27 when the relay has partial CSI, at modulation orders two, four and eight, respectively. LNC outperforms DNC over a wider range of rates in fading than in AWGN, demonstrating that fading degrades the capacity of DNC more severely than LNC.

End-to-end capacity is shown in Fig. 3.5. Capacity is shown assuming partial and no $\mathrm{CSI}$ at the relay and for modulation order $M=4$. The maximum capacities for DNC and LNC are $1 / 2$ and $1 / 3$ respectively, which is consistent with DNC requiring two time slots to exchange information and LNC requiring three. In AWGN, the energy efficiency of DNC outperforms LNC at rates greater than approximately 0.17. In fading, DNC outperforms LNC at rates greater than 0.18 and 0.2. The maximum energy efficiency difference between partial and no CSI is about $0.5 \mathrm{~dB}$ for LNC and about $0.25 \mathrm{~dB}$ for DNC. 


\subsection{LDPC Coded Performance}

This section presents LDPC-coded error rate performance and optimization for the TWRC multiple-access stage when combined with the DNC relay demodulator described in Section 3.3. Error rates are computed via Monte Carlo simulation. Performance is investigated using the LDPC code defined by the DVB-S2 standard. The LNC protocol is simulated and compared to DNC.

\subsubsection{Bit Error Rate Simulation Procedure}

Throughout this section, the following procedure is applied to simulate LDPC-coded bit-error rate (BER) performance during the TWRC MA stage. A range of SNR values is specified, expressed as the ratio of bit energy to noise power $\mathcal{E}_{b} / N_{0}$. An FSK modulation order $M$ is selected. Each end node generates a binary information sequence, performs LDPC encoding using the appropriate parity check matrix to produce a codeword, and maps the codeword to $M$-ary FSK symbols as described in Section 1.3.1. The channel effects on the symbols transmitted from the end nodes to the relay are simulated according to Eq. (1.3) for DNC and Eq. (1.4) for LNC. In fading, the amplitudes of the fading coefficients are Rayleigh distributed according to Eq. (1.2) with $\mathcal{E}_{i}=1$, while in AWGN the fading amplitudes are set to unity.

BER performance for standard codes is computed using parity check matrices defined by the DVB-S2 standard [70]. In order to fairly compare performance between DNC and LNC during the MA stage, the number of information bits sent to the relay by each end node and the total number of symbol periods is assumed the same for both protocols. The MA rates considered are $r_{M}=\{1 / 3,2 / 5,3 / 5\}$, thus, $r_{D}=\{1 / 3,2 / 5,3 / 5\}$ and $r_{L}=\{2 / 3,4 / 5\}$. The channel code lengths for DNC and LNC are $N_{D}=16200$ and $N_{L}=8100$ respectively.

The DVB-S2 standard does not specify codes having the length $N_{L}=8100$ as considered

for LNC, thus, parity check matrices are generated having degree distributions identical to DVB-S2 codes with length and rates $16200,2 / 3$, and $4 / 5$ respectively. The procedure for 


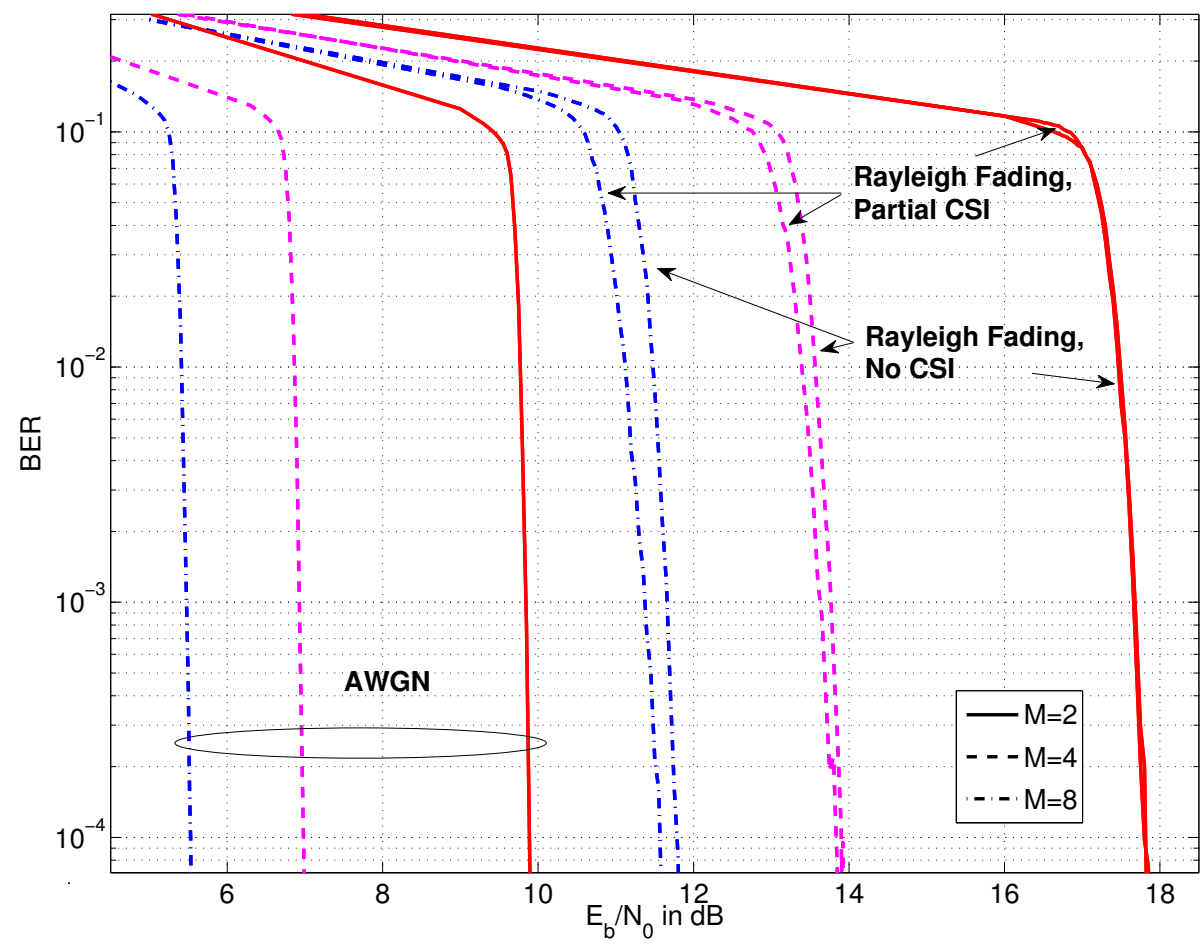

Figure 3.6: LDPC-coded BER performance at the relay for digital network coding in AWGN and Rayleigh fading channels using a DVB-S2 LDPC code. The code length and rate are $N_{D}=16200$ bits and $r_{D}=3 / 5$ respectively. FSK modulation orders $M=\{2,4,8\}$ are simulated. In fading, performance with partial and no channel state information at the relay is shown.

generating random parity check matrices is given in subsection 4.2.1.

The relay performs demodulation and decoding to detect the network-coded bits that are used to compute the BER. Considering DNC the relay demodulates the received symbols using Eq. (3.9) with $p\left(\mathbf{y}_{R} \mid q\right)$ corresponding to the desired relay CSI as given in subsection 3.3.1. BICM-ID decoding is performed as described in subsection 3.2.1. The number of decoding iterations is 100 for all simulations, as further iterations do not significantly improve decoding performance. Considering LNC, demodulation for each subframe is performed by conventional point-to-point techniques as described in [58]. Several hundred thousand simulation trials are performed, and the number of network-coded bits in error is counted and used to compute the BER. 


\subsubsection{Channel-Coded Performance}

To investigate the performance of the relay demodulator developed in Section 3.3 when coupled with soft-output channel coding, this subsection presents the results of LDPC-coded error rate simulation. The channel code is defined by the DVB-S2 standard [70]. Error rate performance in AWGN and Rayleigh fading is simulated. In fading, decoding is performed with and and without channel state information at the relay, as described in subsections 3.3.1 and 3.3.1. LNC is simulated for comparison.

Error-rate performance of DNC using the DVB-S2 LDPC code having rate $r_{D}=3 / 5$ is shown in Fig. 3.6. This figure illustrates performance considering all channels and CSI cases and three modulation orders. At modulation order $M=2$, the performance in fading is nearly identical regardless of the CSI available at the relay. The difference in performance between partial and no CSI is about 0.1 and $0.2 \mathrm{~dB}$ at $M=4$ and $M=8$, respectively. In fading, increasing modulation order from 2 to 4 and 4 to 8 improves energy efficiency by approximately 4 and $2 \mathrm{~dB}$, respectively. Similar behavior is observed in AWGN but with smaller performance differences between modulation orders. A 3 dB improvement when increasing modulation order from 2 to 4 and about $1 \mathrm{~dB}$ of improvement when increasing from modulation order 4 to 8 . At an error rate of $10^{-4}$, in AWGN, the difference between BER performance and capacity is about $0.5,0.6$ and $0.7 \mathrm{~dB}$ for modulation orders $M=\{2,4,8\}$ respectively. In fading, the difference is about 1.5, 2 and $2 \mathrm{~dB}$ respectively.

The bit-error rate performance of DNC and LNC at two different code rates is shown in Fig. 3.7. All simulations consider Rayleigh fading with partial CSI at the relay demodulator. At modulation order $M=2$ and MA rate $r_{M}=2 / 5$, DNC outperforms LNC by approximately $1.5 \mathrm{~dB}$, however at $r_{M}=1 / 3 \mathrm{LNC}$ outperforms DNC by about $0.5 \mathrm{~dB}$. Considering modulation order $M=4$, DNC outperforms LNC by approximately $4 \mathrm{~dB}$ at $r_{M}=2 / 5$ and by roughly $0.5 \mathrm{~dB}$ at $r_{M}=1 / 3$. Generally, increasing modulation order increases the performance difference between DNC and LNC favorably for DNC. At rate $r_{M}=2 / 5$, the difference between BER performance at $10^{-4}$ and capacity is about $1.1 \mathrm{~dB}$ for DNC and 1.5 $\mathrm{dB}$ for $\mathrm{LNC}$, on average. At rate $r_{M}=1 / 3$, the difference is about $1 \mathrm{~dB}$ and $0.7 \mathrm{~dB}$ for $\mathrm{DNC}$ 


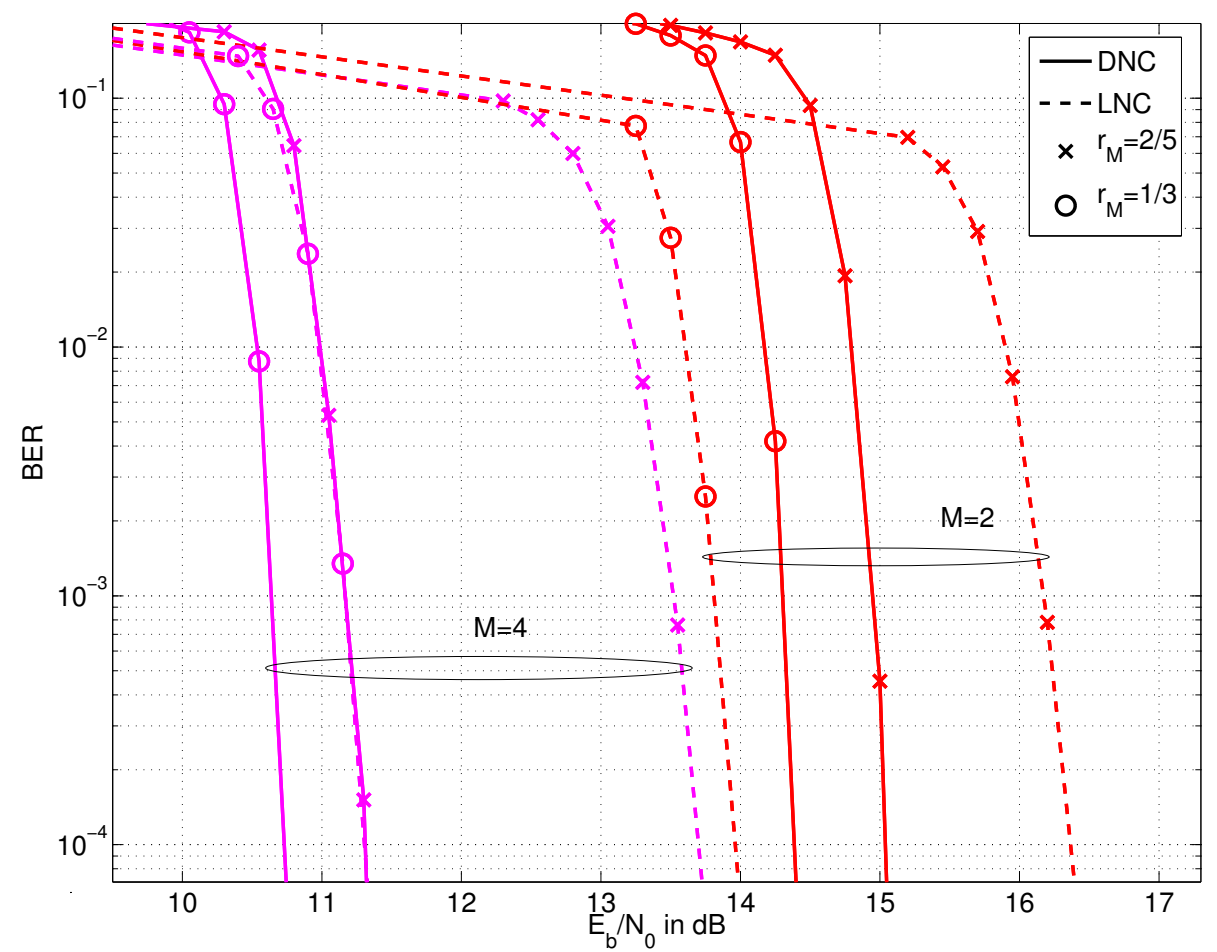

Figure 3.7: LDPC-coded BER performance at the relay for digital and link-layer network coding (DNC and LNC) in Rayleigh fading at channel code rates $r_{M}=\{2 / 5,1 / 3\}$. The relay possesses partial CSI as fading amplitudes. The DNC and LNC frame lengths and rates are $N_{D}=16200$ and $N_{L}=8100$ bits. FSK modulation orders $M=\{2,4\}$ are considered.

and $\mathrm{LNC}$ respectively.

\subsection{Conclusion}

This chapter has developed a soft-output M-FSK relay demodulator performing physicallayer network coding in the two-way relay channel. The demodulator supports noncoherent operation, power-of-two modulation orders and incorporates channel state information in the form of fading amplitudes. Feedback from the decoder to the demodulator refines the symbol likelihoods in a BICM-ID structure, improving bit error rate performance. The CM capacity of the TWRC considering both physical-layer network coding and conventional networking was analyzed and simulated. Generally, the capacity and error rate performance of physical 
layer network coding outperforms conventional network coding at high code rates, where conventional network coding is superior at low rates. A simulation campaign investigated the performance of the demodulator, specifically, modulation orders $M=\{2,4,8\}$ were simulated with and without fading amplitude knowledge and using LDPC channel coding. 


\section{Chapter 4}

\section{LDPC Code Design for DNC}

The previous chapters have developed a soft-output demodulation scheme for M-ary FSK in multiple-access stage of the two-way relay channel. In this chapter, we focus on optimizing the decoding performance and thus the energy efficiency of LDPC channel coding when coupled with the developed demodulator. An optimization procedure using extrinsic information transfer is applied to design LDPC code degree distributions. The performance of the optimized codes are compared against standardized codes and channel capacity.

\subsection{Introduction}

It has been shown that optimizing channel codes for particular channels and modulation strategies yields performance benefits [71]. Several works have considered optimizing codes for the TWRC. An irregular repeat-accumulate code optimized for the relay in AWGN using extrinsic information transfer (EXIT) charts [44] exhibits a considerable performance improvement over regular repeat-accumulate codes [72]. Optimizing low density parity check (LDPC) degree distributions using density evolution improves performance over codes designed for point-to-point channels [73]. LDPC degree distributions optimized using EXIT charts improve performance in the PNC multiple-access stage [40]. In this work, we optimize a channel coding scheme for the MA stage by using EXIT charts to design LDPC degree distributions suitable for the TWRC with fading. 
The error-rate performance of LDPC codes depends on the structure of the parity check matrix and thus the properties of the Tanner Graph and degree distribution. In this work we apply an optimization technique which identifies variable node degree distributions likely to yield good codes using EXIT curve fits [38]. LDPC codes taken from the DVB-S2 [70] and WiMAX [74] standard are used as a basis for design and performance comparison, and their variable node degree distributions are optimized to yield codes exhibiting error-rate performance superior to the standard codes. The complexity characteristics of the standard codes are maintained in the optimized codes by preserving the extended irregular repeataccumulate constraint. LDPC code optimization for the relay receiver was developed in [31], where it was shown that a combination of optimized codes and iterative decoding improves decoding performance significantly over standard channel codes decoded without feedback. A detailed discussion of encoding and decoding techniques for DVB-S2 including simulated performance is described in [75].

A variety of optimization techniques exist for improving LDPC code performance. Random permutation matrices, large girth optimization, and column weight optimization have been applied to WiMax standardized codes yielding gains up to $0.4 \mathrm{~dB}$ [39]. Joint design of LDPC codes under carrier frequency offsets demonstrate the relationship between system properties and code performance [76].

A PNC scheme compensating for correlation among transmitted symbols introduced by channel coding to improve decoder performance at the relay has been considered [77]. In this work it is demonstrated that LDPC codes having different degree distributions are preferred in different SNR regimes. Roughly, smaller degrees are preferable at low SNR and higher degrees at high SNR. A PNC system used as a basis for optimizing LDPC codes via EXIT analysis demonstrated performance $1.5 \mathrm{~dB}$ from capacity [40].

Degree distribution optimization for specific system configurations has been considered by other works. A joint source-channel coding system employing LDPC codes has considered the degree distribution optimization technique described in [38], observing performance improvements of $2-4 \mathrm{~dB}$ for the system considered [41]. In [78], a the degree distribution 
of a distributed LDPC code is optimized for the LNC two-way relay channel.

The rest of this work is organized as follows. Section 4.2 describes the variable node degree distribution optimization technique in information-theoretic terms, while Section 4.3 describes the computational procedure followed to generate the optimized codes and demonstrates the performance of the codes versus standard. Section 4.4 provides concluding remarks.

\subsection{LDPC Code Optimization}

This section describes optimization of LDPC code error-rate performance based on [38] under a range of receiver configurations in the DNC relay reception stage by varying the LDPC code degree distribution. Relevant LDPC concepts are introduced, followed by a description of metrics relevant to optimization. A theoretical description of the optimization technique is provided followed by the procedure taken.

Note that the system model used in this chapter is identical to Chapter 3. Specifically, LDPC codes are optimized for use with the system incorporating the DNC relay receiver developed in Chapter 4. Transmission by the end-nodes in the multiple access stage follows the model described in the introductory system model section, 1.3.1, and the assumed channel is described in the introductory channel model section, 1.3.2. The relay receiver used during the multiple-access stage is described in Section 3.3. The system model is the depicted graphically by the system model figure given in Chapter 3, Fig. 3.1.

The goal is to produce LDPC code designs having the same rate and complexity as off-the-shelf codes from the DVB-S2 and WiMAX standards, while improving error rate performance through optimization of the parity check matrix. The codes are constrained to the class of extended irregular repeat accumulate (eIRA) codes. This constraint ensures computational efficiency by guaranteeing systematic encoding [79]. The codes considered are check-regular, meaning that every check node has the same degree, $d_{c}$.

An LDPC code is a linear block code that may be fully described by a sparse binary 
parity check matrix $\mathbf{H}$. The dimensionality of $\mathbf{H}$ is $N-K$ rows by $N$ columns. Parity check matrices may be represented by a Tanner graph, a bipartite graph where the nodes are partitioned into two sets: variable nodes and check nodes. The graph contains $N$ variable nodes and $N-K$ check nodes, one for each column and row of $\mathbf{H}$ respectively. An edge connecting the $n$-th variable node to the $k$-th check node corresponds to a 1 in the parity check matrix located at row and column $(k, n)$. The degree of a variable or check node is the number of 1's in column $n$ of $\mathbf{H}$, and the degree of check node $k$ is the number of 1's in row $k$ of $\mathbf{H}$.

LDPC variable and check nodes may be modeled as a posteriori probability decoders which convert a priori input LLRs to extrinsic output LLRs [38]. The transfer characteristics of the variable and check node decoders may be characterized by measuring the mutual information between the a priori and extrinsic LLRs. Specifically, plotting the mutual information of the a priori LLRs against the extrinsic yields an EXIT curve. Matching the variable and check node EXIT curves as closely as possible through selection of variable node degree yields good LDPC decoding performance [80].

A soft-output demodulator which produces LLRs for received bits may be modeled jointly with the LDPC variable nodes to produce an EXIT curve characterizing the demodulator and the variable node decoders [38]. LDPC decoding performance may be optimized by matching the EXIT characteristic of the combined demodulator and variable node decoder with the check node decoder. In this section we develop optimized LDPC codes having EXIT characteristics matched to the DNC relay demodulator developed in Section 3.3. The performance of the optimized codes is compared to standard codes and the capacities calculated in Section 3.4.

An EXIT chart visualizes the transfer characteristic of a decoder by plotting the a-priori mutual information against the extrinsic. It has been shown that matching the VND and CND transfer characteristics of a particular LDPC code through selection of variable node degrees improves error rate performance [80]. 


\subsubsection{Optimization through Selection of Variable Node Degree}

The variable node degrees for an LDPC code are denoted by the set $\left\{d_{v, 1}, \ldots, d_{v, D}\right\}$, where $d_{v, i}$ is the $i$-th degree and $D$ is the number of distinct degrees. The degree distribution is defined as the set of variable node degrees and the number of nodes taking a particular degree $V=\left\{d_{v, 1}: o_{1}, \ldots, d_{v, D}: o_{D}, d_{c}\right\}$ where $o_{i}$ is the number of variable nodes having degree $d_{v, i}$.

A valid degree distribution satisfies the constraints imposed by the LDPC code parameters. The total number of edges incident on the variable and check nodes must be the same. The number of edges incident on variable nodes having degree $d_{v, i}$ is $e_{v, i}=o_{i} d_{v, i}$, thus, the total number of edges incident on all variable nodes is

$$
e_{v}=\sum_{i=1}^{D} o_{i} d_{v, i}
$$

and $\sum_{i=1}^{D} o_{i}=N$. In this work we consider LDPC codes having a single check node degree, described as check regular codes. The total number of edges incident on the check nodes is then $e_{c}=d_{c}(N-K)$. Equating $e_{v}$ and $e_{c}$ and rearranging,

$$
\sum_{i=1}^{D} \frac{o_{i} d_{v, i}}{d_{c}(N-K)}=1
$$

A degree distribution which can be realized as a parity check matrix is formed by choosing values for $N, K$ and $V$ that satisfy (4.2). The design challenge is to select degree distributions that optimize error rate performance for particular channels and relay receiver configurations.

The optimization procedure requires generating EXIT curves corresponding to the variablenode decoder (VND) and check-node decoder (CND). The VND curve is described by the variable node degree distribution $V$ and the mutual information computed between the DNCSOMAP output and the network coded bits. The CND curve is completely described by the degree of the check nodes $d_{c}$, and is fixed for all variable node degree distributions.

Generation of the VND curve begins by computing the mutual information between the soft-values at the output of the DNC-SOMAP and the network-coded bits, denoted as the detector characteristic $I_{E, D E T}\left(I_{A, D E T}, \frac{E_{b}}{N_{0}}\right) . I_{A, D E T}$ is the mutual information between 
the DNC-SOMAP a-priori input and the network-coded bits, and $\frac{E_{b}}{N_{0}}$ is the signal-to-noise ratio of the super-symbol. The detector characteristic $I_{E, D E T}$ is generated by Monte Carlo simulation under the assumption that the a-priori input $I_{A, D E T}$ is conditionally Gaussian. The Gaussian assumption can be verified empirically using histograms of several decoding runs [58].

The VND curve is found by computing a separate curve for each variable node degree and combining each to form the final curve describing the entire variable node detector. The curve corresponding to the $i$-th degree is given by

$$
I_{E, V N D}\left(I_{A, V N D}, d_{v, i}, I_{E, D E T}\right)=\quad J\left(\sqrt{\left(d_{v, i}-1\right) J^{-1}\left(I_{A, V N D}\right)^{2}+\left[J^{-1}\left(I_{E, D E T}\right)\right]^{2}}\right)
$$

where the $J$-function is defined in [81] and is computed using the truncated-series representation presented in [82], and $I_{A, V N D}=I_{A, D E T}$. The VND curve describing the entire detector is given by

$$
I_{E, V N D}\left(I_{A, V N D}, I_{E, D E T}\right)=\sum_{i=1}^{D} b_{i} \cdot I_{E, V N D}\left(I_{A, V N D}, d_{v, i}, I_{E, D E T}\right)
$$

where $b_{i}=e_{v, i} / \sum_{i=1}^{D} e_{v, i}$ is the fraction of edges incident on variable nodes of degree $d_{v, i}$.

The CND curve is computed according to [38]

$$
I_{E, C N D}\left(I_{A, C N D}, d_{c}\right)=1-J\left(\sqrt{d_{c}-1} \cdot J^{-1}\left(1-I_{A, C N D}\right)\right)
$$

where $I_{A, C N D}$ is the mutual information between the check node inputs and the networkcoded bits. Note that the CND curve is independent of the particular variable node degree distribution $V$.

The curve fit for the VND and CND mutual information metrics corresponding to a particular degree distribution is performed by plotting the curves for a range of values of $\frac{E_{b}}{N_{0}}$ and noting the value in which the curves barely touch [38]. Specifically, the VND curve is plotted with $I_{A, V N D}$ on the horizontal axis and $I_{E, V N D}$ along the vertical. The CND curve is plotted with $I_{E, C N D}$ along the horizontal and $I_{A, C N D}$ along the vertical. The value of $\frac{E_{b}}{N_{0}}$ is varied from highest specified point to lowest, and the highest point for which the curves 
touch is defined as the EXIT threshold. Considering a particular relay receiver and channel configuration, the optimal variable node degree distribution is considered as the distribution having the lowest EXIT threshold.

\subsection{EXIT-Optimized LDPC Code Performance}

This section presents the error-rate performance of EXIT-optimized LDPC codes and the procedure used to generate the codes. Performance of the optimized codes is compared against standard codes. Several cases of modulation order and relay receiver CSI are considered. LDPC codes defined by the DVB-S2 and WiMAX standards are used as reference codes.

\subsubsection{Optimization Procedure}

The optimization procedure involves determining the detector EXIT characteristic through simulation for a chosen receiver configuration and channel model, and using this characteristic to generate the combined variable node/detector and check node characteristics over a range of SNR [38]. With regards to notation, [·] following a bold variable denotes a vector element, for instance, $\mathbf{Z}[k]$ denotes the $k$-th element of one-dimensional vector $\mathbf{Z}$ and $\mathbf{W}[i, k]$ denotes the $(i, k)$-th element of two-dimensional vector $\mathbf{W}$.

\section{Detector Characteristic}

The detector characteristic is computed via Monte Carlo simulation as follows

1. Select end node and relay frame size $N_{D}$, modulation order $M$, received channel state information (CSI or no CSI) and SNR value.

2. Select a discrete range of a-priori demodulator mutual information values, represented as the vector $\mathbf{I}_{A, D E T}[k]=k / B, 0 \leq k<B$. In all of our experiments, the value $B=100$ provided visibly smooth detector transfer characteristics. 
3. Simulate a frame of channel-corrupted relay-received symbols $\mathbf{Y}_{R}$ according to the model given in Section 3.2, having length $L_{D}=N_{D} / \mu$ and modulation order $M$.

4. For every value of $\mathbf{I}_{A, D E T}[k]$,

- Generate a vector of length $N_{D}$ a-priori LLRs $\mathbf{v}$ representing LDPC decoder feedback under the assumption that the feedback is conditionally Gaussian

$$
\mathbf{v}=(\mathbf{b}-1 / 2) \sigma_{k}^{2}+\mathbf{x} \sigma_{k}
$$

where $\sigma_{k}=J^{-1}\left(\mathbf{I}_{A, D E T}[k]\right)[38]$ and $\mathbf{x}$ is a length- $L$ vector of i.i.d. samples of a standard normal distribution.

- Compute the decoder extrinsic information $\mathbf{z}$ according to the receiver formulation provided in [30].

- Compute the simulated value of $\mathbf{I}_{E, D E T}[k]$ as $[58]$

$$
\mathbf{I}_{E, D E T}[k]=1-\frac{1}{L \log 2} \sum_{\ell=0}^{L} \max *\left(0, \mathbf{z}[\ell](-1)^{\mathbf{b}[\ell]}\right)
$$

- Numerically fit a third-order polynomial to the sampled detector characteristic using $\mathbf{I}_{A, D E T}$ as the abscissa and $\mathbf{I}_{E, D E T}$ as the ordinate, yielding coefficients $f_{0}, \ldots, f_{3}$

$$
\mathbf{I}_{E, D E T}[k]=f_{3} \mathbf{I}_{A, D E T}[k]^{3}+f_{2} \mathbf{I}_{A, D E T}[k]^{2}+f_{1} \mathbf{I}_{A, D E T}[k]+f_{0} .
$$

Define function $f_{D E T}(x)=f_{3} x^{3}+f_{2} x^{2}+f_{1} x+f_{0}$. This function approximates the detector characteristic and is used in the computation of the combined detector/decoder characteristic.

\section{Combined Detector/Decoder Characteristic}

The simulation procedure for generating the combined detector/decoder characteristics is as follows 
1. Select a discrete range of a-priori demodulator mutual information values, represented as the vector $\mathbf{I}_{A, V N D}[k]=k / B, 0 \leq k<B$. In all of our experiments, the value $B=100$ provided visibly smooth detector transfer characteristics.

2. For every value of detector a-priori mutual information $\mathbf{I}_{A, V N D}$,

- For every degree $d_{v, i}$ compute the a-priori information input to the detector $\mathbf{I}_{A, D E T}^{\prime}$ according to

$$
\mathbf{I}_{A, D E T}^{\prime}[i, k]=J\left(\sqrt{d_{v, i}} \cdot J^{-1}\left(\mathbf{I}_{A, V N D}[k]\right)\right) .
$$

- Compute $\mathbf{I}_{E, V N D}$ according to (4.4)

$$
\mathbf{I}_{E, V N D}[k]=\sum_{i=1}^{D} b_{i} \cdot J(\sqrt{G})
$$

where

$$
G=\left(d_{v, i}-1\right) J^{-1}\left(\mathbf{I}_{A, V N D}[k]\right)+\left[J^{-1}\left(f_{D E T}\left(\mathbf{I}_{A, D E T}^{\prime}[i, k]\right)\right)\right]^{2} .
$$

The check node characteristic is completely determined by the parameters of the code and is not dependent on the properties of the detector output. Noting that $I_{E, C N D}=I_{A, V N D}$ compute the a-priori CND characteristic according to (4.5)

$$
\mathbf{I}_{A, C N D}[k]=J\left(\frac{J^{-1}\left(1-\mathbf{I}_{E, C N D}[k]\right)}{\sqrt{d_{c}-1}}\right)
$$

\subsubsection{Optimization Results}

Optimization is performed for several cases of receiver configuration, channel state information, and code rate. Specifically, modulation orders $M=\{2,4,8\}$, Rayleigh fading with and without CSI, and AWGN are considered. The channel code parameters are taken from the DVB-S2 [70] and WiMAX [74], having codeword length $N=16200$ and rates $r_{D}=\{3 / 5,2 / 5\}$ for DVB-S2, and $N=2304$ and $r_{D}=2 / 3$ for WiMAX. Note that the WiMAX standard specifies two distinct parity check matrices for rate $2 / 3$ codes, denoted 
as 2/3A and 2/3B. In this work, case case $2 / 3 A$ is considered. For DVB-S2, at code rate $3 / 5$, the check node degree is $d_{c}=11$, and at rate $2 / 5, d_{c}=6$. In WiMAX, the check node degree is $d_{c}=10$.

The parity check matrix for the standard codes contain a sub-matrix satisfying the $e x-$ tended irregular repeat-accumulate (eIRA) constraint, simplifying encoding and decoding complexity [79]. The submatrices comprising $\mathbf{H}$ are

$$
\mathbf{H}=\left[\mathbf{H}_{1} \mid \mathbf{H}_{2}\right]
$$

where $\mathbf{H}_{2}$ has dual-diagonal eIRA structure with $(N-K)$ rows and $(N-K)$ columns. To preserve the complexity advantages of eIRA, we retain $\mathbf{H}_{2}$ exactly as specified by the standards and consider optimizing the variable node degrees specifying $\mathbf{H}_{1}$. Retaining $\mathbf{H}_{2}$ places constraints on $V$ such that codes based on DVB-S2 have $d_{v, 1}=2$, at rate $2 / 5$ have $o_{1}=9720$, and at rate $3 / 5$ have $o_{1}=6480$. Codes based on WiMAX have $d_{v, 1}=2, o_{1}=672$ and $d_{v, 2}=3, o_{2}=96$. All other degrees may be chosen freely.

The number of distinct variable node degrees is $D=3$, and the degrees considered are all combinations of the sets $d_{v, 1}=2, d_{v, 2} \in\{3,4, \ldots, 99\}$, and $d_{v, 3} \in\left\{d_{v, 2}+1, d_{v, 2}+2, \ldots, 98\right\}$ which satisfy the constraints for realizable codes described in Subsection 4.2.1. Considering WiMAX, the number of variable node degrees is $D=4$, and the degrees considered are all unique combinations of $d_{v, 1}=2, d_{v, 2}=3, d_{v, 3} \in\{3,4, \ldots, 99\}$, and $d_{v, 4} \in\left\{d_{v, 3}+1, d_{v, 3}+\right.$ $\left.2, \ldots, d_{v, 3}+97\right\}$.

For every receiver configuration and degree distribution $V$, the EXIT threshold is computed to discover the single variable node degree distribution having the best performance. For each distribution, the threshold is defined as the highest SNR value $\frac{E_{b}}{N_{0}}$ for which the VND and CND decoder EXIT curves touch. The best performing distribution is defined as minimizing this SNR value. An example is shown in Fig. 4.1. The parity check matrix $\mathbf{H}$ corresponding to a degree distribution $V$ is generated by the following heuristic (software for generating parity check matrices can be found at Dr. Radford Neal's home page http://www.cs.utoronto.ca/ radford/ftp/LDPC-2012-02-11/index.html). The 


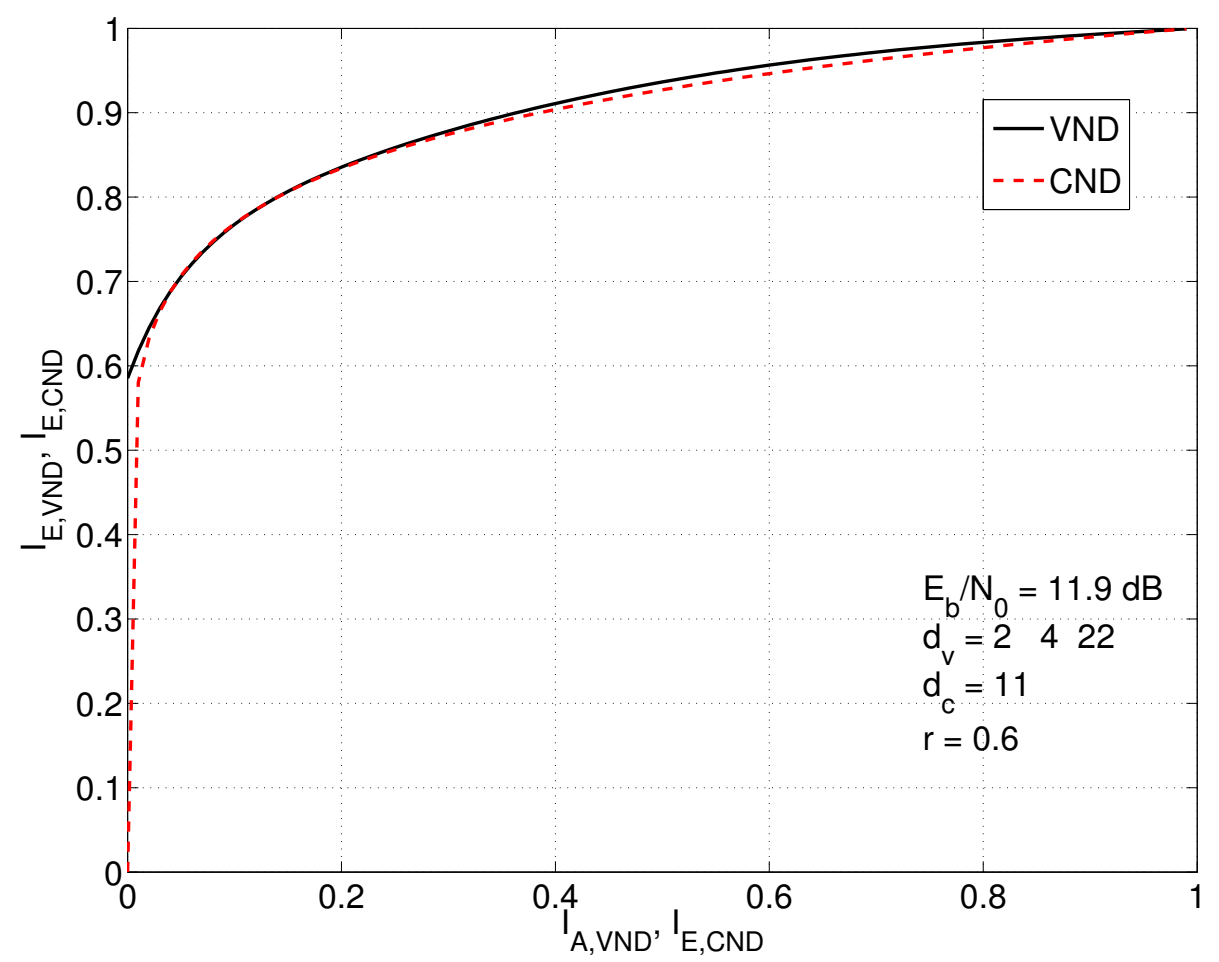

Figure 4.1: Example EXIT fit - DVB-S2 constraint

submatrix $\mathbf{H}_{1}$ having $N-K$ rows and $K$ columns is initialized to contain all zeros. For a particular degree distribution $V, \mathbf{H}_{1}$ will contain $o_{m}$ columns having $d_{v, m} 1$ 's, and $o_{n}$ columns containing $d_{v, n} 1$ 's, where $(m, n)=(2,3)$ in DVB-S2 and $(3,4)$ in WiMAX. The total number of 1's in $\mathbf{H}_{1}$ is then $T=d_{v, m} o_{m}+d_{v, n} o_{n}$. The pool of $T 1$ 's are assigned to rows as evenly as possible, with remainders assigned to rows uniformly at random. In the case that the column weights cannot be satisfied by the available pool of $T$ ones, 1 's are assigned at random to satisfy the column weights. Additional 1's are added to eliminate rows which have weight zero or one. The position for additional ones are selected uniformly at random from within the positions containing zeros. The resulting matrix $\mathbf{H}_{1}$ is concatenated with the eIRA matrix $\mathbf{H}_{2}$ to form the parity check matrix, as given by Eq. (4.13).

The optimization results for codes based on DVB-S2 are shown in Table 4.1 for code rate $r_{D}=3 / 5$ and Table 4.2 for $r_{D}=2 / 5$. At an operating BER of $10^{-4}$, the optimized codes outperform the standard codes for all receiver configurations and channels. At DVB-S2 
Table 4.1: Optimized LDPC variable node degrees based on DVB-S2 for code rate $r_{D}=3 / 5$. The SNRs required to reach a BER of $10^{-4}$ for optimized and standard codes are given in columns opt Opt. and Std. respectively. For all codes $d_{v, 1}=2, o_{1}=6480$ and $d_{c}=11$.

\begin{tabular}{|c|c|c|c|c|c|}
\hline \multirow[b]{3}{*}{ Channel } & \multirow[b]{3}{*}{ M } & \multicolumn{4}{|c|}{ Rate $r_{D}=3 / 5$} \\
\hline & & & \multicolumn{2}{|c|}{ Simulated (dB) } & \multirow{2}{*}{$\begin{array}{l}\text { Capacity } \\
\mathrm{CM}(\mathrm{dB})\end{array}$} \\
\hline & & $\mathrm{V}$ & Opt. & Std. & \\
\hline \multirow[t]{3}{*}{ AWGN } & 2 & 3:7290 15:2430 & 9.78 & 9.89 & 9.41 \\
\hline & 4 & $3: 8640$ 30:1080 & 6.65 & 6.99 & 6.29 \\
\hline & 8 & 3:8991 43:729 & 5.15 & 5.53 & 4.66 \\
\hline Rayleigh, & 2 & 4:8640 22:1080 & 17.4 & 17.8 & 16.2 \\
\hline Partial & 4 & $3: 826223: 1458$ & 13.0 & 13.8 & 11.7 \\
\hline CSI & 8 & $3: 864030: 1080$ & 10.5 & 11.6 & 9.40 \\
\hline \multirow{3}{*}{$\begin{array}{l}\text { Rayleigh, } \\
\text { No CSI }\end{array}$} & 2 & 3:7290 15:2430 & 17.4 & 17.9 & 16.4 \\
\hline & 4 & $3: 826223: 1458$ & 13.1 & 13.9 & 12.1 \\
\hline & 8 & $3: 864030: 1080$ & 10.9 & 11.8 & 9.83 \\
\hline
\end{tabular}

Table 4.2: Optimized LDPC variable node degrees based on DVB-S2 for code rate $r_{D}=2 / 5$. For all codes $d_{v, 1}=2, o_{1}=9720$ and $d_{c}=6$. See caption on Table 4.1 for a full description.

\begin{tabular}{|c|c|c|c|c|c|}
\hline \multirow[b]{3}{*}{ Channel } & \multirow[b]{3}{*}{ M } & \multicolumn{4}{|c|}{ Rate $r_{D}=2 / 5$} \\
\hline & & & Sim. & (dB) & Capacity, \\
\hline & & V & Opt. & Std. & $\mathrm{CM}(\mathrm{dB})$ \\
\hline \multirow[t]{3}{*}{ AWGN } & 2 & $3: 405011: 2430$ & 9.93 & 9.98 & 9.52 \\
\hline & 4 & 4:5760 22:720 & 7.09 & 7.17 & 6.57 \\
\hline & 8 & $3: 567027: 810$ & 5.54 & 5.86 & 5.07 \\
\hline \multirow{3}{*}{$\begin{array}{l}\text { Rayleigh, } \\
\text { Partial } \\
\text { CSI }\end{array}$} & 2 & $3: 486015: 1620$ & 14.8 & 15.0 & 13.9 \\
\hline & 4 & $4: 576022: 720$ & 11.0 & 11.3 & 9.94 \\
\hline & 8 & 3:4860 15:1620 & 9.18 & 9.44 & 8.02 \\
\hline \multirow{3}{*}{$\begin{array}{l}\text { Rayleigh, } \\
\text { No CSI }\end{array}$} & 2 & $3: 453613: 1944$ & 15.1 & 15.3 & 14.3 \\
\hline & 4 & 4:5832 24:648 & 11.4 & 11.6 & 10.4 \\
\hline & 8 & 4:5832 24:648 & 9.55 & 9.85 & 8.46 \\
\hline
\end{tabular}


Table 4.3: Optimized LDPC variable node degrees based on WiMAX for code rate $r_{D}=2 / 3$. The SNRs required to reach a BER of $10^{-4}$ for optimized and standard codes are given in columns opt Opt. and Std. respectively. For all codes $d_{v, 1}=2, o_{1}=672, d_{v, 2}=3, o_{2}=96$ and $d_{c}=10$.

\begin{tabular}{|c|c|c|c|c|c|}
\hline \multirow[b]{3}{*}{ Channel } & \multirow[b]{3}{*}{ M } & \multicolumn{4}{|c|}{ Rate $r_{D}=2 / 3$} \\
\hline & & & Sim. & $(\mathrm{dB})$ & Capacity, \\
\hline & & V & Opt. & Std. & $\mathrm{CM}(\mathrm{dB})$ \\
\hline Rayleigh, & 4 & 3:1376 12:160 & 14.7 & 15.2 & 12.4 \\
\hline Partial CSI & 8 & 3:1376 12:160 & 12.4 & 12.7 & 10.4 \\
\hline Rayleigh, & 4 & 3:1356 11:180 & 15.2 & 15.5 & 13 \\
\hline No CSI & 8 & 3:1392 13:144 & 12.7 & 13.1 & 10.8 \\
\hline
\end{tabular}

rate $r_{D}=3 / 5$, the optimal variable node degrees increase with modulation order, while at rate $r_{D}=2 / 5$ the degrees are more evenly distributed. The improvement of the optimized codes over standard is greater at rate $3 / 5$ than $2 / 5$. In all cases, the improvement over standard increases with modulation order. The performance gap of the optimized codes to CM capacity varies between approximately $0.5-1 \mathrm{~dB}$.

For WiMAX, optimization results are shown in Table 4.3. In all cases the difference between standard and optimized performance is between $0.3-0.5 \mathrm{~dB}$. The optimized codes lie from 2 to $3 \mathrm{~dB}$ away from capacity. Comparing to the results for the DVB-S2 codes, where a longer code length was used, we observe that performance gains from optimization are more evenly distributed, suggesting that longer block length provide more opportunity for optimization.

Simulated bit error rate performance for the optimized codes at rate $r_{D}=3 / 5$ is shown in Fig. 4.2. The BER was simulated in AWGN and Rayleigh fading with no CSI at modulation orders $M=\{2,4,8\}$. One hundred decoding iterations were performed, as a higher number of iterations conferred no additional benefit. The performance improvement of the optimized codes over the standard codes is nearly constant in the waterfall region.

Bit-error rate performance for the optimized codes based on WiMAX are shown in Figs. 4.3 and 4.4. For modulation order $M=4$ in Fig. 4.3, the partial CSI optimized code 


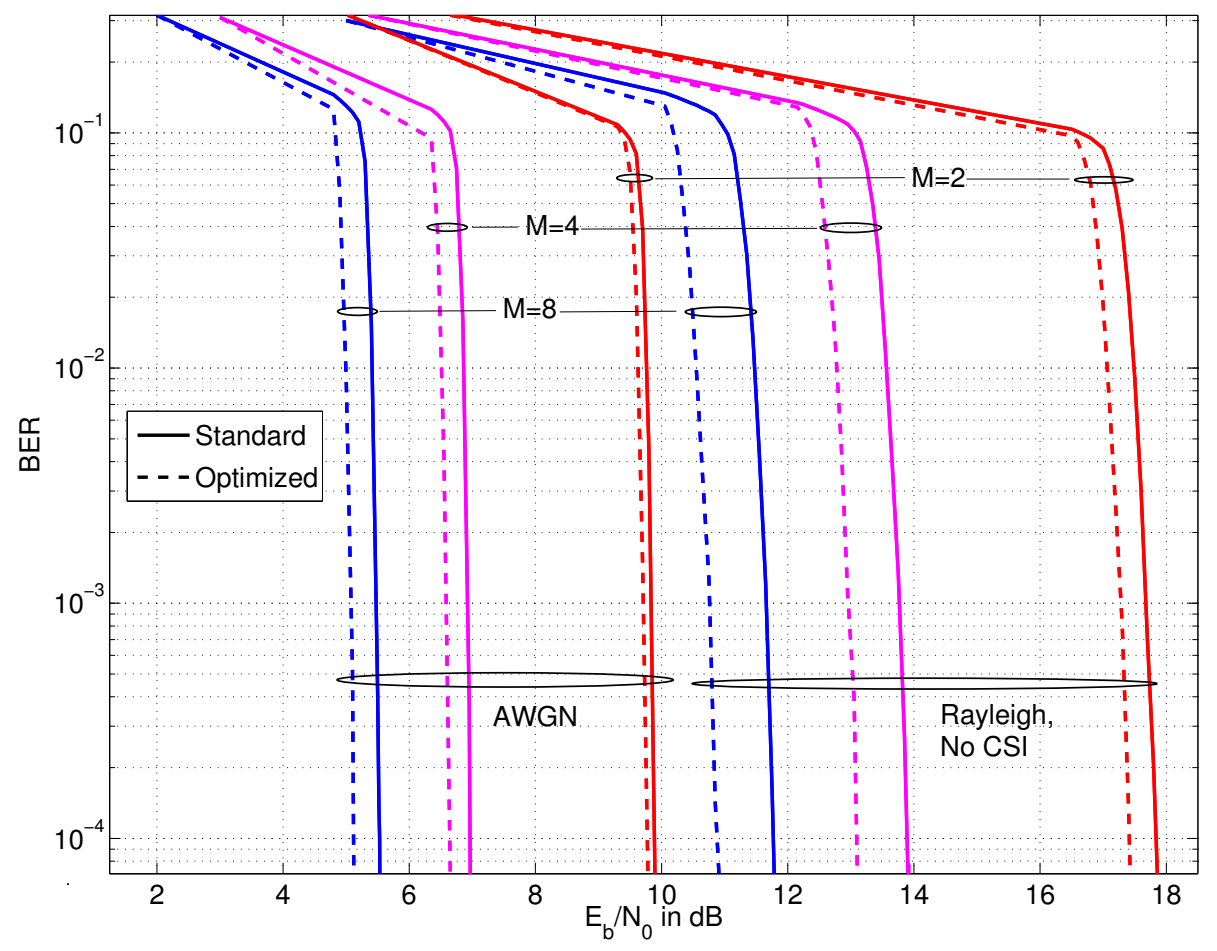

Figure 4.2: DVB-S2-inspired LDPC-coded BER performance at the relay using optimized channel codes for DNC. The channel code rate is $r_{D}=r_{M}=3 / 5$. Performance is simulated in AWGN and Rayleigh fading with no CSI at the relay. The frame length is $N_{D}=16200$ bits. FSK modulation orders $M=\{2,4,8\}$ are considered.

shows an improvement of about $0.4 \mathrm{~dB}$ over the standard code, while for the no CSI case, improvement is about $0.3 \mathrm{~dB}$. Both cases lie $3.2 \mathrm{~dB}$ from capacity. Considering $M=8$ in Fig. 4.4, under partial CSI the optimized code shows an improvement of about $0.4 \mathrm{~dB}$, while no CSI yields an improvement of $0.3 \mathrm{~dB}$. For both codes, the gap to capacity is about 2.8 $\mathrm{dB}$.

\subsection{Conclusion}

This chapter presented an optimization procedure for LDPC Tanner Graph variable node degree distribution in the physical-layer network coded two way relay channel multiple access stage. Optimized codes are generated under a variety of system configurations. The optimized codes outperform standard codes by margins from 0.3 to $1.2 \mathrm{~dB}$. 


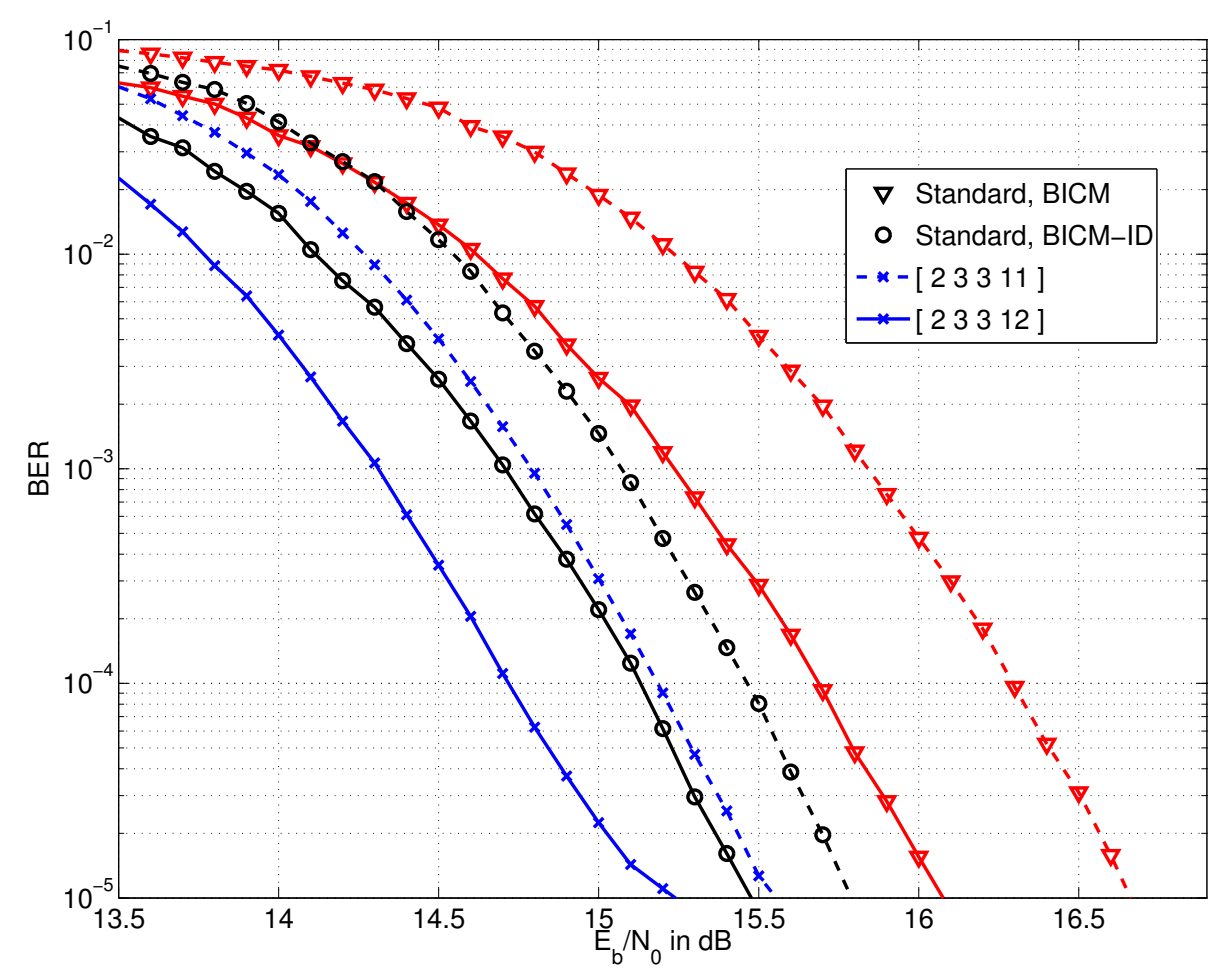

Figure 4.3: WiMAX-inspired LDPC-coded BER performance at the relay using optimized channel codes for DNC. The channel code rate, codeword length, and modulation order are $r_{D}=r_{M}=2 / 3, N=2304$ and $M=4$ respectively. A code from the WiMAX standard is simulated for comparison, denoted as "standard", while optimized codes are denoted by their degree distribution. Solid lines denote partial CSI at the relay, while dashed lines denote no CSI. 


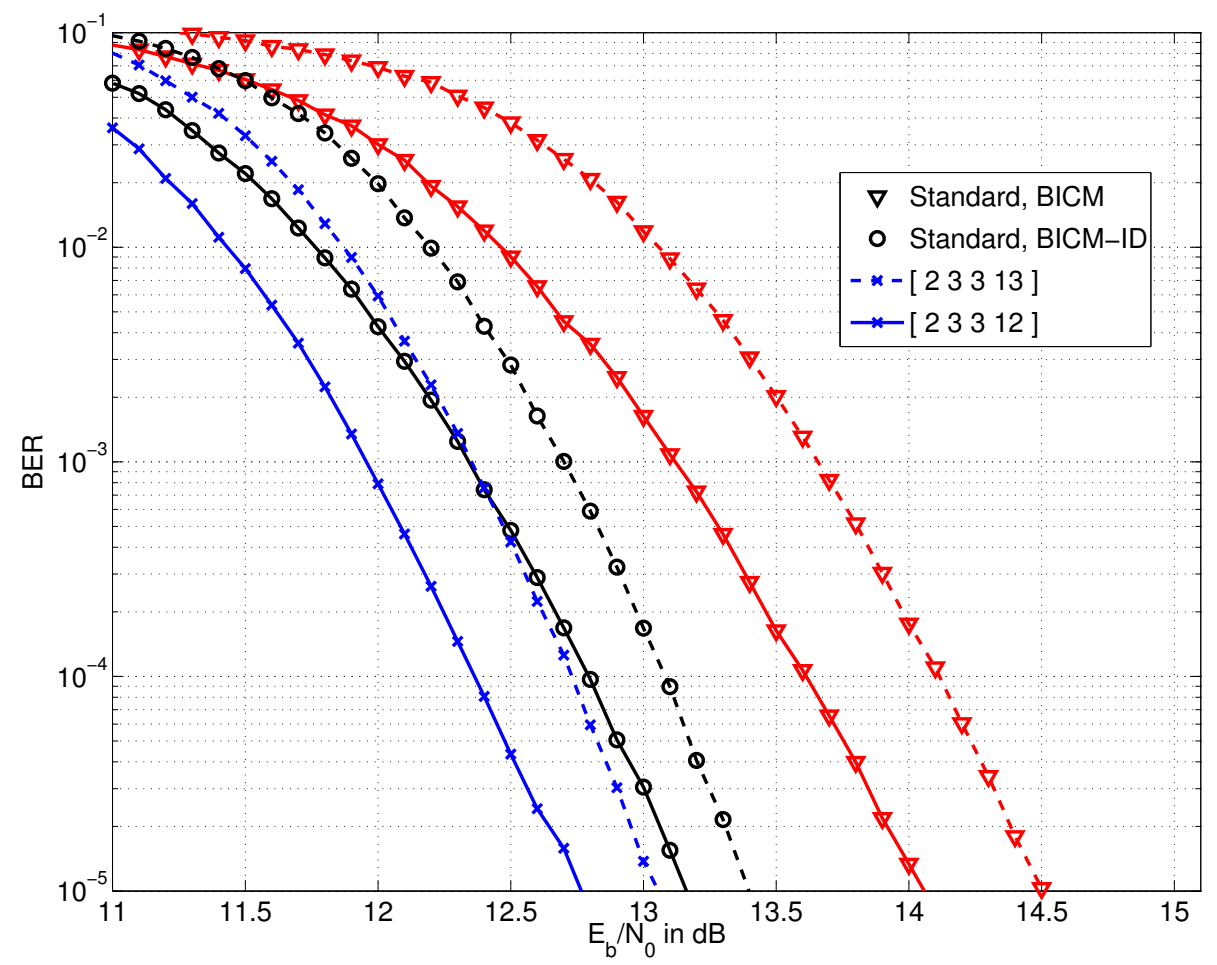

Figure 4.4: Error rate performance for EXIT-optimized LDPC codes at the TWRC relay during the multiple access phase. The modulation order is $M=8$. See the caption to Fig. 4.3 for remaining parameters. 


\section{Chapter 5}

\section{Iterative Noncoherent M-ary FSK System for ANC}

The focus of the previous chapters is the design of a demodulator for the digital networkcoded two-way relay channel. In this chapter, we consider the case where the relay amplifiesand-forwards the signals from the end nodes received during the multiple access stage, namely analog network coding. This chapter develops a noncoherent soft-output demodulator for the end nodes in the broadcast stage of the physical-layer network-coded two-way relay channel. Performance of the demodulator is investigated when coupled with LDPC channel coding.

\subsection{Introduction}

A primary distinction between PNC schemes is the forwarding technique applied by the relay [9]. In the case that the relay amplifies and forwards the signal received from the end nodes during the MA stage, the forwarding technique is termed PNC over an infinite field or analog network coding (ANC) [10]. When the relay demodulates and optionally performs channel decoding, the forwarding technique is referred to as PNC over a finite field or digital network coding (DNC) [45], as the relay detects and forwards information symbols over a discrete and finite set, such as an M-ary frequency-shift keyed (FSK) constellation.

In Chapter 3, a noncoherent FSK demodulator was developed for the relay in the MA 
stage that produced soft-outputs suitable for iterative channel decoding. In this chapter, a soft-output demodulator is derived for the end nodes assuming $A N C$ operation. Specifically, we develop a soft-output noncoherent M-FSK demodulator for the end nodes in the broadcast stage, where the relay amplifies and forwards the signals it receives from the end nodes during the MA stage. To our knowledge, this is the first soft-output demodulator for the ANC broadcast stage. The demodulator supports power-of-two modulation orders and produces log-likelihood ratios (LLRs) suitable for use with capacity approaching soft-decision decoding techniques. The performance of LDPC channel coding coupled with ANC is investigated in this chapter. As a noncoherent formulation, the demodulator is capable of operating without any knowledge of the channel and without phase synchronization between the end node and relay oscillators.

Previous work on ANC analyzes achievable transmission rates, compares with other TWRC protocols, and develops noncoherent receivers. An analysis of the achievable rates for ANC for a variety of network topologies is considered in [83]. Closed form expressions for the bit-error rate of noncoherent FSK in the passive RFID channel are derived in [84]. The passive RFID channel is analytically similar to the broadcast channel under ANC, as both consider a signal transmitted over two Rayleigh fading channels, an instance of double Rayleigh fading [85]. The relationship between bit error rate, transmission rate, and transmit power for the ANC TWRC is analyzed in [86], forming the basis for a rate and power adaptation scheme. A noncoherent receiver for the ANC TWRC using uncoded differential PSK modulation is developed in [87]. The BER of the receiver is derived and an optimal power allocation scheme is developed assuming constant fading coefficients per frame. A detailed capacity analysis for PNC and LNC as well as the four-step point-to-point protocol for the ANC relay assuming two-dimensional modulation is developed in [88].

The following organization is applied for the rest of the work. Section 5.2 describes the system model. Section 5.3 presents the ANC demodulator, developing the probability distribution of the symbols and bits received at the end nodes. Section 5.4 provides the simulation procedure and performance results used to investigate the performance of the 


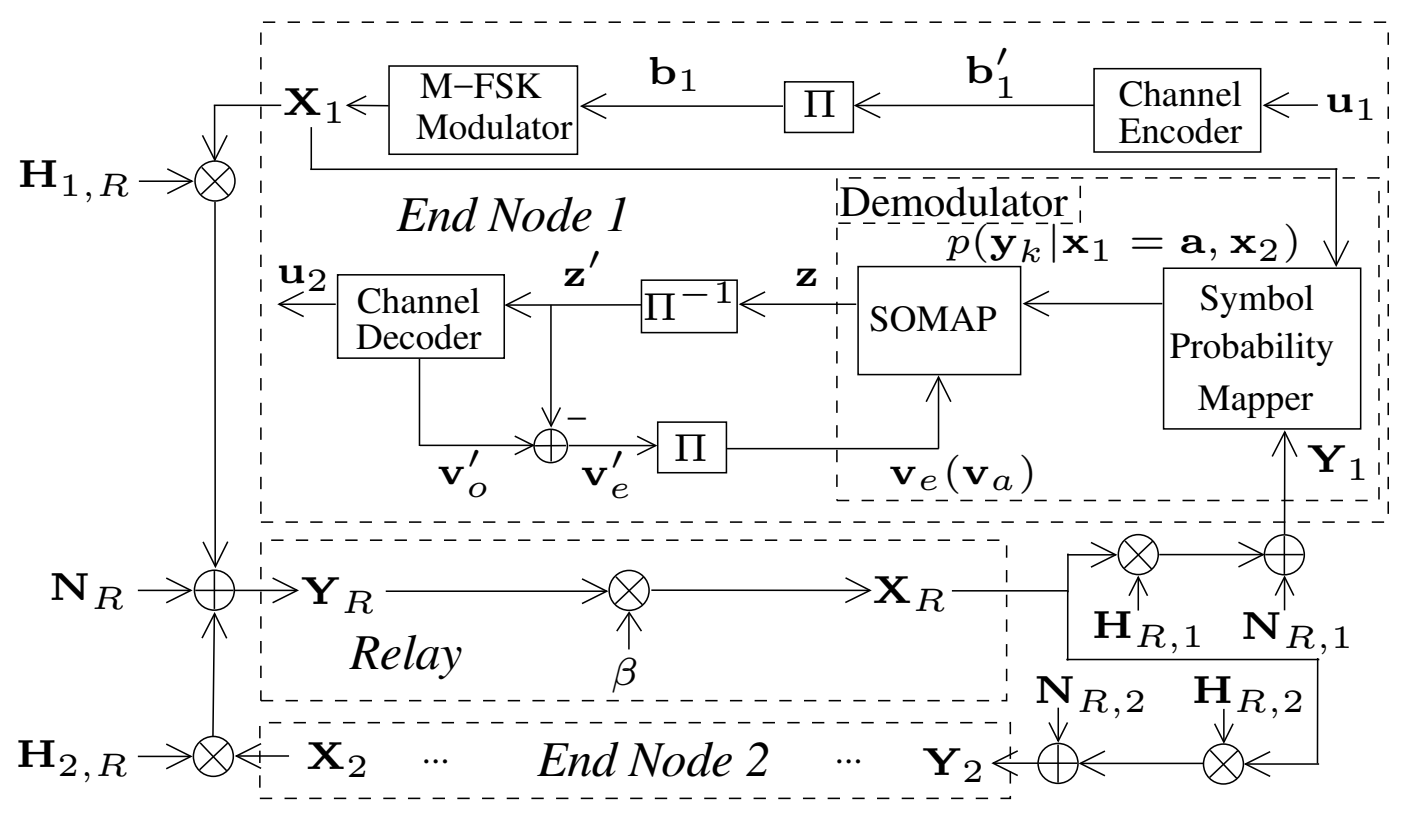

Figure 5.1: System Model - Analog Network Coded Two-way Relay Channel. The configuration of End Node 2 is identical to 1, and has been omitted from the figure.

developed demodulator. Concluding remarks are provided in Section 5.5.

\subsection{System Model}

This section describes the system model assumed for derivation and simulation of the ANC soft-output end node demodulator. Transmission by the end-nodes in the multiple access stage follows the model described in the introductory system model section, 1.3.1, and the assumed channel is described in the introductory channel model section, 1.3.2. Relay operation is described. End node reception using the developed demodulator with and without channel decoding is described. Symbol and frame synchronization is assumed throughout. The system model is shown in Fig. 5.1.

\subsubsection{Analog Network Coding at the Relay}

During the broadcast $(\mathrm{BC})$ stage, the purpose of the relay is to broadcast the frame of received symbols $\mathbf{Y}_{R}$ to the end nodes after scaling to satisfy the power constraint. Consider 
a single received symbol $\mathbf{y}_{k, R}$. The relay forms a symbol to transmit by scaling $\mathbf{y}_{k, R}$ as

$$
\begin{aligned}
\mathbf{x}_{k, R} & =\beta \mathbf{y}_{k, R} \\
& =\beta\left(h_{k, 1, R} \mathbf{x}_{k, 1}+h_{k, 2, R} \mathbf{x}_{k, 2}+\mathbf{n}_{k, R}\right)
\end{aligned}
$$

where $\mathbf{x}_{k, R}$ denotes the $k$-th symbol formed for transmission by the relay, and $\beta$ is a realvalued scaling factor which constrains the average transmitted energy. The relay forms a frame of symbols to broadcast to the end nodes as $\mathbf{X}_{R}=\left[\beta \mathbf{y}_{1, R}, \ldots, \beta \mathbf{y}_{N_{q}, R}\right]=\left[\mathbf{x}_{1, R}, \ldots, \mathbf{x}_{N_{q}, R}\right]$.

The value of the scaling factor $\beta$ which normalizes the transmitted energy depends on the statistics of the received symbols. Under noncoherent operation, the exact values of the fading coefficients $h_{k, i}$ are not known at the relay. It is assumed that the relay can estimate the statistics of the fading coefficients and additive noise. Specifically, the variances of the fading coefficients $\mathcal{E}_{i}$ and additive noise $N_{0}$ are assumed known through estimation.

Consider reception of a single symbol $\mathbf{y}_{k, R}$ at the relay. The total energy of the received symbol is

$$
\mathcal{E}_{k}=\sum_{m=0}^{M-1}\left|y_{m}\right|^{2}
$$

where $m$ denotes the $m$-th dimension of $\mathbf{y}_{k, R}$. The average energy received during a symbol period is computed as

$$
\begin{aligned}
\overline{\mathcal{E}}_{R} & =E\left[\sum_{m=0}^{M-1}\left|y_{m}\right|^{2}\right] \\
& =N_{0} M+\mathcal{E}_{1}+\mathcal{E}_{2}
\end{aligned}
$$

where it is assumed that the end nodes transmit all symbols with equal probability. The average energy transmitted by the relay is normalized to unity by setting the scaling factor as

$$
\beta=\frac{1}{\sqrt{N_{0} M+\mathcal{E}_{1}+\mathcal{E}_{2}}} .
$$

Since the scaling factor depends only on the statistics of the fading coefficients rather than the exact values, it is constant for a particular realization of the statistics. 


\subsubsection{End Node Reception}

The goal of reception at each end node is to detect the information bits transmitted by the opposite end node. During the BC stage, each end node receives the symbol frame broadcast by the relay after the frame has traversed a fading channel. Demodulation and optional channel decoding is performed to detect the desired information bits. Each end node knows the symbol frame it transmitted during the multiple access stage, and this information is used to compute the conditional probability of receiving particular symbols from the opposite node.

The frame received at end node $\mathcal{N}_{i}$ during the broadcast stage is

$$
\mathbf{Y}_{i}=\mathbf{X}_{R} \mathbf{H}_{R, i}+\mathbf{N}_{i}
$$

where $\mathbf{H}_{R, i}$ denotes the diagonal matrix of fading coefficients for the channel between the relay and end node $\mathcal{N}_{i}$, having dimensions $N_{q} \times N_{q}$, and $\mathbf{N}_{i}$ is an $M \times N_{q}$ noise matrix having the same distribution as $\mathbf{N}_{R}$. The channel gains forming the diagonal for matrix $\mathbf{H}_{R, i}$ are denoted by $h_{k, R, i}$ and are distributed as circularly symmetric complex jointly Gaussian $\mathcal{N}_{c}\left(0, \mathcal{E}_{R}\right)$. The matrix $\mathbf{H}_{R, i}$ takes value $h_{k, R, i}$ at row and column $(k, k)$ and 0 elsewhere.

The demodulator takes as input the symbols received from the relay $\mathbf{Y}_{R}$, the symbols transmitted by the end node during the multiple access stage $\mathbf{X}_{i}$, and a-priori probability (APP) information regarding the bits under detection $\mathbf{v}_{a}$. As output, the demodulator produces a-posteriori information regarding the bits under detection $\mathbf{z}$. The a-posteriori information is deinterleaved to produce $\mathbf{z}^{\prime}=\mathbf{z} \boldsymbol{\Pi}^{-1}$ and passed to the channel decoder. The decoder refines the estimate of $\mathbf{z}^{\prime}$, producing a-posteriori information $\mathbf{v}_{o}^{\prime}$. The decoder input is subtracted from the decoder output to produce extrinsic information $\mathbf{v}_{e}^{\prime}=\mathbf{v}_{o}^{\prime}-\mathbf{z}^{\prime}$ which is interleaved to produce $\mathbf{v}_{e}=\mathbf{v}_{e}^{\prime} \boldsymbol{\Pi}$ and returned to the demodulator. The decoder output becomes the demodulator a-priori input $\mathbf{v}_{a}=\mathbf{v}_{e}$.

The end nodes are assumed to know the average noise power $N_{0}$ and fading statistics in the form of variances $\mathcal{E}_{1}, \mathcal{E}_{2}$ and $\mathcal{E}_{R}$. This information can be obtained through a variety of techniques such as pilot symbols and control channels between the relay and end nodes. 
Knowledge of the noise power and fading statistics are assumed known in the formulation of the end node demodulator.

Formulation of the demodulator is described in Section 5.3. Details of the channel decoder have been described at length in the literature and will not be discussed in this work.

\subsection{Noncoherent End Node Demodulator}

This section develops the end node soft-output demodulator. The probability distribution of the symbols received at the end nodes is developed, followed by the model for iterative demodulation and decoding at the end node. Since demodulation is performed on a single symbol at a time, for the purpose of formulating the demodulator, we may drop the dependence on symbol period $k$ throughout to simplify the notation.

\subsubsection{End Node Received Symbol Distribution}

Consider a single received symbol at end node $\mathcal{N}_{i}$

$$
\begin{aligned}
\mathbf{y}_{i} & =h_{R, i} \mathbf{x}_{R}+\mathbf{n}_{i} \\
& =\beta h_{R, i}\left(h_{1, R} \mathbf{x}_{1}+h_{2, R} \mathbf{x}_{2}+\mathbf{n}_{R}\right)+\mathbf{n}_{i} .
\end{aligned}
$$

The term $\mathbf{x}_{R}$ is formed by the sum of three vectors, each having components which are circularly symmetric complex jointly Gaussian random variables, and all components are independent. Since the sum of complex jointly Gaussian random variables is also complex and jointly Gaussian, the components of $\mathbf{x}_{R}$ are distributed $\mathcal{N}_{c}\left(0, \sigma_{m}^{2}\right)$ where $\sigma_{m}^{2}$ is the variance of the $m$-th vector component $x_{m, R}$. The values of the variances depend on the symbols transmitted by the end nodes

$$
\sigma_{m}^{2}= \begin{cases}N_{0} & x_{m, 1}=0, x_{m, 2}=0 \\ N_{0}+\mathcal{E}_{1} & x_{m, 1}=1, x_{m, 2}=0 \\ N_{0}+\mathcal{E}_{2} & x_{m, 1}=0, x_{m, 2}=0 \\ N_{0}+\mathcal{E}_{1}+\mathcal{E}_{2} & x_{m, 1}=1, x_{m, 2}=1\end{cases}
$$


Now consider the distribution of the product of the fading coefficient $h_{R, i}$ and the symbol transmitted by the relay

$$
\begin{aligned}
\boldsymbol{\mu} & =h_{R, i} \mathbf{x}_{R} \\
& =\left[h_{R, i} x_{0, R}, \ldots, h_{R, i} x_{M-1, R}\right]^{T} . \\
& =\beta\left[\alpha_{0} e^{i \theta_{0}}, \ldots, \alpha_{M-1} e^{i \theta_{M-1}}\right]^{T} .
\end{aligned}
$$

Each component of $\boldsymbol{\mu}$ is the product of two independent circularly symmetric complex Gaussian random variables, which yields the complex double Gaussian distribution having pdf [89]

$$
p_{\mu_{m}}\left(\alpha_{m}, \theta_{m}\right)=\frac{2 \alpha_{m}}{\pi \mathcal{E}_{R} \sigma_{m}^{2}} K_{0}\left(\frac{2 \alpha_{m}}{\sqrt{\mathcal{E}_{R}} \sigma_{m}}\right)
$$

where $K_{0}(\cdot)$ is the modified Bessel function of the second kind [59].

We now derive the distribution of the received symbol which does not depend on knowledge of the fading amplitudes and phases. Denote the amplitudes of the components of $\boldsymbol{\mu}$ as $\boldsymbol{\alpha}=\left[\alpha_{0}, \ldots, \alpha_{M-1}\right]$ and the phases as $\boldsymbol{\theta}=\left[\theta_{0}, \ldots, \theta_{M-1}\right]$. The distribution of the received symbol conditioned on $\boldsymbol{\alpha}$ and $\boldsymbol{\theta}$ becomes

$$
\begin{aligned}
p(\mathbf{y} \mid \boldsymbol{\alpha}, \boldsymbol{\theta}) & =\left(\frac{1}{\pi N_{0}}\right)^{M} \exp \left[-\frac{\|\mathbf{y}-\boldsymbol{\mu}\|^{2}}{N_{0}}\right] \\
& =\left(\frac{1}{\pi N_{0}}\right)^{M} \prod_{m=0}^{M-1} \exp \left[-\frac{\left|y_{m}-\beta \alpha_{m} e^{i \theta_{m}}\right|^{2}}{N_{0}}\right] .
\end{aligned}
$$

Note that the joint distribution of the amplitude and phase given by (5.9) is the product of marginal distributions $p_{\mu_{m}}\left(\alpha_{m}, \theta_{m}\right)=p\left(\alpha_{m}\right) p\left(\theta_{m}\right)$, where

$$
p\left(\alpha_{m}\right)=\frac{4 \alpha_{m}}{\mathcal{E}_{R} \sigma_{m}^{2}} K_{0}\left(\frac{2 \alpha_{m}}{\sqrt{\mathcal{E}_{R}} \sigma_{m}}\right)
$$

and $p\left(\theta_{m}\right)=\frac{1}{2 \pi}, 0 \leq \theta_{m}<2 \pi$, thus, we may marginalize over the amplitude and phase separately.

Marginalizing over the phases yields 


$$
\begin{aligned}
& p(\mathbf{y} \mid \boldsymbol{\alpha})=\int_{0}^{2 \pi} p(\mathbf{y} \mid \boldsymbol{\alpha}, \boldsymbol{\theta}) p(\boldsymbol{\theta}) d \boldsymbol{\theta} \\
= & \left(\frac{1}{\pi N_{0}}\right)^{M} \prod_{m=0}^{M-1} \int_{0}^{2 \pi} \exp \left[-\frac{\left|y_{m}-\beta \alpha_{m} e^{i \theta_{m}}\right|^{2}}{N_{0}}\right] \frac{1}{2 \pi} d \theta_{m} \\
= & \left(\frac{1}{\pi N_{0}}\right)^{M} \exp \left[-\frac{\sum_{m=0}^{M-1}\left|y_{m}\right|^{2}}{N_{0}}\right] \prod_{m=0}^{M-1} \exp \left[-\frac{\beta^{2} \alpha_{m}^{2}}{N_{0}}\right] I_{0}\left(\frac{2 \beta \alpha_{m}\left|y_{m}\right|}{N_{0}}\right)
\end{aligned}
$$

where $I_{0}(\cdot)$ is the modified Bessel function of the first kind [59].

Marginalizing over the amplitudes yields

$$
\begin{aligned}
& p(\mathbf{y})=\int_{0}^{\infty} p(\mathbf{y} \mid \boldsymbol{\alpha}) p(\boldsymbol{\alpha}) d \boldsymbol{\alpha} \\
= & \left(\frac{1}{\pi N_{0}}\right)^{M} \exp \left[-\frac{\sum_{m=0}^{M-1}\left|y_{m}\right|^{2}}{N_{0}}\right]\left(\frac{4}{\mathcal{E}_{R}}\right)^{M} \times \ldots \\
& \prod_{m=0}^{M-1} \frac{1}{\sigma_{m}^{2}} \int_{0}^{\infty} \exp \left[-\frac{\beta^{2} \alpha_{m}^{2}}{N_{0}}\right] I_{0}\left(\frac{2 \beta \alpha_{m}\left|y_{m}\right|}{N_{0}}\right) \alpha_{m} K_{0}\left(\frac{2 \alpha_{m}}{\sqrt{\mathcal{E}_{R}} \sigma_{m}}\right) d \alpha_{m}
\end{aligned}
$$

For the purpose of performing the integration given by (5.13), we may neglect the terms outside the integral for a moment, yielding

$$
\int_{0}^{\infty} \alpha_{m} \exp \left[-\frac{\beta^{2} \alpha_{m}^{2}}{N_{0}}\right] I_{0}\left(\frac{2 \beta \alpha_{m}\left|y_{m}\right|}{N_{0}}\right) K_{0}\left(\frac{2 \alpha_{m}}{\sqrt{\mathcal{E}_{R}} \sigma_{m}}\right) d \alpha_{m} .
$$

To perform the integration, we represent the modified Bessel function of the first kind as a series [59]

$$
I_{0}(x)=\sum_{n=0}^{\infty} \frac{x^{2 n}}{4^{n} n !^{2}}
$$

After substituting (5.15) into (5.14), the integral becomes

$$
\int_{0}^{\infty} \alpha_{m} \exp \left[-\frac{\beta^{2} \alpha_{m}^{2}}{N_{0}}\right] \sum_{n=0}^{\infty} \frac{\left(c_{1} \alpha_{m}\right)^{2 n}}{4^{n} n !^{2}} K_{0}\left(\frac{2 \alpha_{m}}{\sqrt{\mathcal{E}_{R}} \sigma_{m}}\right) d \alpha_{m}
$$

where $c_{1}=2 \beta\left|y_{m}\right| / N_{0}$. Factoring out constants with respect to the integration and rearranging, (5.16) becomes

$$
\sum_{n=0}^{\infty} \frac{c_{1}^{2 n}}{4^{n} n !^{2}} \int_{0}^{\infty} \alpha_{m}^{2 n+1} \exp \left[-\frac{\beta^{2} \alpha_{m}^{2}}{N_{0}}\right] K_{0}\left(\frac{2 \alpha_{m}}{\sqrt{\mathcal{E}_{R}} \sigma_{m}}\right) d \alpha_{m}
$$


Define $c_{2}=\beta^{2} / N_{0}$. We then make the change of variable $u=c_{2} \alpha_{m}^{2}$ and $d u=2 c_{2} \alpha_{m} d \alpha_{m}$. Then $\alpha_{m}=\sqrt{u / c_{2}}$ and $d \alpha_{m}=d u /\left(2 c_{2} \alpha_{m}\right)$. Substituting the change of variable into (5.17)

$$
\frac{1}{2} \sum_{n=0}^{\infty} \frac{c_{1}^{2 n}}{4^{n} n !^{2} c_{2}^{n+1}} \int_{0}^{\infty} u^{n} \exp (-u) K_{0}\left(c_{4} \sqrt{u}\right) d u
$$

where $c_{4}=2 /\left(\mathcal{E}_{R} \sigma_{m}^{2} \sqrt{c_{2}}\right)$. Applying integration formula (6.643-3) in [90], (5.18) becomes

$$
\frac{\exp \left(c_{4}^{2} / 8\right)}{c_{4}} \sum_{n=0}^{\infty} \frac{c_{1}^{2 n}}{4^{n}} W_{-(n+1 / 2), 0}\left(\frac{c_{4}^{2}}{4}\right)
$$

where $W_{a, b}(x)$ is the Whittaker-W function [59] having parameters $a$ and $b$ and argument $x$.

Substituting the result of integration (5.19) into (5.13) yields the PDF of the received symbol having no dependence on the channel state

$$
\begin{array}{r}
p(\mathbf{y})=\left(\frac{1}{\pi \sqrt{N_{0}} \sqrt{\mathcal{E}_{R}} \beta}\right)^{M} \exp \left[\sum_{m=0}^{M-1}\left(-\frac{\left|y_{m}\right|^{2}}{N_{0}}+\frac{N_{0}}{2 \mathcal{E}_{R} \sigma_{m}^{2} \beta^{2}}\right)\right] \times \ldots \\
\prod_{m=0}^{M-1} \frac{1}{\sigma_{m}} \sum_{n=0}^{\infty}\left(\frac{\left|y_{m}\right|^{2}}{N_{0}}\right)^{n} W_{-(n+1 / 2), 0}\left(\frac{N_{0}}{\mathcal{E}_{R} \sigma_{m}^{2} \beta^{2}}\right) .
\end{array}
$$

This expression is suitable for performing noncoherent soft output detection at the end nodes. The PDF contains an infinite summation, which is truncated for implementation.

\subsubsection{Iterative Demodulation and Decoding}

The end node demodulator maps the symbols received from the relay during the broadcast stage to log-likelihood ratios of the bits transmitted by the opposite end node. In the following section, without loss of generality, consider reception at end node $\mathcal{N}_{1}$, where the goal is to recover the information sequence $\mathbf{u}_{2}$ transmitted by $\mathcal{N}_{2}$. Iterative decoding is performed whereby the channel decoder feeds information back to the demodulator, which refines the bit estimates and sends them back to the channel decoder. A hard decision is made on the bits after the specified iteration count has been reached.

The soft mapper (SOMAP) [66] operates on a symbol-by-symbol basis, transforming symbol probabilities $p\left(\mathbf{y} \mid \mathbf{x}_{1}=\mathbf{a}, \mathbf{x}_{2}\right)$ to the set of $\mu$ log-likelihood ratios associated with 
each bit mapped to symbol $\mathbf{x}_{2}$. The term $\mathbf{a}$ is the symbol transmitted by the receiving end node during the symbol period under consideration, which is available, since the end node knows the data that it transmitted. The SOMAP takes as input the symbol probabilities and a-priori information fed back from the channel decoder about the bits mapped to the symbols $\mathbf{v}_{a}$. The SOMAP produces a-posteriori log-likelihood ratios of the bits mapped to the channel symbols $\mathbf{z}$. On the first iteration, no decoding has been performed, and the bit probabilities are assumed equally likely, yielding $\mathbf{v}_{a}=\mathbf{0}$.

The a-priori log-likelihood ratio of the $m$-th bit mapped to input symbol $\mathbf{x}_{2}$ is

$$
v_{k}=\log \frac{P\left(u_{k}=1 ; I\right)}{P\left(u_{k}=0 ; I\right)}, 0 \leq k \leq \mu-1
$$

The a-posteriori SOMAP output is the log-likelihood ratio of the $k$-th bit mapped to $\mathbf{x}_{2}$

$$
z_{k}=\log \frac{P\left(u_{k}=1 ; O\right)}{P\left(u_{k}=0 ; O\right)}, 0 \leq k \leq \mu-1
$$

The SOMAP input is transformed to output according to

$$
P\left(u_{k}=\ell ; O\right)=\sum_{\mathbf{x}_{2}: u_{k}=\ell} p\left(\mathbf{y} \mid \mathbf{x}_{1}=\mathbf{a}, \mathbf{x}_{2}\right) \prod_{\substack{j=0 \\ j \neq m}}^{\mu-1} P\left(u_{j} ; I\right)
$$

Substituting (5.21) into the expression for output (5.23),

$$
P\left(u_{k}=\ell ; O\right)=\sum_{\mathbf{x}_{2}: u_{k}=\ell} p\left(\mathbf{y} \mid \mathbf{x}_{1}=\mathbf{a}, \mathbf{x}_{2}\right) \prod_{\substack{j=0 \\ j \neq m}}^{\mu-1} \frac{e^{u_{j} v_{j}}}{1+e^{v_{j}}}
$$

The SOMAP out log-likelihood ratio may be found by combining (5.24) and (5.22):

$$
z_{k}=\log \frac{\sum_{\mathbf{x}_{2}: u_{k}=1} p\left(\mathbf{y} \mid \mathbf{x}_{1}=\mathbf{a}, \mathbf{x}_{2}\right) \prod_{\substack{j=0 \\ j \neq m}}^{\mu-1} e^{u_{j} v_{j}}}{\sum_{\mathbf{x}_{2}: u_{k}=0} p\left(\mathbf{y} \mid \mathbf{x}_{1}=\mathbf{a}, \mathbf{x}_{2}\right) \prod_{\substack{j=0 \\ j \neq m}}^{\mu-1} e^{u_{j} v_{j}}}
$$

where the term $\left(1+e^{v_{j}}\right)$ cancels in the ratio. When implementing (5.25), simplification using the max-star operator provides numerical stability. The max-star operator is defined as 


$$
\max _{i} *\left\{x_{i}\right\}=\log \left\{\sum_{i} e^{x_{i}}\right\}
$$

where the binary max-star operator is $\max *(x, y)=\max (x, y)+\log \left(1+e^{-|x-y|}\right)$ and multiple arguments are recursive. For example, in the case of three arguments, max-star becomes $\max *(x, y, z)=\max *(x, \max *(y, z))$. Applying the max-star operator to (5.25)

$$
z_{k}=\max _{\mathbf{x}_{2}: u_{k}=1}\left[\log p\left(\mathbf{y} \mid \mathbf{x}_{1}=\mathbf{a}, \mathbf{x}_{2}\right)+\sum_{\substack{j=0 \\ j \neq k}}^{\mu-1} u_{j} v_{j}\right]-\max _{\mathbf{x}_{2}: u_{k}=0}\left[\log p\left(\mathbf{y} \mid \mathbf{x}_{1}=\mathbf{a}, \mathbf{x}_{2}\right)+\sum_{\substack{j=0 \\ j \neq k}}^{\mu-1} u_{j} v_{j}\right] .
$$

A non-iterative demodulator does not use decoder feedback, and is implemented using (5.27) setting all $v_{j}=0$.

The term $\log p\left(\mathbf{y} \mid \mathbf{x}_{1}=\mathbf{a}, \mathbf{x}_{2}\right)$ in (5.27) is computed by taking the logarithm of (5.20), yielding

$$
\begin{aligned}
& \log p\left(\mathbf{y} \mid \mathbf{x}_{1}, \mathbf{x}_{2}\right)=\sum_{m=0}^{M-1}\left[\frac{N_{0}}{2 \mathcal{E}_{R} \sigma_{m}^{2} \beta^{2}}-\log \sigma_{m}\right]+\ldots \\
& \sum_{m=0}^{M-1} \max _{0 \leq n \leq N_{t}}\left[2 n \log \left|y_{m}\right|-n \log N_{0}+\log W_{-(n+1 / 2), 0}\left(\frac{N_{0}}{\sigma_{R}^{2} \sigma_{m}^{2} \beta^{2}}\right)\right] .
\end{aligned}
$$

where the infinite series has been truncated to a finite number of terms $N_{t}$. Note that the following terms in (5.20)

$$
\left(\frac{1}{\pi \sqrt{N_{0}} \sqrt{\mathcal{E}_{R}} \beta}\right)^{M} \exp \left[\sum_{m=0}^{M-1}\left(-\frac{\left|y_{m}\right|^{2}}{N_{0}}\right)\right]
$$

cancel in the ratio given by (5.27), and are not included in (5.28). Demodulator performance as a function of the truncation length $N_{t}$ is investigated in Section 5.4.

\subsection{Demodulator Performance}

This section presents Monte Carlo simulated error rate performance for the demodulator derived in Section 5.3. Error rate performance is simulated using different values of 
modulation order, demodulator summation terms, with and without channel coding, with and without decoder feedback to the demodulator (BICM vs BICM-ID) and signal-to-noise ratio. Both end nodes and the relay transmit each each symbol with unit energy, making the variance of the fading coefficients $\mathcal{E}_{1}=\mathcal{E}_{2}=\mathcal{E}_{R}=1$. The channel code considered is the LDPC code defined by the DVB-S2 standard [70].

\subsubsection{Error Rate Performance}

The results of error rate simulation are presented in this subsection. All uncoded simulations use frame size $K=2048$ bits. Coded simulations use the DVB-S2 LDPC code with codeword length $L=16200$ and rate $K / L=1 / 2$. All coded simulations apply 100 decoding iterations. When no information is fed back from the decoder to the demodulator (BICM), all decoding iterations are performed by the decoder. When information is fed back from the decoder to the demodulator (BICM-ID), a single channel decoder iteration is performed for every iteration between the decoder and the demodulator. BICM-ID is performed for modulation orders $M>2$, as there it provides no benefit for $M=2$. The FSK modulation orders considered are $M=\{2,4,8\}$. Computation of the infinite series in the expression for received symbol probabilities (5.28) is truncated to finite values $N_{t}=\{5,10,15,20,50\}$. For all simulations, enough trials are run to yield smooth error rate curves.

Uncoded end node error rate performance as a function of modulation order and number of demodulator infinite series terms is shown in Fig. 5.2. For both modulation orders $M=2$ and $M=4$ and $N_{t}<50$, a behavior is observed where detection fails completely after a particular SNR threshold is reached. At $N_{t}=\{5,15,25\}$, the error threshold occurs at error rates $\approx 10^{-1}, \approx 10^{-3}$, and $\approx 10^{-4}$ respectively. For $N_{t}=50$, no threshold is observed for the error rates considered. These results suggest that a minimum number of terms must be computed to operate at a particular error rate.

Channel coded error rate performance as a function of modulation order and number of infinite series terms is shown in Fig. 5.3. In all cases, BICM with no decoder to demodulator feedback was used. As in the uncoded case, performance is affected by the number of infinite 


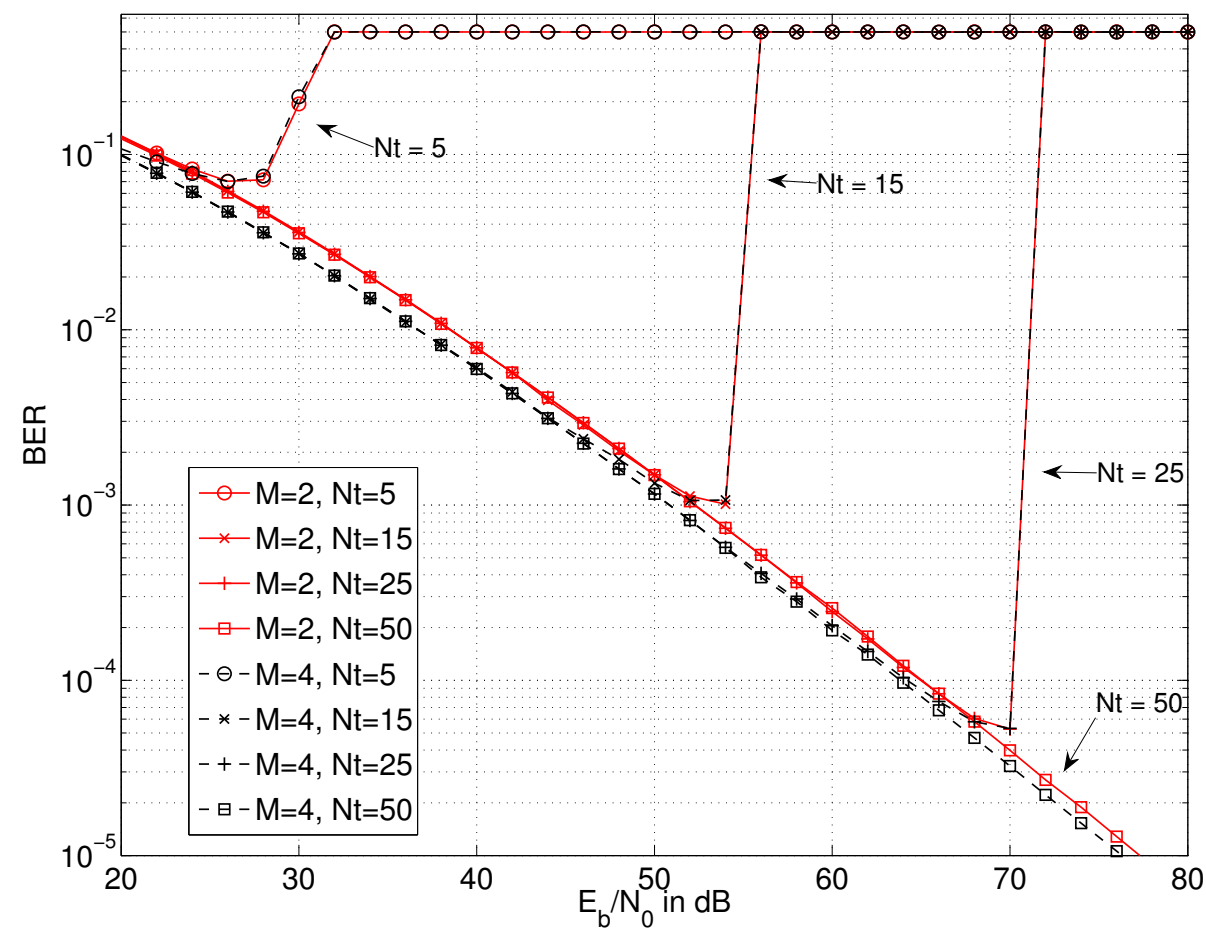

Figure 5.2: Bit error rate performance with no channel coding at the end node in the twoway relay channel broadcast stage under Rayleigh fading. The modulation orders considered are $M=\{2,4\}$. The number of demodulator infinite series terms considered are $N_{t}=$ $\{5,15,25,50\}$.

series terms computed $N_{t}$, however, an error threshold is only observed for the case $N_{t}=5$. When channel coding is applied, the number of infinite series terms affects the location of the decoding waterfall region. For modulation order $M=4$, the worst performing waterfall at $N_{t}=10$ is about $0.9 \mathrm{~dB}$ worse than the best performing waterfall at $N_{t}=50$. The same difference is observed for modulation order $M=8$. In the coded case, generally, fewer infinite series terms are required for successful decoding than in the uncoded case, suggesting a tradeoff between demodulation and decoding complexity.

Channel coded error rate performance as a function of modulation order and decoder feedback is shown in Fig. 5.4. All codes are simulated using $N_{t}=50$ infinite series terms at the demodulator. The purpose of this experiment is to investigate the performance benefit yielded by information feedback from decoder to demodulator, and the absolute performance 


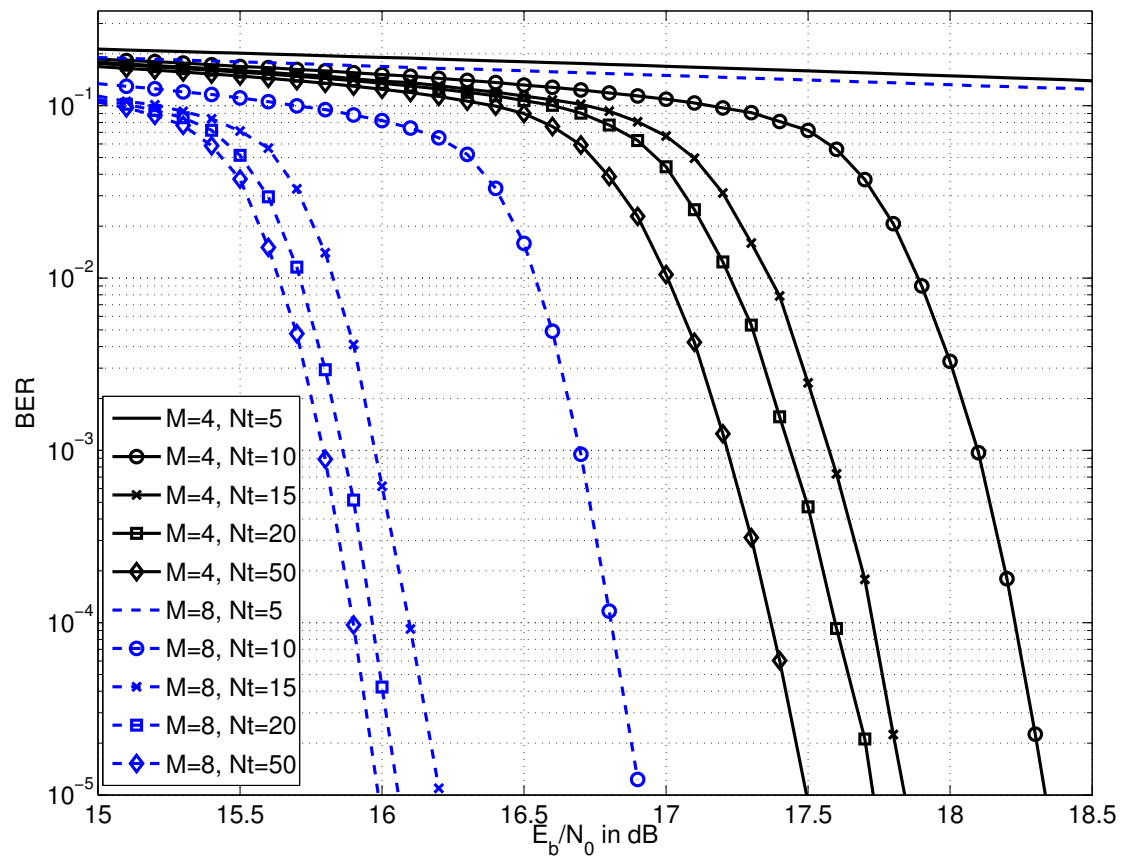

Figure 5.3: LDPC-coded bit error rate performance at as a function of demodulator infinite series terms. The LDPC code parameters are codeword length $L=16200$ and rate $r_{S}=1 / 2$. All simulations use BICM decoding.

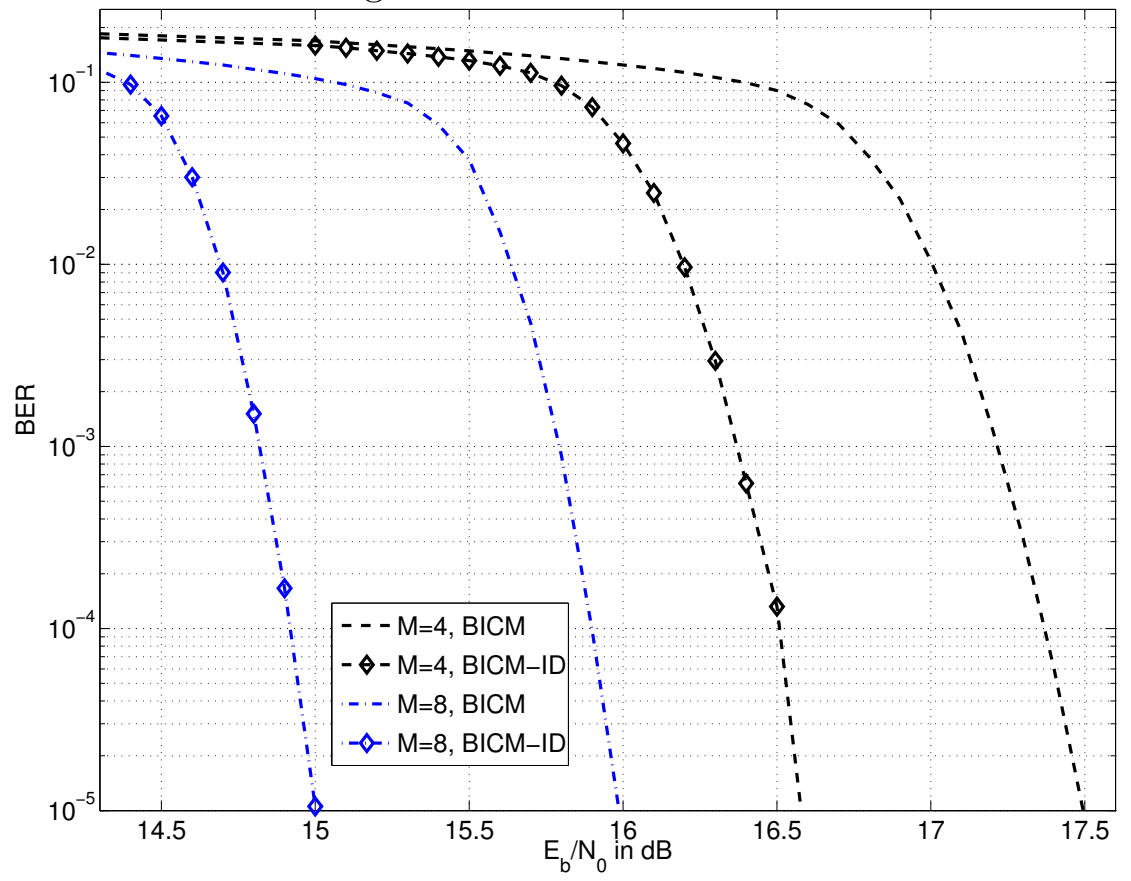

Figure 5.4: LDPC-coded bit error rate performance as a function of decoder feedback (BICM vs BICM-ID). The LDPC code parameters are codeword length $L=16200$ and rate $r_{S}=1 / 2$. For all simulation $N_{t}=50$. 
difference between modulation orders $M=4$ and $M=8$. For modulation order $M=4$, the BICM-ID exhibits a performance gain of $0.9 \mathrm{~dB}$ over BICM. For $M=8$, BICM-ID exhibits a gain of $1 \mathrm{~dB}$. BICM for $M=8$ outperforms BICM for $M=4$ by approximately $1.5 \mathrm{~dB}$.

\subsection{Conclusion}

This chapter developed a noncoherent soft output FSK demodulator the end nodes in the analog network-coded two-way relay channel under Rayleigh fading. The demodulator supports power of two modulation orders and iteration with the channel decoder. The demodulator formulation contains an infinite series which must be truncated for practical receiver implementation. It is demonstrated the bit error rate performance is sensitive to the infinite series truncation length. An exact characterization of the convergence of the demodulator as well as a closed form expression are left as future work. 


\section{Chapter 6}

\section{Other Contributions}

The purpose of this chapter is to describe contributions that deviate from one or more of the fundamental assumptions made in the previous contributions described in this dissertation. In Section 6.1, we relax the assumption that the end node and relay oscillators are synchronized in frequency, referred to as frequency offset. We investigate the performance loss resulting from frequency offset and formulate a demodulator to mitigate the loss. Section 6.2 considers a multiple-access stage containing any number of sources, where all previous contributions only considered two. The complexity of demodulation grows exponentially in the number of sources, and a technique is applied to manage this complexity.

\subsection{Physical-layer Network Coding Using FSK Modulation with Frequency Offset}

In this section, we analyze the effects of frequency offset on the oscillators at the nodes in physical-layer network-coded two-way relay channel. Specifically, we consider the case where all nodes used oscillators having arbitrary offsets on the frequencies used to generate each FSK tone, and the effect on bit-error rate performance at the relay during the multiple-access stage. A detection rule is derived that compensates for frequency offset, and performance is investigated with and without compensation. 


\subsubsection{Introduction}

The goal of the work presented in this section is to extend the previous by relaxing the assumption that the oscillators generating FSK tone frequencies at the end nodes are perfectly synchronized, and develop a noncoherent relay detector which compensates for the lack of synchronization.

Crystal oscillators are commonly used to generate reference frequencies for signals such as carriers and correlator reference signals. The frequencies generated by crystal oscillators are offset from ideal specifications due to manufacturing imperfections, ambient conditions such as temperature and radiation level, as well as aging of the oscillator [91]. In this work, oscillator offsets are modeled as real values added to the frequencies of the FSK tones generated by the end nodes which are constant for all symbol periods.

The effect of oscillator offset on a conventional single-source, single-destination link has been well studied for FSK systems, comprising performance analysis, simulation, algorithms to estimate offset at the receiver, and techniques to synchronize the clocks of network nodes. In an AWGN channel, oscillator offset imposes an error-rate floor on FSK modulation when the receiver does not compensate, as shown by the performance analyses in [92] and [93]. An analysis of the BER performance of 4-FSK as a function of frequency offset is given in [94]. The effect of carrier frequency on an OFDM-based PNC system is analyzed and investigated in a hardware testbed in [95]. A frequency offset estimation algorithm at the receiver may be applied to correct the offset of the received signal prior to demodulation and noncoherent detection of CPFSK, as described by [96]. Frequency offset estimates may also be used to adjust the receiver filter frequencies prior to demodulation [97]. In networks containing multiple nodes simultaneously transmitting to a single receiver, synchronization may be performed by synchronizing the clocks of all nodes to a single master [98]. In this work, our goal is to avoid the complexity of clock synchronization techniques by deriving a detection rule which compensates for frequency offset.

The specific contributions of this work are

1) A vector channel model for the received signal at the relay in the two-way relay network 


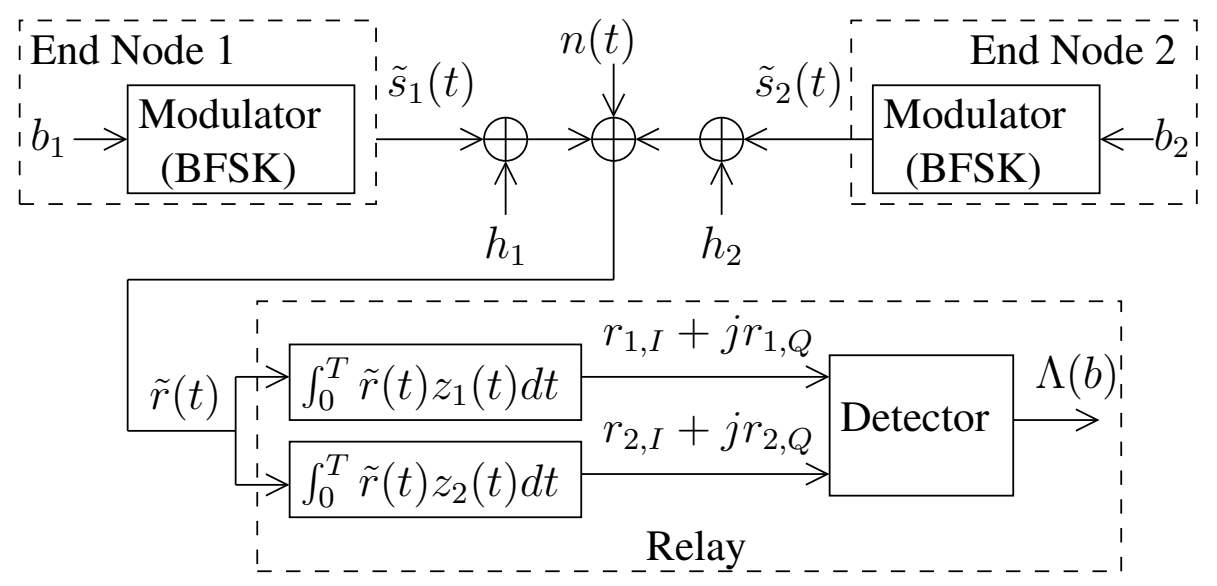

Figure 6.1: Baseband Transmission Model

under digital network coding using frequency-shift keyed modulation with tone offsets and frequency-flat Rayleigh fading.

2) A noncoherent detector for digital network-coded FSK at the relay in the threeterminal relay network which compensates for frequency offset at the end nodes. The detectors exhibit reasonable performance for frequency offsets on the order of a few hundredths of the FSK tone spacing. The output of the detector is a soft output appropriate for use with channel codes such as turbo codes.

\subsubsection{System Model}

The two-way relay channel using the digital network coding protocol is modeled assuming binary frequency-shift keyed modulation (BFSK) and frequency-flat, slowly-varying Rayleigh fading channels, as shown in Fig. 6.1. During a single symbol epoch, the end nodes $\mathcal{N}_{i}, i \in$ $\{1,2\}$ each modulate a bit $b_{i} \in\{0,1\}$ which is mapped to a BFSK symbol and transmitted to the relay. The bit may be channel coded or uncoded. The signals traverse independent fading channels, corrupting the amplitude and phase of each signal. The relay receives the electromagnetic sum of the faded signals plus noise.

Each end node transmits symbols having period $T$. The set of BFSK symbols at end 
node $\mathcal{N}_{i}$ during a single symbol epoch is represented in continuous time as

$$
s_{i}(t)=\operatorname{Re}\left[\tilde{s}_{i}(t) e^{j 2 \pi f_{c} t}\right], \quad 0<t<T
$$

where $f_{c}$ is the carrier frequency, $t$ is time, and the complex baseband transmitted signal is

$$
\tilde{s}_{i}(t)=\sqrt{\frac{2}{T}} e^{j 2 \pi\left(b_{i} \Delta_{f}+d_{i}\right) t}
$$

where $b_{i}$ is the bit transmitted by end node $\mathcal{N}_{i}, \Delta_{f}$ is the FSK tone frequency spacing with value $1 / T$, and $d_{i}$ is the frequency offset between end node $\mathcal{N}_{i}$ and the relay.

Oscillator frequency offset at the end nodes is modeled as a constant, continuous value, representing an oscillator operating in static ambient conditions. Denote FSK symbols 1 and 2 as the tones generated when the information bit $b_{i}$ takes values 0 and 1 , respectively.

The signal received at the relay during a single symbol epoch is

$$
r(t)=R e\left[\left(\sum_{i=1}^{2} h_{i} \tilde{s}_{i}(t)+n(t)\right) e^{j 2 \pi f_{c} t}\right]
$$

where $h_{i}=\alpha_{i} e^{j \theta_{i}}$ is the complex Gaussian channel gain between the relay and end nodes $\mathcal{N}_{i}$ with variance $\sigma_{h}^{2}$ per complex dimension, $\alpha_{i}$ is a fading coefficient distributed as Rayleigh $(\sigma)$, and $\theta_{i}$ is the phase offset uniformly distributed as $U(0,2 \pi)$, and $n(t)$ is circularly-symmetric complex Gaussian noise. The exact values of $\alpha_{i}$ and $\theta_{i}$ are not known at the relay receiver, however, the variance of per complex dimension $\sigma_{h}^{2}$ is known. The complex received signal translated to baseband is

$$
\tilde{r}(t)=\sum_{i=1}^{2} h_{i} \tilde{s}_{i}(t)+n(t)
$$

\subsubsection{Detection Rule}

In this section, a detection rule compensating for frequency offset is derived. The output at the relay matched filters is analyzed, and frequency offset is modeled as a multiplicative effect on the energy received in each filter dimension. The log-likelihood ratio (LLR) of receiving each network-coded bit is derived. A detection rule is formulated using the LLR. 


\section{Matched Filter Output Analysis}

In this section, the form of the signal samples at the output of the relay matched filters are derived. The received signal is translated to baseband and correlated against reference signals representing the in-phase and quadrature components of the FSK tones transmitted by the end nodes. One sample per symbol is assumed. The frequency of the oscillator at the relay is defined as $f_{c}$.

The samples at the output of the matched filters, considering a single symbol interval, are defined as

$$
\mathbf{r}=\left[\begin{array}{c}
r_{1, I}+j r_{1, Q} \\
r_{2, I}+j r_{2, Q}
\end{array}\right]
$$

where

$$
\begin{aligned}
r_{m, I}+j r_{m, Q} & =\int_{0}^{T} \tilde{r}(t) z_{m}(t) d t, m \in\{1,2\} \\
& =\int_{0}^{T}\left[\sum_{i=1}^{2} h_{i} \tilde{s}_{i}(t)+n(t)\right] z_{m}(t) d t
\end{aligned}
$$

where $r_{m, I}$ and $r_{m, Q}$ are the in-phase and quadrature component of the $m$-th correlator sample, $\tilde{r}(t)$ is the baseband received signal, and $z_{m}(t)$ is the $m$-th correlator reference signal, defined as

$$
z_{m}(t)=\sqrt{\frac{2}{T}} e^{-j 2 \pi \Delta_{f}(m-1) t}, m \in\{1,2\}
$$

Substituting the expressions for the low-pass equivalent signals transmitted by the end nodes (6.2) and the expressions for the reference signals (6.7) into the expression for the correlator samples (6.6) and simplifying yields the final form for the correlator samples

$$
\begin{aligned}
& r_{m, I}+j r_{m, Q}= \\
& \sum_{i=1}^{2} h_{i}\left[\frac{\sin A_{i, m}}{A_{i, m}}-j \frac{\cos A_{i, m}-1}{A_{i, m}}\right]+n_{m, I}+j n_{m, Q}
\end{aligned}
$$


where

$$
A_{i, m}=2 \pi\left\{\left[b_{i}-(m-1)\right]+d_{i} / \Delta_{f}\right\}
$$

and $n_{m, I}$ and $n_{m, Q}$ are independent Gaussian random variables having mean 0 and variance $\sigma_{n}^{2}=N_{0} / 2$.

The matched filter output statistics $r_{m, I}$ and $r_{m, Q}$ are thus Gaussian random variables having variance $N_{0} / 2$ and means which depend on the bits transmitted, the fading coefficients, and the magnitude of the frequency offsets at each end node.

\section{Vector Channel Model Under Oscillator Offset}

This section defines a vector channel model for the signal received at the relay. It is shown that oscillator offset can be modeled as a multiplicative effect with respect to the transmitted symbol and effects of the channel. The vector notation is used to express the relay matched filter samples.

Define the following vector random variable representing the bits transmitted by each end node

$$
\mathbf{v}_{k}=\left[b_{1} b_{2}\right], \quad k \in\{1,2,3,4\}
$$

with the following mapping of events

$$
\begin{aligned}
& \mathbf{v}_{1}=\left[\begin{array}{ll}
0 & 0
\end{array}\right] \quad \mathbf{v}_{3}=\left[\begin{array}{ll}
0 & 1
\end{array}\right] \\
& \mathbf{v}_{2}=\left[\begin{array}{ll}
1 & 1
\end{array}\right] \quad \mathbf{v}_{4}=\left[\begin{array}{ll}
1 & 0
\end{array}\right]
\end{aligned}
$$

The bit-to-symbol mapping used by the end nodes is defined as follows. Denote the symbols transmitted by end nodes $\mathcal{N}_{1}$ and $\mathcal{N}_{2}$ as the vectors $\mathbf{s}_{1}$ and $\mathbf{s}_{2}$ respectively, with the following mapping of bits to symbols

$$
\mathbf{s}_{i}=\left\{\begin{array}{lll}
{\left[\begin{array}{lll}
1 & 0
\end{array}\right]^{T}} & b_{i}=0 \\
{\left[\begin{array}{lll}
0 & 1
\end{array}\right]^{T}} & b_{i}=1
\end{array} i \in\{1,2\}\right.
$$


Define the following matrix containing symbols $\mathbf{s}_{1}$ and $\mathbf{s}_{2}$

$$
\mathbf{S}=\left[\mathbf{s}_{1} \mathbf{s}_{2}\right]
$$

with value chosen from the set of symbol matrices

$$
\begin{aligned}
& \mathbf{S}_{1}=\left[\begin{array}{ll}
1 & 1 \\
0 & 0
\end{array}\right] \mathbf{S}_{3}=\left[\begin{array}{ll}
1 & 0 \\
0 & 1
\end{array}\right] \\
& \mathbf{S}_{2}=\left[\begin{array}{ll}
0 & 0 \\
1 & 1
\end{array}\right] \mathbf{S}_{4}=\left[\begin{array}{ll}
0 & 1 \\
1 & 0
\end{array}\right]
\end{aligned}
$$

Note that in (6.8) the effects of oscillator offset on the matched filter samples are multiplicative. This suggests that oscillator offset can be incorporated into the vector channel model as a matrix multiplication. Denote the multiplicative effect of oscillator offset at end node $\mathcal{N}_{i}$ given by $(6.8)$ as

$$
O_{i}\left[b_{i}, m\right]=\left[\frac{\sin A_{i, m}}{A_{i, m}}-j \frac{\cos A_{i, m}-1}{A_{i, m}}\right] i \in\{1,2\}
$$

where $A_{i, m}$ is given by (6.9), and the particular values of $b_{i}$ and $m$ substituted into $A_{i, m}$ are denoted by $\left[b_{i}, m\right]$.

Gathering the offset terms at end node $\mathcal{N}_{i}$ as a matrix

$$
\mathbf{O}_{i}=\left[\begin{array}{cc}
O_{i}[0,1] & O_{i}[1,1] \\
O_{i}[0,2] & O_{i}[1,2]
\end{array}\right] i \in\{1,2\}
$$

the channel statistics at the output of the relay demodulator considering a single symbol interval is expressed in vector form as

$$
\mathbf{r}=h_{1} \mathbf{O}_{1} \mathbf{s}_{1}+h_{2} \mathbf{O}_{2} \mathbf{s}_{2}+\mathbf{n}
$$

where $\mathbf{n}=\left[\begin{array}{ll}n_{1} & n_{2}\end{array}\right]^{T}$, and $n_{1}$ and $n_{2}$ are complex jointly Gaussian random variables.

\section{Detection Rule}

The goal of this section is to derive the optimal detection rule for the network-coded bit at the relay. A general probabilistic model of the network coding operation is developed, the detection rule is derived assuming no knowledge of channel state at the relay. 
To detect the network-coded bit, the relay detector computes the log-likelihood ratio of the network-coded bit $b$

$$
\Lambda(b)=\log \frac{P(b=1)}{P(b=0)}=\log \frac{P\left(b_{1} \oplus b_{2}=1\right)}{P\left(b_{1} \oplus b_{2}=0\right)}
$$

and the log-likelihood ratio of the network-coded bit given by (6.18) is expressed in terms of $\mathbf{v}_{i}$

$$
\begin{aligned}
\Lambda(b) & =\log \frac{P\left(\mathbf{v}_{1} \cup \mathbf{v}_{2} \mid \mathbf{r}\right)}{P\left(\mathbf{v}_{3} \cup \mathbf{v}_{4} \mid \mathbf{r}\right)} \\
& =\log \frac{P\left(\mathbf{S}=\mathbf{S}_{1} \mid \mathbf{r}\right)+P\left(\mathbf{S}=\mathbf{S}_{2} \mid \mathbf{r}\right)}{P\left(\mathbf{S}=\mathbf{S}_{3} \mid \mathbf{r}\right)+P\left(\mathbf{S}=\mathbf{S}_{4} \mid \mathbf{r}\right)} \\
& =\log \frac{p\left(\mathbf{r} \mid \mathbf{S}=\mathbf{S}_{1}\right)+p\left(\mathbf{r} \mid \mathbf{S}=\mathbf{S}_{2}\right)}{p\left(\mathbf{r} \mid \mathbf{S}=\mathbf{S}_{3}\right)+p\left(\mathbf{r} \mid \mathbf{S}=\mathbf{S}_{4}\right)}
\end{aligned}
$$

where the second line follows from noting that the symbol pairs $\mathbf{S}_{i}$ are mutually exclusive, and the third line follows from applying Bayes' rule to $P\left(\mathbf{S}_{i} \mid \mathbf{r}\right)$ and assuming that the end node bits $b_{1}$ and $b_{2}$ are independent, and distributed equally likely.

Consider transmission of symbol pair $\mathbf{S}$ by the end nodes. The received signal at the relay may be written as

$$
\mathbf{r}=\left[\begin{array}{l}
h_{1} O_{1}\left[b_{1}, 1\right]+h_{2} O_{2}\left[b_{2}, 1\right]+n_{1} \\
h_{1} O_{1}\left[b_{1}, 2\right]+h_{2} O_{2}\left[b_{2}, 2\right]+n_{2}
\end{array}\right]
$$

where $O_{1}\left[b_{1}, 1\right], O_{2}\left[b_{2}, 1\right] . O_{1}\left[b_{1}, 2\right] . O_{2}\left[b_{2}, 2\right]$ are elements of the offset matrices in $(6.16)$ selected according to the symbol pair transmitted by the end nodes.

Let $O_{i}\left[b_{i}, m\right]=\beta_{i, m}+\gamma_{i, m}$. Express the fading coefficients as $h_{1}=h_{1, I}+j h_{1, Q}$ and $h_{2}=h_{2, I}+j h_{2, Q}$, where $h_{1, I}, h_{1, Q}, h_{2, I}, h_{2, Q}$ are i.i.d. $\mathcal{N}\left(0, \sigma_{h}^{2}\right)$. Substituting the above definitions into (6.20) and simplifying,

$$
\mathbf{r}=\left[\begin{array}{c}
\beta_{1,1} h_{1, I}-\gamma_{1,1} h_{1, Q}+\beta_{2,1} h_{2, I}-\gamma_{2,1} h_{2, Q}+n_{1, I} \\
+j\left(\gamma_{1,1} h_{1, I}-\beta_{1,1} h_{1, Q}+\gamma_{2,1} h_{2, I}-\beta_{2,1} h_{2, Q}+n_{1, Q}\right) \\
\beta_{1,2} h_{1, I}-\gamma_{1,2} h_{1, Q}+\beta_{2,2} h_{2, I}-\gamma_{2,2} h_{2, Q}+n_{2, I} \\
+j\left(\gamma_{1,2} h_{1, I}-\beta_{1,2} h_{1, Q}+\gamma_{2,2} h_{2, I}-\beta_{2,2} h_{2, Q}+n_{2, Q}\right)
\end{array}\right]
$$


where the additive noise term $\mathbf{n}$ has been expressed in in-phase and quadrature form as $\mathbf{n}=\left[n_{1, I}+j n_{1, Q} n_{2, I}+j n_{2, Q}\right]^{T}$.

The components of the demodulator outputs are formed from the sum of scaled, independent, zero-mean complex Gaussian random variables, thus, the components are complex jointly Gaussian random variables, and completely described by their mean vector and covariance matrix. The covariance matrix assuming transmission of symbol pair $\mathbf{S}=\mathbf{S}_{i}$ is given by

$$
\mathbf{K}=E\left[\mathbf{r r}^{H}\right]
$$

The $\{l, k\}$-th element of $\mathbf{K}_{i}$ is given by

$$
K_{l, k}=E\left[r_{l} r_{k}\right] l, k \in\{1,2\}
$$

Enumerating all values of $K_{l, k}$

$$
\begin{aligned}
& K_{1,1}=2\left[\left(\beta_{1,1}^{2}+\gamma_{1,1}^{2}+\beta_{2,1}^{2}+\gamma_{2,1}^{2}\right) \sigma_{h}^{2}+\sigma_{n}^{2}\right] \\
& K_{1,2}=2\left(\beta_{1,1} \beta_{1,2}+\gamma_{1,1} \gamma_{1,2}+\beta_{2,1} \beta_{2,2}+\gamma_{2,1} \gamma_{2,2}\right) \sigma_{h}^{2} \\
& K_{2,1}=2\left(\beta_{1,1} \beta_{1,2}+\gamma_{1,1} \gamma_{1,2}+\beta_{2,1} \beta_{2,2}+\gamma_{2,1} \gamma_{2,2}\right) \sigma_{h}^{2} \\
& K_{2,2}=2\left[\left(\beta_{1,2}^{2}+\gamma_{1,2}^{2}+\beta_{2,2}^{2}+\gamma_{2,2}^{2}\right) \sigma_{h}^{2}+\sigma_{n}^{2}\right]
\end{aligned}
$$

The distribution of the demodulator outputs is thus given by

$$
p\left(\mathbf{r} \mid \mathbf{S}_{i}\right)=\frac{1}{(2 \pi)^{2}|\mathbf{K}|} \exp \left(-\mathbf{r}^{H} \mathbf{K}^{-1} \mathbf{r}\right)
$$

where $\mathbf{K}^{-1}$ is the inverse of the covariance matrix $\mathbf{K}$, and $|\mathbf{K}|$ is the determinant. The log-likelihood ratio of the network-coded bit $b$ under noncoherent operation is computed by substituting the conditional distribution (6.25) into the general expression for the loglikelihood ratio (6.19), where

$$
\log \left[p\left(\mathbf{r} \mid \mathbf{S}=\mathbf{S}_{i}\right)\right]=-2 \log 2 \pi-\log |\mathbf{K}|-\mathbf{r}^{H} \mathbf{K}^{-1} \mathbf{r}
$$

is the logarithm of (6.25). 


\subsubsection{Simulation Results}

This section presents the simulated error rate performance of the noncoherent detector presented in Section 6.1.3. For all simulations, the frequency offset is normalized with respect to the tone spacing $\Delta f$. Assume that the symbol rate transmitted by the end nodes is proportional to tone spacing. The simulated frequency offset values are several hundredths of a tone spacing, representing the case in which offset is a modest fraction of symbol rate. Error rate performance is given for several values of frequency offset at the end nodes with and without an error-correcting channel code. The channel code used in simulation is the UMTS turbo code [62]. A full description of the application of turbo codes to the network considered in this work is given in [29]. Performance of the detector which incorporates oscillator offset knowledge is compared against the receiver rule which does not explicitly model offset.

The error rate performance considering offset between the relay and a single end node is shown in Fig. 6.2. This scenario models the case in which the oscillator at the relay is frequency locked to the oscillator at end node 1 . The detection rule that does not incorporate offset reaches a minimum error rate and then degrades in performance as a function of SNR. The detection rule incorporating offset outperforms the rule which does not, however, the error rate encounters an error floor as SNR increases. Further analysis is required to determine the reason that the detection rule incorporating offset reaches an error floor. The implication of the simulation results is that offsets less than 0.04 fractions of a tone spacing permit error rates lower than $10^{-2}$.

The performance of the noncoherent detection rule under an offset between both end nodes and the relay is shown in Fig. 6.3. This scenario models the case in which the oscillator frequency at the relay is not locked to either end node. The numerical results imply that performance is dominated by the relative offset between the oscillators at end nodes 1 and 2, as performance in the case of $d_{1}=d_{2}=0.04$ is approximately equal to the case of $d_{1}=d_{2}=0$ shown in Fig. 6.2. Absolute values of offset lead to an energy loss which is insignificant in the offset values considered in simulation. 


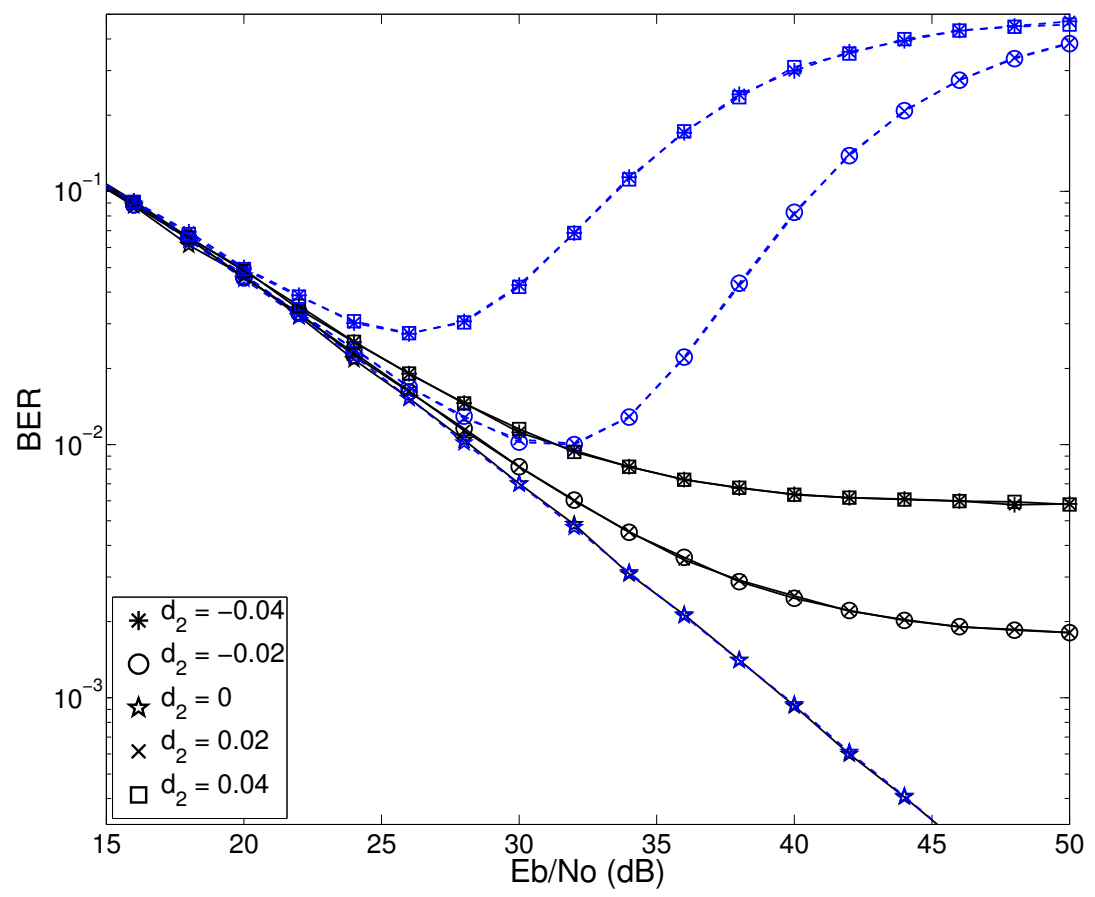

Figure 6.2: Simulated performance of noncoherent detection rules under oscillator offset. Blue, dashed lines denote the detection rule which does not model offset, while black, solid lines denote the detection rule which does model offset. Offset $d_{1}=0$ for all cases.

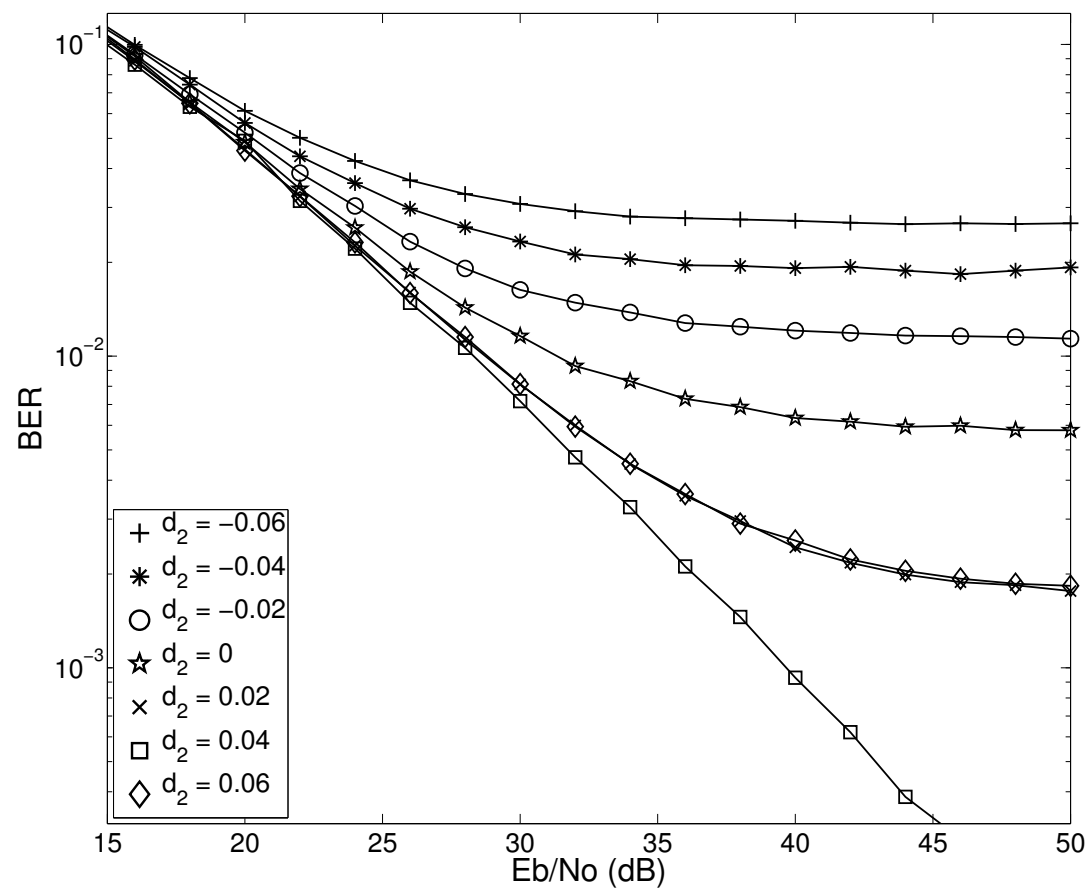

Figure 6.3: Simulated performance of noncoherent detection rule incorporating frequency offset assuming nonzero offsets at both end nodes. Offset $d_{1}=0.04$ for all cases. 
Table 6.1: Example values of Oscillator Offset

\begin{tabular}{|c|c|c|c|}
\hline $\begin{array}{c}\text { Carrier } \\
\text { Frequency } \\
(\mathrm{GHz})\end{array}$ & $\begin{array}{c}\text { Tone } \\
\text { Spacing, } \Delta f \\
(\mathrm{kHz})\end{array}$ & $\begin{array}{c}\text { Offset, } d \\
(\mathrm{kHz})\end{array}$ & $\begin{array}{c}\text { Offset, } \frac{d}{\Delta f} \\
\text { (normalized) }\end{array}$ \\
\hline 1.0 & 250 & 10 & 0.02 \\
\hline 2.4 & 2400 & 48 & 0.04 \\
\hline 4.9 & 3200 & 98 & 0.03 \\
\hline
\end{tabular}

Performance of the noncoherent detection rule when a turbo code is applied to protect the information bits at both end nodes is shown in Fig. 6.4. The rate of the turbo code is chosen to illustrate that the detection rule which models offset is capable of achieving lower error rates for particular values of SNR than the rule which does not model offset. Consider the simulation case of $d_{2}=0.06$. At an SNR of $36 \mathrm{~dB}$, the rule which models offset achieves reaches an error rate below $10^{-5}$, while the rule which does not exhibits an error rate higher than $10^{-1}$.

Examples of radio parameters exhibiting frequency offsets on the order of hundredths of a tone spacing are shown in Table 6.1. Frequency offset for actual crystal oscillators is typically a function of the particular carrier frequency at which the oscillator is driven. A tutorial on the specification of offset for actual oscillators is [91]. The tabulated values of are representative of the capabilities of contemporary platforms, such as the Universal Software Radio Peripheral (USRP) [99].

\subsubsection{Conclusion}

A noncoherent detector for the relay in the two-way relay channel using the digital network coding protocol is developed. The detector is capable of compensating for oscillator frequency offsets, achieving significant performance improvement over a detection rule which does not consider offset. The detector produces soft outputs appropriate for use with soft- 


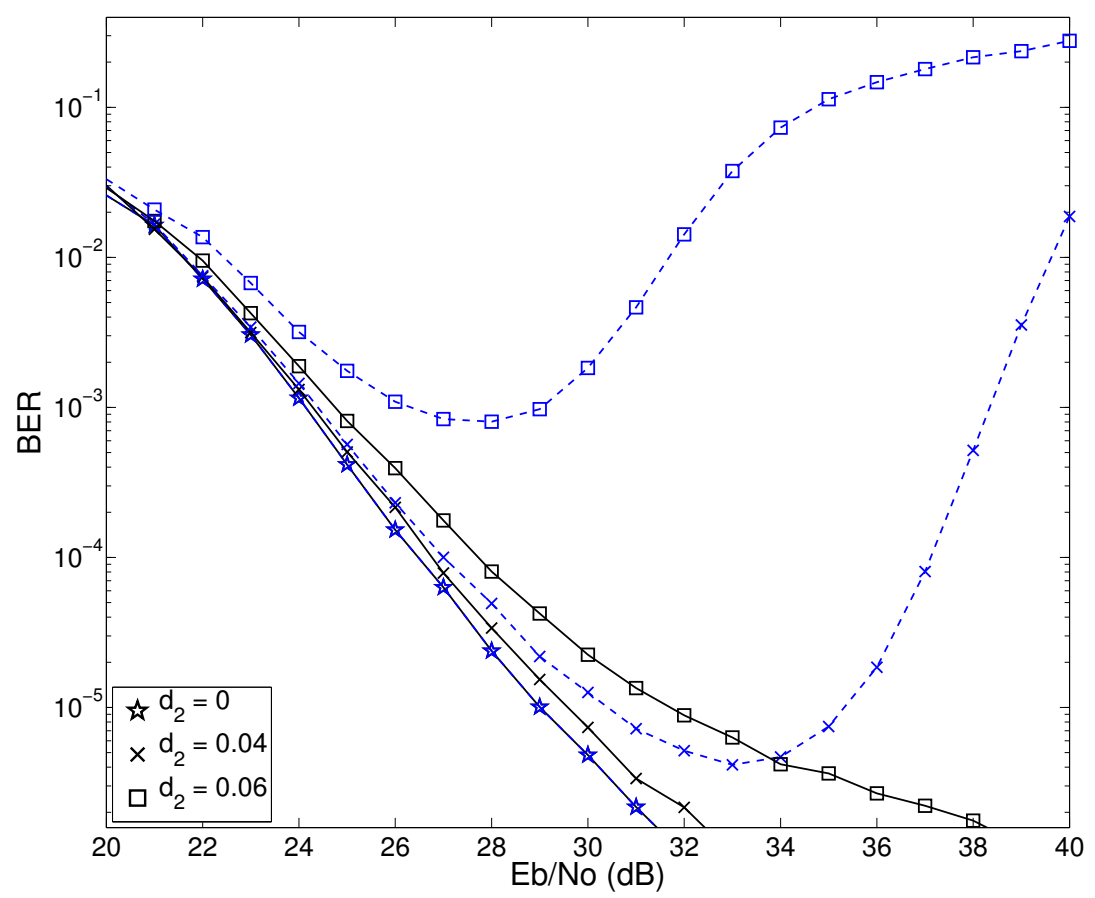

Figure 6.4: Simulated performance of noncoherent detection rule incorporating frequency offset assuming nonzero offsets at both end nodes. Offset $d_{1}=0$ for all cases. A rate 4500/6500 turbo code is applied to all simulations. Blue, dashed lines denote the detection rule which does not model offset, while black, solid lines denote the detection rule which does model offset.

decision decoding algorithms.

When the relay is capable of locking its oscillator frequency to one of the end nodes, the noncoherent detector is capable of achieving uncoded error rates below $10^{-2}$ for frequency offset values at the opposite end node on the order of a few hundredths of a fraction of FSK tone spacing. Appropriate turbo code rates at the end nodes allows the detector which models offset to achieve lower error rates than the detector which does not. As SNR tends to infinity, the error performance of the detector tends to an error floor. Further analysis is required to determine the end node of this floor. When the relay is not capable of locking its oscillator frequency to either end node, performance is dominated by the difference between the offsets at the end nodes. 


\subsection{Reduced Complexity Detection for Network-Coded Slotted ALOHA Using Sphere Decoding}

Throughout the dissertation we have considered PNC in the two-way relay channel containing two end nodes and one relay, where the end nodes act as sources and destinations. In the present section we generalize from two end nodes to an arbitrary number of sources transmitting to the relay simultaneously. Decoding complexity at the relay increases exponentially with the number of sources, and the fundamental challenge is to manage this complexity. We consider a technique that reduces the number of symbols that must be considered for demodulation, and investigate its performance by error-rate simulation.

\subsubsection{Introduction}

Slotted ALOHA (SA) is a multiple-access protocol in which several sources transmit information to a receiver over a timespan denoted as a frame. The frame is divided into several discrete timespans denoted as slots, and sources align their packet transmission times to fall within the slots. The protocol is used in a variety of applications, such as satellite communication.

Recently, several enhancements to classical slotted ALOHA have appeared in the literature. Under the original formulation of SA reception of only one packet per slot is assumed, and reception from two or more sources in a single slot is treated as interference and discarded. All of the enhancements are based on relaxing the assumption of one packet reception per slot and allowing multiple, improving throughput. In [100], each source transmits its packet in multiple slots, deliberately causing interference, and the receiver subtracts noninterfered packets from interfered ones to recover all source packets, a process known as successive interference cancellation (SIC).

A technique has been introduced which improves the throughput of slotted ALOHA using physical-layer network coding (PNC) [5], denoted as network-coded slotted ALOHA (NCSA) [101]. Intuitively, throughput is improved by allowing more than one source to 
transmit a packet during a particular ALOHA slot. For a particular ALOHA frame, the sum of packets in each slot is modeled as a system of linear equations, and the receiver solves the system to recover the packets transmitted by each source. The NCSA algorithm is formulated under the assumption that the detector at the receiver can perform PNC on the packets received in each slot, but does not describe a technique for doing so.

Other works have considered the application of PNC to slotted ALOHA, PNC and sphere decoding, and modulation orders for PNC beyond two. In [102], two schemes are proposed combining PNC and slotted ALOHA, one for terrestrial networks, and one for satellite networks, which require distinct control information. In [103], a lattice-based PNC scheme utilizing compute-and-forward is developed. Higher-order modulations for a system combining PNC and multiuser detection are considered by [104].

The main contribution of the current section is to develop a receiver capable of performing the PNC operation, forming a network-coded packet for each slot. Our work considers a specific modulation, $M$-ary orthogonal modulation. The previous chapters of this dissertation have treated the development of PNC receivers for the two-way relay channel in detail, considering two users. The current work extends the previous, considering an arbitrary number of users.

Detecting the network-coded packet for a particular slot requires performing detection on the sum of symbols transmitted by each source. The symbol determined by performing detection on this sum constellation is denoted as the super-symbol. In a fading environment where the channels between each source and the destination have independent fading coefficients, the number of possible super symbols is exponential in the number of sources.

Maximum likelihood (ML) detection implemented by comparing all possible super symbols to the received symbol clearly becomes intractable for increasing modulation order and number of sources. This motivates the development of a detection scheme with reduced complexity. A well-known detection technique having complexity which is independent of the constellation size is sphere decoding [105]. This work develops a sphere decoder which detects the super-symbols comprising the network-coded packets in an ALOHA slot. Detecting the 


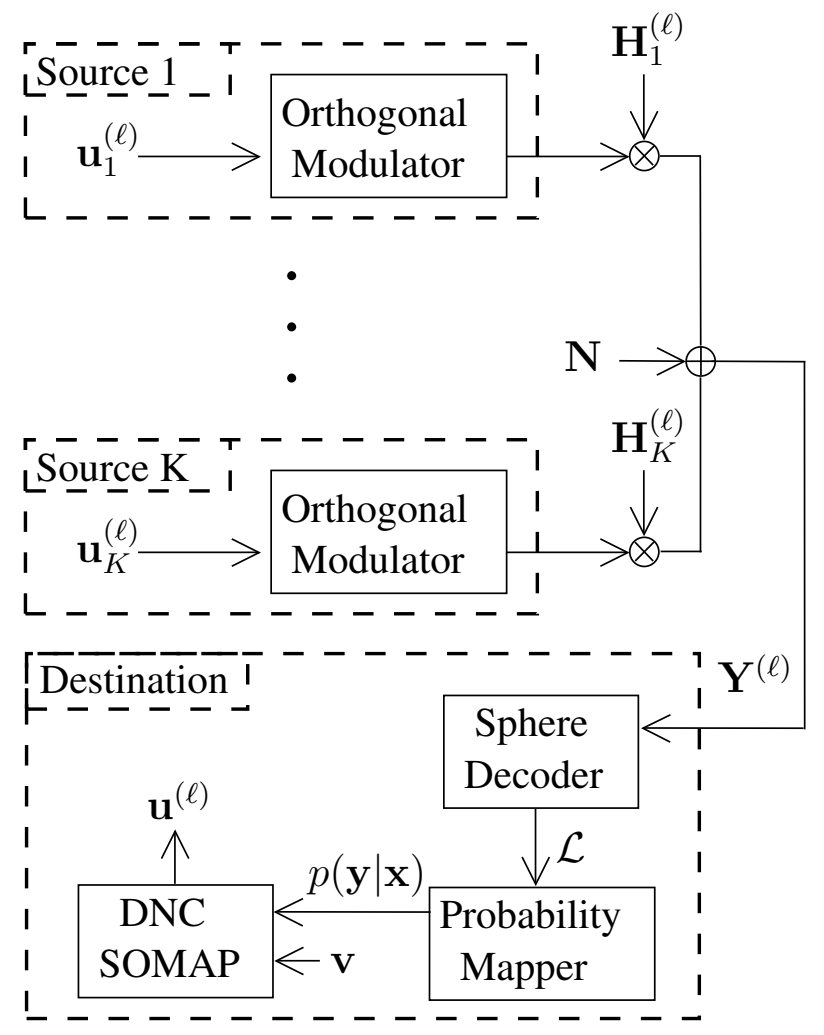

Figure 6.5: System Model

transmitted super symbol enables recovery of the bits in the network-coded packet.

The rest of the this work is presented as follows. Section 6.2.2 describes the system model. Section 6.2.3 develops the sphere decoder for physical-layer network coding. Section 6.2.4 presents simulation results, and Section 6.2.5 provides concluding remarks.

\subsubsection{System Model}

In this section, the modeling assumptions used throughout the work are developed. The modulation scheme, channel model, and source and destination architectures are presented.

\section{Transmission by Sources}

The end nodes $\mathcal{N}_{k}, k \in\{1,2, \ldots, K\}$ generate length- $L$ binary information sequences $\mathbf{u}_{k}=\left[u_{k, 1}, \ldots, u_{k, L}\right]$. Let $\mathcal{D}=\{0, \ldots, M-1\}$ denote the set of integer indices corresponding 
to each orthogonal dimension, where $M$ is the modulation order. The number of bits per symbol is $\mu=\log _{2} M$. The information sequences $\mathbf{u}_{k}$ from each source are divided into $N_{q}=L / \mu$ sets of bits, which are passed to an $M$-ary orthogonal modulator. The modulator maps each set of bits to an $M$-ary symbol $q_{k, i} \in \mathcal{D}$, where $k$ denotes the source, and $i$ denotes the $i$-th symbol, constructing a frame.

The symbols are represented in discrete time by the set of column vectors $\mathbf{x}_{k, i}$. The column vector $\mathbf{x}_{k, i}$ is length $M$, contains a 1 at vector position $q_{k, i}$, and 0 elsewhere. The modulated frame from source $\mathcal{N}_{k}$ is represented by the matrix of symbols $\mathbf{X}_{k}=\left[\mathbf{x}_{k, 1}, \ldots, \mathbf{x}_{k, N_{q}}\right]$, having dimensionality $M \times N_{q}$.

\section{Channel Model}

All of the channels in the system are modeled as block-fading channels. A block is defined as a set of $N$ symbols that all experience the same fading coefficient. The duration of each block corresponds roughly to the channel coherence time. The frame $\mathbf{X}_{k}$ transmitted by source $\mathcal{N}_{k}$ may be partitioned into $N_{b}=N_{q} / N$ blocks according to

$$
\mathbf{X}_{k}=\left[\begin{array}{lll}
\mathbf{X}_{k}^{(1)} & \ldots & \mathbf{X}_{k}^{\left(N_{b}\right)}
\end{array}\right]
$$

where each block $\mathbf{X}_{k}^{(\ell)}, 1 \leq \ell \leq N_{b}$, is an $M \times N$ matrix, and the values of $N_{q}$ and $N$ are chosen such that $N_{b}$ is an integer. The vector of information bits mapped to the symbols in this block is denoted as $\mathbf{u}_{k}^{(\ell)}$, containing $N \mu$ bits. The channel associated with block $\mathbf{X}_{k}^{(\ell)}$ is represented by the $M \times M$ diagonal matrix $\mathbf{H}_{k}^{(\ell)}=\operatorname{diag}\left(h_{k}^{(\ell)}\right)$, where $h_{k}^{(\ell)}=\alpha_{k}^{(\ell)} e^{j \theta_{k}^{(\ell)}}, \alpha_{k}^{(\ell)}$ is a real-valued fading amplitude distributed Rayleigh $(1 / \sqrt{2})$ and $\theta_{k}^{(\ell)}$ is a random phase shift distributed $U(0,2 \pi]$. It is assumed that the frames transmitted by the sources are received in perfect synchronization at the destination. The $\ell^{t h}$ block at the output of the relay receiver's matched-filters is then

$$
\mathbf{Y}^{(\ell)}=\sum_{k=1}^{K} \mathbf{H}_{k}^{(\ell)} \mathbf{X}_{k}^{(\ell)}+\mathbf{N}^{(\ell)}
$$

where $\mathbf{N}^{(\ell)}$ is an $M \times N$ noise matrix having elements which are i.i.d. circularly-symmetric complex Gaussian random variates. 


\section{Destination Reception}

The goal of the destination receiver is to detect the network-coded sum of bits transmitted by the sources

$$
\mathbf{u}=\sum_{k=1}^{K} \mathbf{u}_{k}
$$

where the sum is taken modulo-2. The elements of vector $\mathbf{u}$ are then

$$
u_{i}=u_{1, i} \oplus u_{2, i} \oplus \ldots \oplus u_{K, i} \quad i \in\{1, \ldots, L\}
$$

where $\oplus$ is the exclusive-or operation. The network-coded bits experiencing the $\ell$-th fading block are denoted as $\mathbf{u}^{(\ell)}$, containing $N \mu$ bits.

Consider a single symbol transmission period. The constellation formed by the sum of all possible combinations of symbols which can be transmitted by the sources is denoted as the super-symbol constellation and is defined as

$$
\mathbf{x}=\sum_{k=1}^{K} h_{k} \mathbf{x}_{k} .
$$

Under orthogonal modulation, the cardinality of this constellation is $M^{K}$. The networkcoded bits represented by each super-symbol are defined as

$$
b_{i}(\mathbf{x})=b_{i}\left(\mathbf{x}_{1}\right) \oplus b_{i}\left(\mathbf{x}_{2}\right) \oplus \ldots \oplus b_{i}\left(\mathbf{x}_{K}\right)
$$

where the mapping $b_{i}(\cdot)$ selects the $i$-th bit mapped to the symbol, $b_{i}(\mathbf{x})=u_{i}$, and $b_{i}\left(\mathbf{x}_{k}\right)=$ $u_{k, i}$.

The receiver performs detection on a frame of channel observations $\mathbf{Y}$ one observation at a time. A single observation is represented by a single column of $\mathbf{Y}$ and is denoted as $\mathbf{y}$. The operations performed on every channel observation are the same, thus, dependence on a particular symbol interval is dropped. A channel observation is comprised of the sum of symbols $q_{k}$ transmitted by the sources during a single symbol interval.

A conventional demodulator performs detection by computing the conditional probability of receiving every possible super-symbol $p(\mathbf{y} \mid \mathbf{x})$, an exhaustive computation which grows 
exponentially in the number of sources [30]. Sphere decoding reduces the number of required computations by determining a list $\mathcal{L}$ of $N_{S}$ candidate super-symbols which fall within a specified radius from the received channel observation. Details of the sphere decoder are provided in Section 6.2.3.

The symbol probabilities are transformed to the set of $\mu$ log-likelihood ratios (LLRs) associated with each network-coded bit mapped to a particular super-symbol. Denote this operation as digital network-coded soft mapping (DNC-SOMAP) [30]. To detect the networkcoded bits, a hard decision is made on each LLR. The LLR $z_{i}$ of the $i$-th network-coded bit mapped to the super-symbol is computed as

$$
z_{i}=\max _{\mathbf{x}: u_{i}=1} *\left[\log p(\mathbf{y} \mid \mathbf{x})+\sum_{\substack{j=0 \\ j \neq i}}^{\mu-1} u_{j} v_{j}\right]-\max _{\mathbf{x}: u_{i}=0}\left[\log p(\mathbf{y} \mid \mathbf{x})+\sum_{\substack{j=0 \\ j \neq i}}^{\mu-1} u_{j} v_{j}\right] .
$$

where $p(\mathbf{y} \mid \mathbf{x}) \in \mathcal{L}, u_{i}$ and $u_{j}$ are the $i$-th and $j$-th network-coded bits mapped to supersymbol $\mathbf{x}$, and $v_{j}$ is the a-priori LLR of network-coded bit $u_{j}$ which may be fed back from soft-output channel decoder. This work considers hard-decision decoding, so all $v_{j}=0$. The max-star operator is defined as

$$
\max _{i} *\left\{x_{i}\right\}=\log \left\{\sum_{i} e^{x_{i}}\right\}
$$

where the binary max-star operator is $\max *(x, y)=\max (x, y)+\log \left(1+e^{-|x-y|}\right)$ and multiple arguments imply a recursive relationship; for example: $\max *(x, y, z)=\max *(x, \max *(y, z))$.

The value taken by the pdf $p(\mathbf{y} \mid \mathbf{x})$ is dependent on the available channel state information. In this work it is assumed that the destination has perfect knowledge of the channel gains and noise variance

$$
p(\mathbf{y} \mid \mathbf{x})=\left(\frac{1}{\pi N_{0}}\right)^{M} \exp \left\{-\frac{1}{N_{0}}\|\mathbf{y}-\mathbf{x}\|^{2}\right\}
$$

\subsubsection{List Sphere Decoder}

The goal of the list sphere decoder (LSD) [105] is to determine the set of super-symbols $\mathcal{L}$ which fall within a specified distance $r$ from the channel observation. Specifically, the LSD 
searches for super-symbols satisfying the following relation

$$
(\mathbf{x}-\mathbf{y})^{*}(\mathbf{x}-\mathbf{y}) \leq r^{2}
$$

where $(\cdot)^{*}$ denotes the conjugate transpose operation, and $r$ is the radius from the received channel observation $\mathbf{y}$. Knowledge of the fading coefficients between each source and the destination is used to construct the set of constellation points which fall within the specified radius.

The efficiency of the sphere decoder algorithm is based on recursively computing Eq. (6.36) as the summation

$$
\sum_{k=1}^{M}\left|x_{k}-y_{k}+\sum_{j=k+1}^{M} x_{j}-y_{j}\right|^{2} \leq r^{2}
$$

where the subscripts $k$ and $j$ denote the $k$-th and $j$-th components of vectors $\mathbf{x}$ and $\mathbf{y}$. The term $k=M$ yields

$$
\left|x_{M}-y_{M}\right| \leq r
$$

To efficiently compute whether a super-symbol in the received constellation falls within the specified radius $r$ from the channel observation, a parametric description of the constellation is required. Recall that the modulation scheme is modeled using an $M$-dimensional complex vector. Since each vector component is complex valued, each may be regarded as a twodimensional vector space having in-phase and quadrature components. The LSD recursively detects the set of in-phase and quadrature components within each vector component which fall within $r$ to form the list of received points.

Note that the $m$-th component of $\mathbf{x}$ takes the following value in the absence of noise

$$
x_{m}=\sum_{k=1}^{K} h_{k} x_{k, m}
$$

where $x_{k, m}$ is the $m$-th component of the symbol transmitted by source $\mathcal{N}_{k}$. Recall that $x_{k, m}$ takes value 1 if the $k$-th source transmitted in the $m$-th dimension and 0 otherwise. It 
follows that $x_{m}$ will take one of $2^{K}$ values during a single symbol period, drawn from the sums of all possible subsets of $\mathbf{h}=\left\{h_{1}, h_{2}, \ldots, h_{K}\right\}$.

Consider the $m$-th vector component formed from the sum of a particular subset of $\mathbf{h}$. The component may be expressed as $x_{m}^{(\ell)}=d_{\ell} e^{i \theta_{\ell}}$, where $\ell$ denotes a subset of $\mathbf{h}$. Denote the $m$-component of $\mathbf{y}$ as $y_{m}=\hat{d}_{m} e^{i \hat{\theta}_{m}}$. Then Eq. (6.38) may be expressed as

$$
\left|x_{M}^{(\ell)}-y_{M}\right|^{2}=d_{\ell}^{2}+\hat{d}_{M}^{2}-2 d_{\ell} \hat{d}_{M} \cos \left(\theta_{\ell}-\hat{\theta}_{M}\right) \leq r^{2}
$$

rearranging

$$
\cos \left(\theta_{\ell}-\hat{\theta}_{M}\right) \geq \frac{d_{\ell}^{2}+\hat{d}_{M}^{2}-r^{2}}{2 d_{\ell} \hat{d}_{M}}
$$

Define the right-hand side of Eq. (6.41) as

$$
\eta=\frac{d_{\ell}^{2}+\hat{d}_{M}^{2}-r^{2}}{2 d_{\ell} \hat{d}_{M}}
$$

The value of $\eta$ determines whether constellation component $x_{M}^{(\ell)}$ falls within the radius as

$$
\begin{cases}\eta>1 & \text { Not within radius } \\ -1 \leq \eta \leq 1 & \text { If } \hat{\theta}_{M}-\cos ^{-1} \eta \leq \theta_{\ell} \leq \hat{\theta}_{M}+\cos ^{-1} \eta \\ \eta<-1 & \text { Within radius. }\end{cases}
$$

Thus, the super-symbol is not within the radius when $\eta$ is greater than 1 , always within the radius when $\eta$ is less than -1 , and conditionally within the radius when $\eta$ falls between -1 and 1. An example is shown in Fig. 6.6.

For terms $k<M$ Eq. (6.37) becomes

$$
\left|x_{k}^{(\ell)}-\left(y_{k}-a_{j}\right)\right| \leq \sqrt{r^{2}-b_{j}}
$$

where the terms $a_{j}$ and $b_{j}$ are

$$
\begin{aligned}
a_{j} & =\sum_{j=k+1}^{M} x_{j}^{(\ell)}-y_{j} \\
b_{j} & =\sum_{j=k+1}^{M}\left|x_{j}^{(\ell)}-y_{j}+\sum_{o=j+1}^{M} x_{o}^{(\ell)}-y_{m}\right|^{2}
\end{aligned}
$$




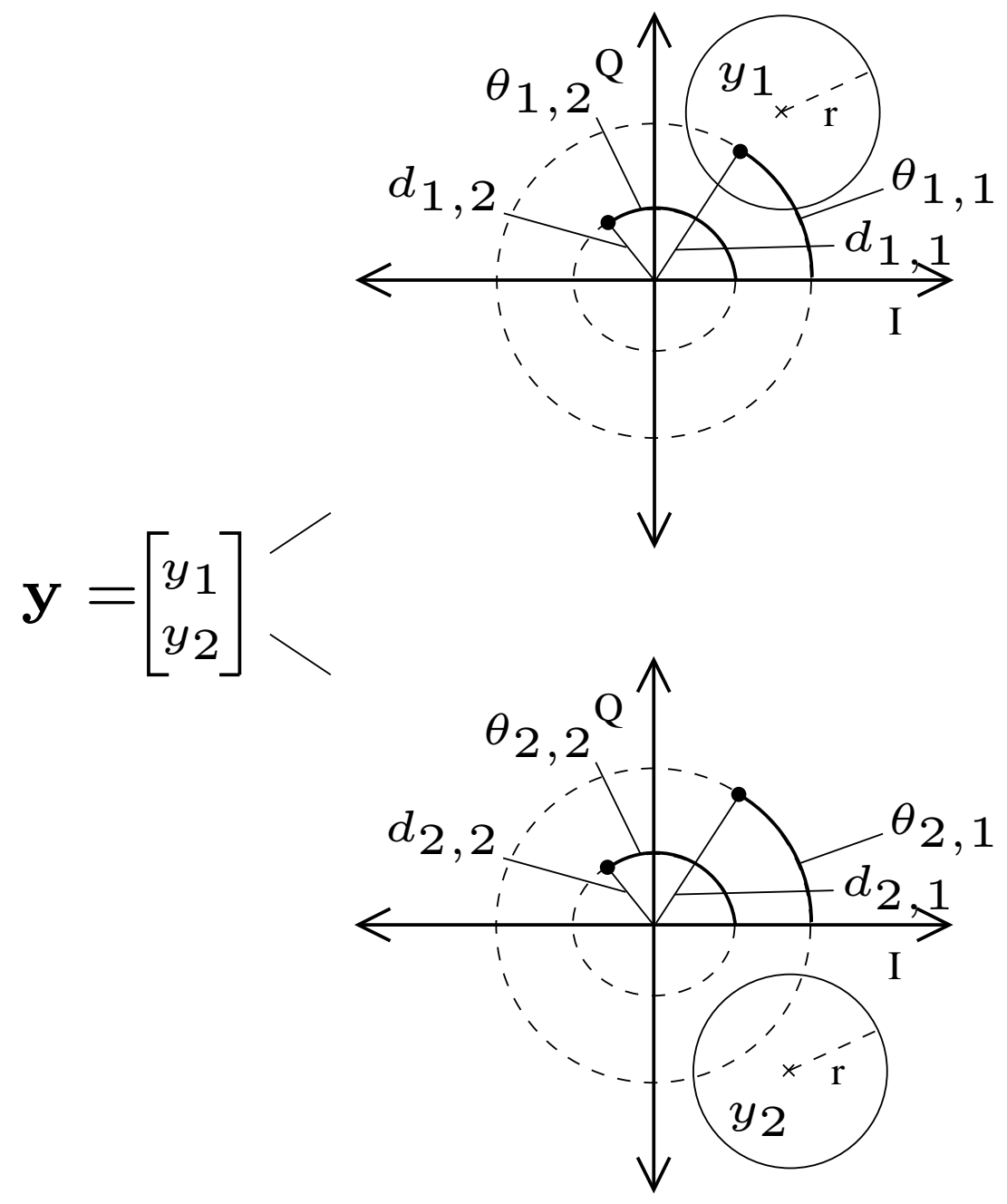

Figure 6.6: Sphere Decoding Example: $M=2$

where $x_{j}^{(\ell)}$ is the constellation component selected at the previous $j$-th dimension. The terms $a_{j}$ and $b_{j}$ can be efficiently accumulated during computation of every $k$-th term of (6.37).

The algorithm proceeds as follows. Beginning with $k=M$, the constellation components $x_{k}^{(\ell)}$ which fall with the radius $r$ are computed for all $\ell$, and one is selected. The value of $k$ is decremented by 1 , constellation components are computed for the new $k$, and a single component is selected once again. If a $k$ value is reached where no points are found, the algorithm backtracks by adding 1 to $k$ and selecting the next available constellation component. 
Once $k=1$ is reached, a super-symbol is selected and its distance from the received point is computed. If the list is not full, the point is added to the list. If the list is full, the distance for the new point is compared to the longest in the list. If the new super-symbol distance is shorter than the last in the list, the new super-symbol is added to the list, replacing the previous longest point.

The algorithm terminates when distances are computed for all super-symbols falling within the radius.

\subsubsection{Simulation Results}

This section presents simulation results. The system described in Section 6.2.2 is simulated via the Monte Carlo method for several values of modulation order and number of sources. All signal-to-noise ratio values are expressed in terms of energy-per-bit $\left(E_{b} / N_{0}\right)$.

The sources are simulated using the following parameters. The number of sources considered is $K=\{2,3,4,5\}$. The information sequence length is $L=2304$. The orthogonal modulation orders are $M=\{2,4\}$, having $\mu=\{1,2\}$ bits per symbol, respectively. The number of symbols per frame is $N_{q}=2304$ under $M=2$ and $N_{q}=1152$ under $M=4$. During a single frame transmission, a uniformly random information sequence $\mathbf{u}_{k}$ is generated for each source and modulated to produce frames $\mathbf{X}_{k}$.

The frames are passed through the channel and corrupted. The block size considered throughout is $N=2304$, which was selected to reduce the required simulation time, as every block requires exhaustively computing the values of the fading coefficient described by Eq. (6.39). The frames are corrupted by the fading coefficients, added, and corrupted by noise according to Eq. (6.28).

For every symbol period in the frame, the sphere decoding algorithm is applied to limit the number of super-symbols required for computation by the DNC-SOMAP. The size of the candidate list $\mathcal{L}$ is set to $N_{S}=5$. The sphere radius is set in proportion to the noise variance $r=2 B N_{0}$, where $B$ is a constant. It was discovered through experimentation that $B=2$ enabled successful decoding in all cases. The sphere decoding algorithm described in 
Section 6.2.3 is applied to all symbol periods in the received frame, and the value $p(\mathbf{y} \mid \mathbf{x})$ is computed for every candidate super-symbol in all $\mathcal{L}$ according to Eq. (6.35).

The values of $p(\mathbf{y} \mid \mathbf{x})$ are passed to the DNC-SOMAP to compute the LLRs of the networkcoded bits for the frame using the relationship described by Eq. (6.33). The network-coded bits comprising a single frame $\mathbf{u}$ are computed by making a hard decision on the LLRs. The a-priori LLRs to the DNC-SOMAP $v_{j}$ are set to 0 for all simulations.

Figs. 6.7 and 6.8 show error-rate performance for modulation orders $M=2$ and $M=4$, respectively. Considering $M=2$, performance degrades by approximately $1 \mathrm{~dB}$ for every source added to the system at error rate $10^{-3}$. Case $M=4$ appears to exhibit similar behavior, as the difference in performance between the best and worst performing curves are similar to the $M=2$ case. The curves in the $M=4$ case are not as smooth as $M=2$, suggesting that more Monte Carlo trials are required to reduce variance.

\subsubsection{Conclusion}

This work has developed and demonstrated a sphere decoder formulation for detecting the network-coded combination of bits transmitted by an arbitrary number of sources. The system has been developed assuming orthogonal modulation and a block fading channel. Simulation results demonstrate that the error-rate performance of the system degrades in proportion to the number of sources.

Several open issues remain. Sphere decoding in the considered system requires calculating all possible combinations of fading coefficients, which requires $2^{K}$ computations. A more efficient approach is necessary for the sphere decoder formulation to be practically efficient. The value of the sphere decoding radius was set in proportion to the channel noise variance, however, more efficiency may be obtained by reducing the radius size. 


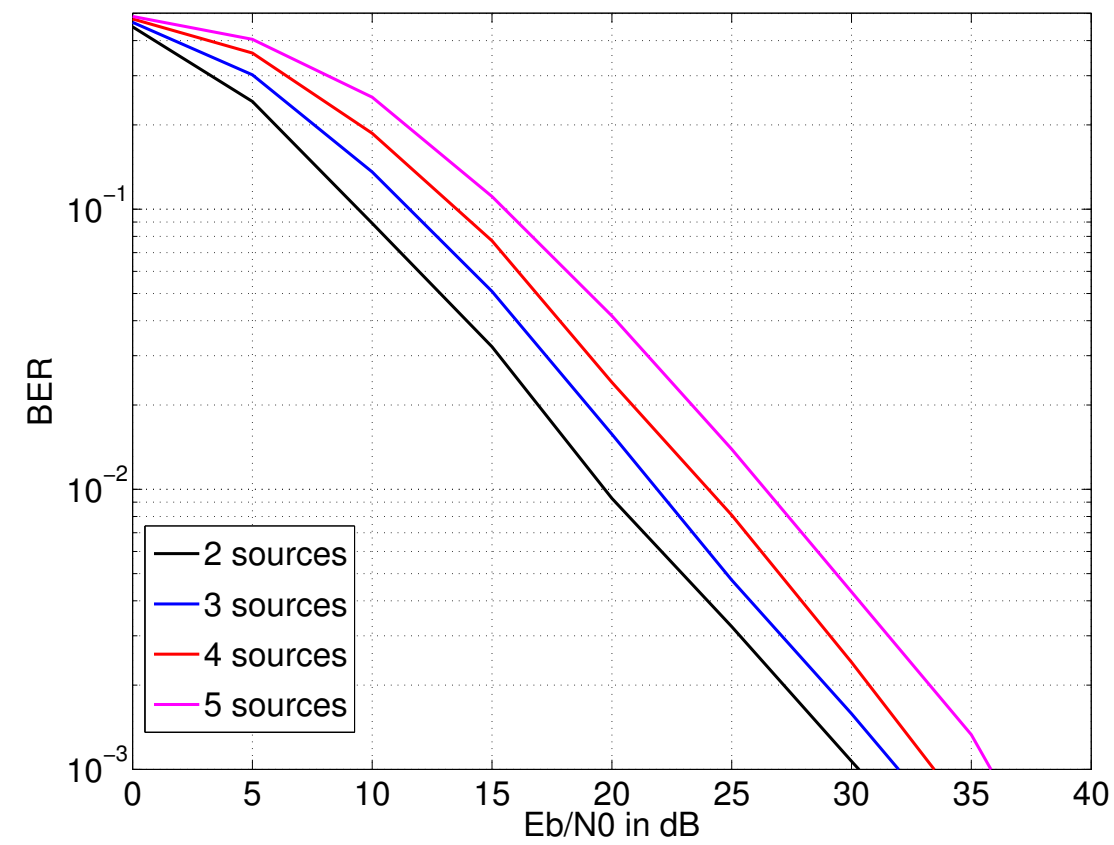

Figure 6.7: Simulated error-rate performance for modulation order $M=2$. The number of sources considered is $K=\{2,3,4,5\}$. The information sequence length is $L=2304$. List sphere decoding uses $N_{S}=5$ symbols per list. A sphere decoding radius $r=4 N_{0}$ is utilized.

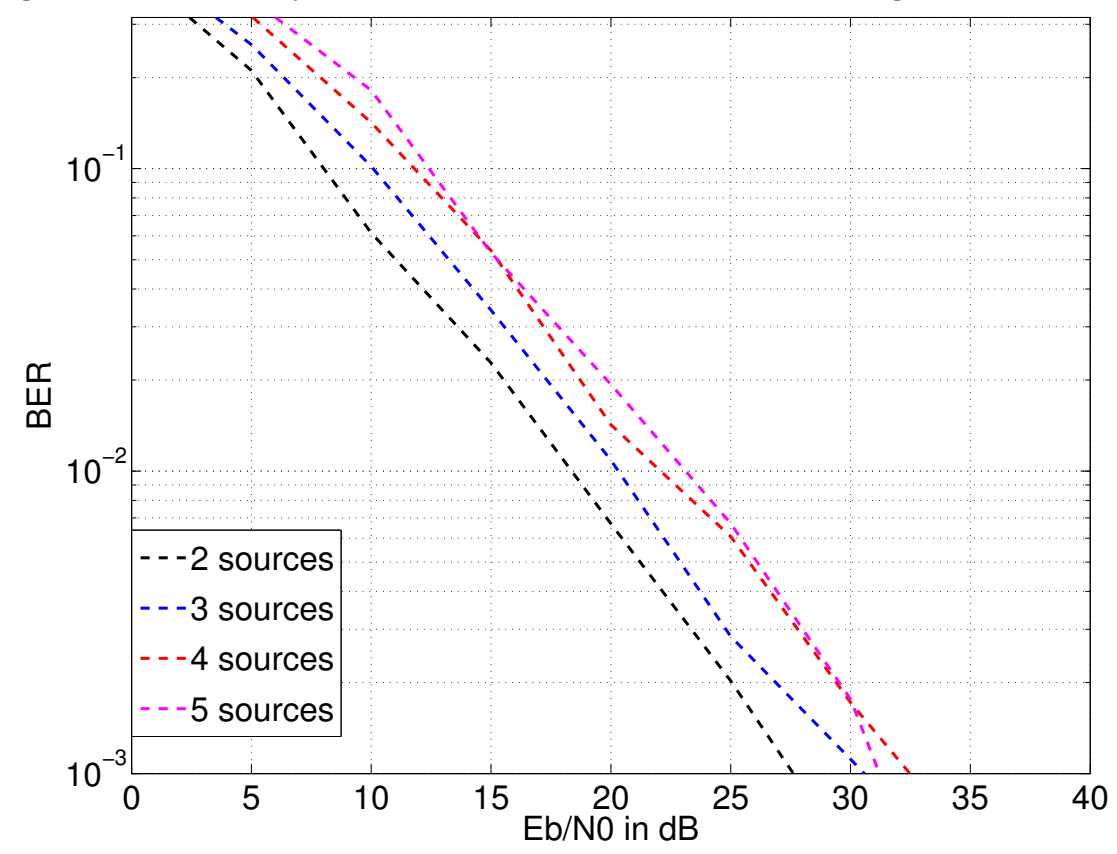

Figure 6.8: Simulated error-rate performance for modulation order $M=4$. See the Fig 6.7 caption or Section 6.2.4 for simulation parameters. 


\section{Chapter 7}

\section{Future Work}

This chapter presents future work, motivated by challenges encountered in the previously described contributions, relaxing previous assumptions, and exploring new techniques. Specifically, Section 7.1 describes an approach for optimizing bandwidth usage, 7.2 proposes analytical performance bounds, 7.3 describes a novel LDPC code construction technique, and 7.4 raises the challenge of analyzing performance limits for our analog network coding demodulator formulation.

\subsection{Non-Orthogonal FSK with Bandwidth Constraint}

Throughout the dissertation we have assumed that the frequency spacing between FSK tones is selected so that the tones are orthogonal. While orthogonality simplifies demodulator design by eliminating inter-tone correlation, bandwidth efficiency can be improved by reducing the tone spacing, deliberately introducing correlation. Inter-tone correlation necessitates extending the demodulator formulations developed in this dissertation. The spacing between tones is parameterized by the modulation index, which is a positive, continuous parameter proportional to the tone spacing. In general, the bandwidth usage of coded FSK is parameterized by the modulation index, channel code rate, and modulation order. When a 
bandwidth constraint is applied to the physical-layer network-coded two-way relay channel using FSK, there will be a particular combination of parameters that minimize the transmission energy required to achieve an arbitrarily low bit error rate. Information-theoretic analysis to optimize these parameters has been applied in a single source-destination system [106] [?]. Physical-layer network coding gives rise to new considerations for bandwidth optimization, namely, the end nodes can use different modulation indices and occupy different portions of spectrum.

\subsection{Analytical Performance Bounds}

The performance analysis in this dissertation is based on Monte Carlo simulation of error rate and capacity. An analytical approach to performance analysis provides an alternative approach that is efficient for some cases, such as determining uncoded error-rate performance. For example, consider computing the symbol error rate during the digital network-coded multiple-access stage using $M$-FSK. The received constellation at the relay grows quickly with modulation order, containing $M^{2}$ constellation points. In addition, the received constellation is located in a $2 M$-dimensional space. The dense received constellation and high dimensionality may make an exact calculation of the symbol error rate challenging. Additionally, analyzing performance in a fading channel or in AWGN with phase-fading requires marginalizing over the appropriate probability distributions. These challenges motivate the use of approximation techniques. In [64], a union bound approximation for the symbol error rate is derived for the multiple-access stage considering non-binary network-coded symbols and two-dimensional channel symbols. A similar approach could be used for the system considered in this dissertation to approximate the symbol error rate.

\subsection{Channel Code Construction}

The LDPC code optimization technique applied in this dissertation is based on identifying degree distributions exhibiting desirable information transfer characteristics. The generated 
LDPC codes are variable-irregular, meaning that the columns of the parity check matrix contain varying numbers of 1's. While irregular codes exhibit good error rate performance in the waterfall region, they exhibit an error floor at low error rates. Regular LDPC codes, which have constant row and column weight, do not exhibit error floors, but do not perform as well in the waterfall region.

Recently, intense research effort has been paid to a class of LDPC codes having parity check matrices with a band-like structure similar to the generator matrices of convolutional codes, constructed using interconnected graphical structures referred to as protographs. Due to the interconnecting graph construction method, these codes are referred to as spatially coupled LDPC (SC-LDPC) codes [107]. SC-LDPC codes exhibit properties suggesting that good performance may be obtained in the waterfall region without error floors. It is not yet known whether good constructions exist for the PNC two-way relay channel.

A channel coding technique for the binary-input discrete memoryless (BDMC) channel has recently been introduced that exhibits capacity-approaching performance using a novel construction technique [108]. The technique is based on the observation that given $N$ independent $\mathrm{BDMC}$ channels, it is possible to synthesize a subset of these channels such that the capacity of the subsets tends to 1 through polarization. Polarization is an operation where a certain subset of $N$ bits is repeated more often than others in order to achieve an arbitrarily low error rate for the repeated bits. Some benefits of polar coding are provable bounds on performance and bounded complexity of encoding and decoding. The performance of these codes in the noncoherent physical-layer network-coded two-way relay channel is not known.

It has been recognized that channel coding performance in the multiple-access stage can be improved by considering quaternary channel codes [64]. The primary reason that quaternary codes can outperform binary is that the exclusive-or operation reduces the number of possible values of each bit from four to two, i.e. $b=b_{1} \oplus b_{2}$ collapses four possible inputs to only two possible outputs. One tradeoff to improved performance is increased decoding complexity, as decoding is performed over a quaternary symbol set rather than binary. Simulation results considering anti-gray-mapped QPSK over an ergodic Rayleigh fading channel 
using a rate $1 / 2$ convolutional code indicate that quaternary decoding can outperform binary by as much as $4 \mathrm{~dB}$.

\subsection{Improved Analog Network Coding}

In our investigation of analog network coding, it was discovered that uncoded error rate simulations exhibited a sharp failure in performance after reaching particular signal-to-noise ratios. The exact SNR value where failure occurs is a function of the number of terms computed in the demodulator infinite series. An analysis of the demodulator formulation is required to determine the reasons for failure, and if the failure is due to an inherent property of the analysis, or an artifact of simulation. If failure occurs due to the demodulator formulation, it is desirable to find another.

There are a number of potential contributions for ANC that were made for DNC, and some that are ANC specific. In this work we have not considered capacity analysis and code design for ANC. Additionally, the complicated form of the ANC end node demodulator motivates the investigation of a partially coherent formulation where noncoherent operation is assumed in the MA stage and coherent is assumed in the BC stage. The coherence assumption can be justified by noting that in the broadcast phase, only the relay is transmitting, so the end nodes receivers must only by synchronized to one oscillator, and only one node is transmitting, so MA interference occurs. In circumstances where phase corruption is unknown but constant for a block of symbols, a multisymbol decoder could be formulated for improved performance over a symbol-by-symbol decoder [109]. 


\section{References}

[1] E. Dahlman, S. Parkvall, and J. Skold, 4G: LTE/LTE-Advanced for Mobile Broadband, Academic Press, 1st edition, 2011.

[2] T. M. Cover and J. A. Thomas, Elements of Information Theory, Wiley-Interscience, 2006.

[3] V. Tarokh et. al., New Directions in Wireless Communications Research, SpringerVerlag US, 1st edition, 2009.

[4] C. Hausl and J. Hagenauer, "Iterative network and channel decoding for the two-way relay channel," Proc. IEEE Int. Conf. on Commun., vol. 4, pp. 1568-1573, June 2006.

[5] S. Zhang, S. C. Liew, and P. P. Lam, "Hot topic: Physical-layer network coding," in Proc. ACM Annual Int. Conf. on Mobile Computing and Networking, Sept. 2006, pp. 358-365.

[6] S. C. Liew, S. Z., and L. Lu, "Physical-layer network coding: Tutorial, survey, and beyond," Physical Communication, vol. 6, pp. 4-42, 2013.

[7] M. Chen and A. Yener, "Multiuser two-way relaying: Detection and interference management strategies," IEEE Trans. Wireless Commun., vol. 8, no. 8, pp. 42964305, Aug. 2009.

[8] J. Sørensen, R. Krigslund, P. Popovski, T. K. Akino, and T. Larsen, "Scalable denoiseand-forward in bidirectional relay networks," Computer Networks, vol. 54, no. 10, pp. 1607-1614, June 2010.

[9] S. Zhang, S. C. Liew, and L. Lu, "Physical layer network coding schemes over finite and infinite fields," IEEE Global Telecommun. Conf., pp. 1-6, Dec. 2008.

[10] S. Katti, S. Gollakota, and D. Katabi, "Embracing wireless interference: analog network coding," Proc. ACM SIGCOMM, pp. 397-408, 2007.

[11] L. Lu and S. C. Liew, "Asynchronous physical-layer network coding," IEEE Trans. Wireless Commun., vol. 11, no. 2, pp. 819-831, February 2012. 
[12] X. Wu, C. Zhao, and X. You, "Joint LDPC and physical-layer network coding for asynchronous bi-directional relaying," IEEE J. Select. Areas Commun., vol. 31, no. 8, pp. 1446-1454, August 2013.

[13] W. Liang, K. Xu, H. Tian, and Y. Xu, "Joint design of LDPC and physical-layer network coding for bi-directional relay system in the presence of insufficient timing synchronization," Proc. IEEE Int. Conf. Wireless Comm. Sig. Proc., pp. 1-5, Nov 2011.

[14] P. C. Wang, Y. C. Huang, and K. R. Narayanan, "Asynchronous physical-layer network coding with quasi-cyclic codes," IEEE J. Select. Areas Commun., vol. 33, no. 2, pp. 309-322, Feb 2015.

[15] S. S. Ullah, S. C. Liew, L. Lu, and L. You, "Physical-layer network coding: A high performance PHY-layer decoder," Proc. IEEE Int. Conf. on Commun., pp. 1-6, May 2016.

[16] A. C. Marcum, J. V. Krogmeier, D. J. Love, and A. Sprintson, "Analysis and implementation of asynchronous physical layer network coding," IEEE Trans. Wireless Commun., vol. 14, no. 12, pp. 6595-6607, Dec 2015.

[17] D. Torrieri, Principles of Spread-Spectrum Communication Systems, Springer Publishing Company, Inc., 2nd edition, 2011.

[18] J. G. Proakis and M. Salehi, Digital Communications, McGraw-Hill, Inc., New York, NY, fifth edition, 2008.

[19] E.C.Y. Peh, Ying-Chang Liang, and Yong Liang Guan, "Power control for physicallayer network coding in fading environments," in Proc. IEEE Personal Indoor and Mobile Radio Commun. Conf., 2008, pp. 1-5.

[20] S. Katti, H. Rahul, Wenjun Hu, D. Katabi, M. Medard, and J. Crowcroft, "XORs in the air: Practical wireless network coding," IEEE/ACM Trans. Netw., pp. 497-510, June 2008.

[21] P. Popovski and H. Yomo, "Wireless network coding by amplify-and-forward for bidirectional traffic flows," IEEE Commun. Lett., vol. 11, pp. 16-18, Jan. 2007.

[22] J. Sørensen, R. Krigslund, P. Popovski, T. Akino, and T. Larsen, "Physical layer network coding for FSK systems," IEEE Commun. Lett., vol. 13, no. 8, pp. 597-599, Aug. 2009.

[23] M. C. Valenti, D. Torrieri, and T. Ferrett, "Noncoherent physical-layer network coding using binary CPFSK modulation," Proc. IEEE Military Commun. Conf., pp. 1-7, Oct. 2009. 
[24] T. Cui, F. Gao, and C. Tellambura, "Physical layer differential network coding for two-way relay channels," in Proc. IEEE Global Telecommun. Conf. (Globecom), Dec. 2008.

[25] K. Zhu and A. G. Burr, "A simple non-coherent physical-layer network coding for transmissions over two-way relay channels," Proc. IEEE Global Commun. Conf., pp. 2268-2273, Dec. 2012.

[26] D.-Y. Zhang, Q.-Y. Yu, W.-X. Meng, and C. Li, "2FSK modulation for multiuser physical-layer network coding network," Proc. IEEE Int. Conf. on Commun., pp. 514-519, June 2014.

[27] X. Dang, Z. Liu, B. Li, and X. Yu, "Noncoherent multiple-symbol detector of binary CPFSK in physical-layer network coding," IEEE Commun. Lett., vol. 20, no. 1, pp. 81-84, Jan. 2016.

[28] Q.-Y. Yu, D.-Y. Zhang, H.-H. Chen, and W.-X. Meng, "Physical-layer network coding systems with MFSK modulation," IEEE Trans. Veh. Technol., vol. 65, no. 1, pp. 204-213, Jan. 2016.

[29] T. Ferrett, M. C. Valenti, and D. Torrieri, "Noncoherent digital network coding using multi-tone CPFSK modulation," Proc. IEEE Military Commun. Conf., pp. 299-304, Nov. 2011.

[30] T. Ferrett, M. C. Valenti, and D. Torrieri, "An iterative noncoherent relay receiver for the two-way relay channel," Proc. IEEE Int. Conf. on Commun., pp. 5903 - 5908, June 2013.

[31] T. Ferrett and M. C. Valenti, "LDPC code design for noncoherent physical layer network coding," Proc. IEEE Int. Conf. on Commun., pp. 2054-2059, June 2015.

[32] C. Shannon, "A mathematical theory of communication," Bell System Technical Journal, vol. 27, pp. 379-423, 623-656, July, October 1948.

[33] S. Lin and D. J. Costello, Error Control Coding, Prentice Hall, Inc., Upper Saddle River, NJ, second edition, 2004.

[34] G. Caire, G. Taricco, and E. Biglieri, "Bit-Interleaved Coded Modulation," IEEE Trans. Inform. Theory, vol. 44, no. 3, pp. 927-946, May 1998.

[35] X. Li and J. A. Ritcey, "Bit-interleaved coded modulation with iterative decoding," IEEE Commun. Lett., vol. 1, no. 6, Nov. 1997.

[36] S. Zhang and S. C. Liew, "Channel coding and decoding in a relay system operated with physical-layer network coding," IEEE J. Select. Areas Commun., vol. 27, no. 5, pp. 788-789, June 2009. 
[37] X. Li, S. Zhang, and G. Qian, "Mapping and coding design for channel coded physicallayer network coding," IEEE Int. Workshop on High Mobility Wireless Commun., pp. 120-125, Nov. 2013.

[38] S. ten Brink, G. Kramer, and A. Ashikhmin, "Design of low-density parity-check codes for modulation and detection," IEEE Trans. Commun., vol. 52, no. 4, pp. 670-678, April 2004.

[39] L. Peng and G. Zhu, "Improving performance of some irregular LDPC codes by means of three optimization techniques," Glob. Mobi. Cong., pp. 1-5, June 2009.

[40] J. Li and Z. Lin, "Design of physical layer network coded LDPC code for a multipleaccess relaying system," IEEE Commun. Lett., vol. 17, no. 4, pp. 749-752, April 2013.

[41] R. Asvadi, T. Matsumoto, and M. Juntti, "LDPC code optimization with joint sourcechannel decoding of quantized gauss-markov signals," Proc. IEEE Int. Conf. on Commun., pp. 5233 - 5238, June 2014.

[42] L. Yang, T. Yang, J. Yuan, and J. An, "Achieving the near-capacity of two-way relay channels with modulation-coded physical-layer network coding," IEEE Trans. Wireless Commun., vol. 14, no. 9, pp. 5225-5239, Sept 2015.

[43] P. Chen, K. Su, Y. Fang, and L. Kong, "The design of protograph LDPC codes for channel-coded physical-layer network coding," in Proc. IEEE Personal Indoor and Mobile Radio Commun. Conf., Sept 2016, pp. 1-6.

[44] S. ten Brink, "Convergence of iterative decoding," IEEE Commun. Lett., vol. 35, no. 10, pp. 806-808, May 1999.

[45] T. Ferrett, M. C. Valenti, and D. Torrieri, "Receiver design for noncoherent digital network coding," Proc. IEEE Military Commun. Conf., pp. 2096-2101, Oct. 2010.

[46] M. C. Valenti, D. Torrieri, and T. Ferrett, "Noncoherent physical-layer network coding with FSK modulation: Relay receiver design issues," IEEE Trans. Commun., vol. 59, Sept. 2011.

[47] M. C. Valenti and T. Ferrett, "Noncoherent LDPC-coded physical-layer network coding using multitone FSK," IEEE Trans. Commun., submitted for publication.

[48] T. Ferrett and M. C. Valenti, "Noncoherent analog network coding using LDPC-coded FSK," Proc. IEEE Int. Conf. on Commun., pp. 1-6, May 2017.

[49] T. Ferrett, H. Ochiai, and M. C. Valenti, "Physical-layer network coding using FSK modulation under frequency offset," IEEE Trans. Veh. Technol., pp. 1-5, May 2012. 
[50] T. Ferrett and M. C. Valenti, "Reduced complexity detection for network-coded slotted aloha using sphere decoding," in Asilomar Conf. on Sig., Syst. and Comput., Nov 2015, pp. 1611-1615.

[51] D. Wang, S. Fu, and K. Lu, "Channel coding design to support asynchronous physical layer network coding," in Proc. IEEE Global Telecommun. Conf. (Globecom), Dec. 2009.

[52] A. Zhan, C. He, and L. Jian, "A Turbo-BICM Based Scheme for Joint Network Coding and Channel Coding," Proc. IEEE Int. Conf. on Commun., May 2010.

[53] S. S. Ullah, G. Liva, and S. C. Liew, "Physical-layer network coding: A random coding error exponent perspective," CoRR, vol. abs/1702.01311, 2017.

[54] B. Jiang, F. Gao, X. Gao, and A. Nallanathan, "Channel estimation and training design for two-way relay networks with power allocation," IEEE Trans. Wireless Commun., vol. 9, no. 6, pp. 2022-2032, June 2010.

[55] F. Gao, R. Zhang, and Y. C. Liang, "On channel estimation for amplify-and-forward two-way relay networks," in Proc. IEEE Global Telecommun. Conf. (Globecom), Dec. 2008, pp. 1-5.

[56] X. Li and P. Ho, "A partial coherent detector for orthogonal modulations in two-way relay communications with physical network coding and fading," pp. 630-634, Nov 2014.

[57] X. Li and P. Ho, "A random channel sounding decision feedback receiver for two-way relay communication with pilotless orthogonal signaling and physical-layer network coding," IEEE Trans. Veh. Technol., vol. 65, no. 5, pp. 3086-3099, May 2016.

[58] M. C. Valenti and S. Cheng, "Iterative demodulation and decoding of turbo coded $M$-ary noncoherent orthogonal modulation," IEEE J. Select. Areas Commun., vol. 23, no. 9 , pp. $1738-1747$, Sept. 2005.

[59] "NIST Digital Library of Mathematical Functions," http://dlmf.nist.gov/, Release 1.0.13 of 2016-09-16, F. W. J. Olver, A. B. Olde Daalhuis, D. W. Lozier, B. I. Schneider, R. F. Boisvert, C. W. Clark, B. R. Miller and B. V. Saunders, eds.

[60] J. Hamkins, "An analytic technique to separate cochannel FM signals," IEEE Trans. Commun., vol. 48, pp. 543-546, April 2000.

[61] D. Torrieri, S. Cheng, and M. C. Valenti, "Robust frequency hopping for interference and fading channels," IEEE Trans. Wireless Commun., vol. 56, pp. 1343-1351, Aug. 2008. 
[62] European Telecommunications Standards Institute, "Universal mobile telecommunications system (UMTS): Multiplexing and channel coding (FDD)," 3GPP TS 25.212 version 7.4.0, June 2006.

[63] P. Chen, S. C. Liew, and L. Shi, "Bandwidth-efficient coded modulation schemes for physical-layer network coding with high-order modulations," IEEE Trans. Commun., vol. 65, no. 1, pp. 147-160, Jan. 2017.

[64] H. Yan and H. H. Nguyen, "BICM-ID in two-way relaying communications," Proc. IEEE Int. Conf. on Commun., pp. 1-6, May 2016.

[65] L. Chen, Y. Yakufu, X. Yuan, and Z. Sun, "Design of BICM-ID for two-way relay channels," IEEE Int. Workshop on High Mobility Wireless Commun., pp. 11-15, Oct. 2015.

[66] S. Benedetto, G. Montorsi, D. Divsalar, and F. Pollara, "Soft-input soft-output modules for the construction and distributed iterative decoding of code networks," Eur. Trans. Telecommun., vol. 9, pp. 155-172, Mar.-Apr. 1998.

[67] S. Cheng and M. C. Valenti, "Bit-interleaved turbo-coded noncoherent orthogonal modulation with iterative demodulation and decoding: capacity limits and convergence analysis," Asilomar Conf. on Signals, Syst. and Comput., pp. 2020-2024, Nov. 2004.

[68] M. Noori and M. Ardakani, "On symbol mapping for binary physical-layer network coding with PSK modulation," IEEE Trans. Wireless Commun., vol. 11, no. 1, pp. 21-26, Jan. 2012.

[69] S. Zhang, S. C. Liew, H. Wang, and X. Lin, "Capacity of two-way relay channel," 4th Int. Conf. AccessNets, pp. 219-231, Nov. 2010.

[70] "Digital video broadcasting (DVB)," ETSI EN 302 307 V1.3.1, 2013.

[71] M. C. Valenti and X. Xiang, "Constellation shaping for bit-interleaved LDPC coded APSK," IEEE Trans. Commun., vol. 60, no. 10, pp. 2960-2970, July 2012.

[72] T. Huang, T. Yang, J. Yuan, and I. Land, "Design of irregular repeat-accumulate coded physical-layer network coding for gaussian two-way relay channels," IEEE Trans. Commun., vol. 61, no. 3, March 2013.

[73] A. K. Tanc, T. M. Duman, and C. Tepedelenlioglu, "Design of LDPC codes for twoway relay systems with physical-layer network coding," IEEE Commun. Lett., vol. 17, no. 12, pp. 2356-2359, Dec. 2013.

[74] "Standard for air interface for broadband wireless access systems," IEEE Standard 802.16-12, 2012. 
[75] M. C. Valenti, S. Cheng, and R. Iyer Seshadri, "Turbo and LDPC codes for digital video broadcasting," Turbo Code Applications, p. 301, 2005.

[76] L. Wenwen, C. Yande, X. Kui, T. Hua, and X. Youyun, "Joint LDPC and physical-layer network coding for two-way relay channels with different carrier frequency offsets," Proc. IEEE Int. Conf. Wireless Comm. Sig. Proc., pp. 1-4, 2012.

[77] S. Zhang, S. C. Liew, Q. Zhou, L. Lu, and H. Wang, "Non-memoryless analog network coding in two-way relay channel," Proc. IEEE Int. Conf. on Commun., pp. 1-6, June 2011.

[78] M. H. Azmi, J. Li, J. Yuan, and R. Malaney, "Design of distributed multi-edge type LDPC codes for two-way relay channels," pp. 1-5, June 2011.

[79] M. Yang, Ryan W. E., and Y. Li, "Design of efficiently encodable moderate-length high-rate irregular LDPC codes," IEEE Trans. Commun., vol. 52, no. 4, pp. 564-571, April 2004.

[80] A. Ashikhmin, G. Kramer, and S. Brink, "Extrinsic information transfer functions: Model and erasure channel properties," IEEE Trans. Inform. Theory, vol. 50, no. 11, Nov. 2004.

[81] S. ten Brink, "Convergence behavior of iteratively decoded parallel concatenated codes," IEEE Trans. Commun., vol. 49, no. 10, pp. 1727-1737, Oct. 2001.

[82] D. Torrieri and M. C. Valenti, "Rapidly-converging series representations of a mutualinformation integral," ISRN Commun. and Network., vol. 2011, Article ID 546205, 2011.

[83] I. Maric, A. Goldsmith, and M. Medard, "Analog network coding in the high-SNR regime," IEEE Wireless Netw. Coding Conf., pp. 1-6, June 2010.

[84] C. He and Z. J. Wang, "Closed-form BER analysis of non-coherent FSK in MISO double Rayleigh fading/RFID channel," IEEE Commun. Lett., vol. 15, no. 8, pp. 848-850, Aug. 2011.

[85] H. Lu, Y. Chen, and N. Cao, "Accurate approximation to the PDF of the product of independent Rayleigh random variables," IEEE Antennas and Wireless Propag. Lett., vol. 10, pp. 1019-1022, Sept. 2011.

[86] Y. Yang, W. Chen, O. Li, and L. Hanzo, "Joint rate and power adaptation for amplifyand-forward two-way relaying relying on analog network coding," IEEE Access, vol. 4, pp. 2465-2478, 2016.

[87] L. Song, Y. Li, A. Huang, B. Jiao, and A. V. Vasilakos, "Differential modulation for bidirectional relaying with analog network coding," IEEE Trans. Signal Process., vol. 58, no. 7, pp. 3933-3938, July 2010. 
[88] R. H. Y. Louie, Y. Li, and B. Vucetic, "Practical physical layer network coding for two-way relay channels: Performance analysis and comparison," IEEE Trans. Wireless Commun., vol. 9, no. 2, pp. 764-777, 2010.

[89] N. O'Donoughue and J. M. F. Moura, "On the product of independent complex Gaussians," IEEE Trans. Signal Process., vol. 60, no. 3, pp. 1050-1063, Mar. 2012.

[90] I. S. Gradshteyn and I. M. Ryzhik, Table of Integrals, Series, and Products, Academic Press, Burlington, MA, seventh edition, 2007.

[91] John R. Vig, "Introduction to quartz frequency standards," Electronics and Power Sources Directorate, pp. SLCET-TR-92-1 (Rev. 1), Oct. 1992.

[92] S. Hussain, S.K. Barton, and S.J. Shepherd, "Non-coherent detection of FSK signals in the presence of oscillator phase noise in an AWGN channel.," IEEE Trans. Veh. Technol., pp. 1552-1556, 1994.

[93] Heung-Gyoon Ryu, Yingshan Li, and Jin-Soo Park, "Effects of frequency instability caused by phase noise on the performance of the fast fh communication system," IEEE Trans. Veh. Technol., pp. 1626-1632, Sept. 2004.

[94] H. Wang, W. Zhu, Q. Meng H. Wei, and X. Wan, "The effects of frequency offset on network coding with 4FSK modulation," in Int. Conf. on Adv. Comput. Sci. and Electron. Inform., July 2013, pp. 351-354.

[95] M. Wu, F. Ludwig, M. Woltering, D. Wuebben, A. Dekorsy, and S. Paul, "Analysis and implementation for physical-layer network coding with carrier frequency offset," pp. 1-8, March 2014.

[96] G. Caire and C. Elia, "A New Symbol Timing and Carrier Frequency Offset Estimation Algorithm for Noncoherent Orthogonal M-CPFSK," IEEE Trans. Commun., pp. 13141326, Oct. 1997.

[97] Y. Huang, K. Fan, and C. Huang., "A fully digital noncoherent and coherent GMSK receiver architecture with joint symbol timing error and frequency offset estimation," IEEE Trans. Veh. Technol., pp. 863-874, May 2000.

[98] Yung-Szu Tu and Gregory J. Pottie, "Coherent cooperative transmission from multiple adjacent antennas to a distant stationary antenna through awgn channels," IEEE Trans. Veh. Technol., pp. 130-134, Aug. 2002.

[99] Matt Ettus, "TX and RX daughterboards for the USRP tm software radio system," www.ettus.com, 2011.

[100] E. Paolini, G. Liva, and M. Chiani, "Coded slotted aloha: A graph-based method for uncoordinated multiple access," IEEE Trans. Inform. Theory, vol. 61, no. 12, pp. 6815-6832, Dec. 2015. 
[101] S. Yang, Y. Chen, S. C. Liew, and L. You, "Coding for network-coded slotted aloha," IEEE Inform. Theory Workshop, pp. 1-5, April 2015.

[102] G. Cocco, C. Ibars, D. Gündüz, and O. del Rio Herrero, "Collision resolution in slotted aloha with multi-user physical-layer network coding," IEEE Trans. Veh. Technol., May 2011.

[103] A. Mejri and G. R. B. Othman, "MAP decoder for physical-layer network coding using lattice sphere decoding," in Int. Conf. Telecomun., May 2014, pp. 67-71.

[104] H. Pan, L. Lu, and S. C. Liew, "Network-coded multiple access with higher-order modulations," Proc. IEEE Global Telecommun. Conf. (Globecom), pp. 1-7, Dec. 2015.

[105] B. M. Hochwald and S. ten Brink, "Achieving near-capacity on a multiple-antenna channel," IEEE Trans. Commun., vol. 51, no. 3, pp. 389-399, 2003.

[106] S. Cheng, R. I. Seshadri, M. C. Valenti, and D. Torrieri, "The capacity of noncoherent continuous-phase frequency shift keying," in IEEE Conf. on Inform. Sci. Syst., Mar. 2007, pp. 396-401.

[107] E. Ankan, N. ul Hassan, M. Lentmaier, G. Montorsi, and J. Sayir, "Challenges and some new directions in channel coding," J. Commun. and Netw., vol. 17, no. 4, pp. 328-338, Aug 2015.

[108] E. Arikan, "Channel polarization: A method for constructing capacity-achieving codes for symmetric binary-input memoryless channels," vol. 55, no. 7, pp. 3051-3073, July 2009 .

[109] M. C. Valenti, S. Cheng, and D. Torrieri, "Iterative multisymbol noncoherent reception of coded CPFSK," IEEE Trans. Commun., vol. 58, no. 7, pp. 2046-2054, July 2010. 